\title{
K3 SURFACES WITH INVOLUTION AND ANALYTIC TORSION
}

\author{
KEN-ICHI YOSHIKAWA
}

\section{$\S 1$. Introduction}

In a series of works [Bo3-5], Borcherds developed a theory of modular forms over domains of type IV which admits an infinite product expansion. Such modular forms are said to be Borcherds's product in this paper. Among all Borcherds's products, Borcherds's $\Phi$-function ([Bo4]) has an interesting geometric background; It is a modular form on the moduli space of Enriques surfaces characterizing the discriminant locus. In his construction, $\Phi$-function is obtained as the denominator function of one of the fake monster Lie superalgebras ([Bo2, §14]), although Enriques surface itself plays no role. After Borcherds, Jorgenson-Todorov ([J-T2,3]) and Harvey-Moore $([\mathrm{H}-\mathrm{M}])$ discovered that the Ray-Singer analytic torsion ([R-S]) of an Enriques surface equipped with the normalized Ricci-flat Kähler metric coincides with Borcherds's $\Phi$-function at its period point. The goal of this paper is to give a rigorous proof to their observation and generalize it to an interesting class of $K 3$ surfaces studied by Nikulin ([Ni4]). Let us briefly recall these surfaces.

Let $(X, \iota)$ be a $K 3$ surface with an anti-symplectic involution. Let $M$ be a 2-elementary hyperbolic lattice. The pair $(X, \iota)$ is said to be a 2-elementary $K 3$ surface of type $M$ if the invariant sublattice of $H^{2}(X, \mathbb{Z})$ with respect to the action of $\iota$ is isometric to $M$. Since $X / \iota$ is an Enriques surface when $M \cong \mathrm{II}_{1,9}(2), K 3$ surfaces of this class are a kind of generalizations of Enriques surfaces. (We denote by $\mathrm{I}_{p, q}$ (resp. $\mathrm{II}_{p, q}$ ) the odd (resp. even) unimodular lattice of signature $(p, q)$.) By the Torelli theorem ([P-S-S]) and surjectivity of the period map ([To1]), the moduli space of 2-elementary $K 3$ surfaces of type $M$ is isomorphic to an arithmetic quotient of an open subset of the symmetric bounded domain of type IV via the period map. The period domain is denoted by $\Omega_{M}$, the modular group by $\Gamma_{M}$, and the moduli space by $\mathcal{M}_{M}^{0}=\left(\Omega_{M} \backslash \mathcal{D}_{M}\right) / \Gamma_{M}$. Here, $\mathcal{D}_{M}$ is the discriminant locus. Let $X^{\iota}$ be the fixed locus of $\iota$. Since only one of the irreducible components can have positive genus, $g(M)(\geq 0)$, unless $M \cong \mathrm{II}_{1,9}(2)$ or $\mathrm{II}_{1,1} \oplus E_{8}(-2)$, one can define a map $j_{M}^{0}: \mathcal{M}_{M}^{0} \rightarrow \mathcal{A}_{g(M)}$ to the Siegel modular variety by taking the period point of the Jacobi variety of $X^{\iota}$. (If $M \cong \mathrm{II}_{1,1} \oplus E_{8}(-2), j_{M}$ takes its value in $S^{2}\left(\mathcal{A}_{1}\right)$, the second symmetric product of $\mathcal{A}_{1}$.) Let $\mathcal{F}_{g(M)}$ be the sheaf of Siegel modular forms of weight 1 on $\mathcal{A}_{g(M)}$. We define a $\Gamma_{M}$-invariant sheaf on (an open subset of) $\Omega_{M}$ by $\lambda_{M}:=j_{M}^{*} \mathcal{F}_{g(M)}$. A $\lambda_{M}^{\otimes q}$-valued modular form of weight $p$ (with a character of $\left.\Gamma_{M}\right)$ is said to be an automorphic form of weight $(p, q)$ in this paper whose Petersson norm is denoted by $\|\cdot\|$.

In this paper, we focus on the Ray-Singer analytic torsion regarded as a function on the moduli space of $K 3$ surfaces. Since it coincides with (the Petersson norm 
of) the Jacobi $\Delta$-function for elliptic curves $([\mathrm{R}-\mathrm{S}])$, it is natural to expect that it may yield an interesting modular form for $K 3$ surfaces ([J-T1]). With small modifications, it is the case at least for 2-elementary $K 3$ surfaces. We introduce the following function (see $\S 3$ ) on the polarized period domain of 2-elementary $K 3$ surfaces of type $M$;

$$
\tau_{M}(X, \iota, \kappa):=\operatorname{vol}(X, \kappa)^{\frac{14-r(M)}{8}} \tau(X / \iota, \kappa) \sqrt{\operatorname{vol}\left(X^{\iota},\left.\kappa\right|_{X^{\iota}}\right) \tau\left(X^{\iota},\left.\kappa\right|_{X^{\iota}}\right)} .
$$

Here $\kappa$ is an $\iota$-invariant Ricci-flat Kähler metric of $X, r(M)$ is the rank of $M$, $\tau(X / \iota, \kappa)$ is the Ray-Singer analytic torsion of orbifold $(X / \iota, \kappa)$ (or equivalently, the equivariant Ray-Singer analytic torsion of $(X, \iota, \kappa))$ and $\tau\left(X^{\iota},\left.\kappa\right|_{X^{\iota}}\right)$ is that of the fixed curve $\left(X^{\iota},\left.\kappa\right|_{X^{\iota}}\right)$. Note that $\operatorname{vol}\left(X^{\iota},\left.\kappa\right|_{X^{\iota}}\right)$ and $\tau\left(X^{\iota},\left.\kappa\right|_{X^{\iota}}\right)$ are defined multiplicatively with respect to the irreducible decomposition of divisors.

Theorem 1.1 (Theorems 3.4, 7.2, 7.3). Suppose that $r(M) \leq 17$.

(1) $\tau_{M}$ is independent of a choice of Ricci-flat Kähler metrics and defines an invariant of a 2-elementary $K 3$ surface of type $M$. It descents to a smooth $\Gamma_{M}$-invariant function on $\Omega_{M}^{0}:=\Omega_{M} \backslash \mathcal{D}_{M}$.

(2) There exists an automorphic form, $\Delta_{M}$, for $\Gamma_{M}$ of weight $(r(M)-6,4)$ such that $\tau_{M}=\left\|\Delta_{M}\right\|^{-1 / 4}$ and $\operatorname{div}\left(\Delta_{M}\right)=\mathcal{D}_{M}$.

(3) If $\delta$ is a root of $M^{\perp}$ and $\langle M \oplus \delta\rangle$ is the smallest 2-elementary lattice containing $M$ and $\delta$, then $\Delta_{\langle M \oplus \delta\rangle}$ coincides with the regularized restriction of $\Delta_{M}$ to $\Omega_{\langle M \oplus \delta\rangle}$.

It may be worth mentioning that analogous statment is valid for theta divisors of dimension $\geq 1$ ([Yo2]). By (3), any $\Delta_{M}$ is the regularized restriction of such $\Delta_{S}$ that $S$ is root free. Therefore, it is important to know an explicit formula for them. By Nikulin's classification, there are three such lattices up to isometry; $S \cong A_{1}, \mathrm{II}_{1,1}(2), \mathrm{II}_{1,9}(2)$.

Theorem 1.2 (Theorems 10.1, 10.2, 9.3).

(1) $\Delta_{A_{1}}^{15}=\Delta_{6}^{8} / \Psi_{A_{1}^{\perp}}$ where $\Delta_{6}$ is the discriminant of plane sextic curves, and $\Psi_{A_{1}^{\perp}}$ is Borcherds's product attached to the transcendental lattice and $\Theta_{E_{7}}(\tau) / \Delta(\tau)$. Here, $\Theta_{E_{7}}(\tau)$ is the vector valued theta series of $E_{7}$-lattice and $\Delta(\tau)$ is the Jacobi $\Delta$ function.

(2) $\Delta_{\mathrm{II}_{1,1}(2)}^{17}=\Delta_{4,4}^{9} / \Psi_{\mathrm{II}_{1,1}(2)^{\perp}}$ where $\Delta_{4,4}$ is the discriminant of the linear system of bidegree $(4,4)$ on $\mathbb{P}^{1} \times \mathbb{P}^{1}$ and $\Psi_{\mathrm{II}_{1,1}(2) \perp}$ is Borcherds's product attached to the transcendental lattice and $\eta(\tau)^{-8} \eta(2 \tau)^{-8}$. Here, $\eta(\tau)$ is the Dedekind $\eta$-function.

(3) $\Delta_{\mathrm{II}_{1,9}(2)}$ coincides with the Borcherds $\Phi$-function associated to the fake monster Lie superalgebra of rank 10 up to a constant, and $\Delta_{\mathrm{II}_{1,9}(2)}^{2}$ is Borcherds's product attached to $\mathrm{I}_{2,10}(2)$ and $\eta(\tau)^{-8} \eta(2 \tau)^{8} \eta(4 \tau)^{-8}$.

For the precise meaning of Borcherds's products used in this paper, see Theorem 8.1 and Definition 8.1. Concerning (3), another product formula can be found in $[\mathrm{Bo} 5]$. Jorgenson-Kramer $([\mathrm{J}-\mathrm{K}])$ treat Borcherds's $\Phi$-function from the view point of Green currents. Since Lefschetz fixed point formula in Alakelov theory is established by Köhler-Roessler recently $([\mathrm{K}-\mathrm{R}])$, it will be possible to represent $\Delta_{M}$ by Green currents.

Among all the 2-elementary lattices, there are some interesting ones from the view point of Nikulin's classification. As in the case of $\mathrm{II}_{1,9}(2), \Delta_{M}$ for them is closely related to Borcherds's theory ([Bo1-5]). 
Theorem 1.3 (Corollary 6.1, Theorems 8.2, 8.3, 9.4, 9.6).

(1) If $M \cong \mathrm{II}_{1,1} \oplus E_{8}(-2)$, one has $\Delta_{M}^{3}=C_{M} \Psi_{M^{\perp}} \otimes j_{M}^{*}\left(\Delta_{1} \Delta_{2}\right)$ where $\Delta_{i}$ is the Jacobi $\Delta$-function in the $i$-th variable on $\mathcal{A}_{1} \times \mathcal{A}_{1}, C_{M}$ is a constant, and $\Psi_{M \perp}$ is the denominator function of a generalized Kac-Moody superalgebra. Moreover, $\Psi_{M^{\perp}}$ is Borcherds's product attached to the transcendental lattice and $\Theta_{\Lambda_{16}}(\tau) / \Delta(\tau)$. Here, $\Theta_{\Lambda_{16}}(\tau)$ is the vector valued theta series of the Barnes-Wall lattice.

(2) If $g(M)=0$, namely $M^{\perp} \cong \mathrm{I}_{2,20-r(M)}(2) \quad(11 \leq r(M) \leq 17), \Delta_{M}$ is the denominator function of a generalized Kac-Moody superalgebra up to a constant. Moreover, $\Delta_{M}^{2}$ is Borcherds's product attached to the transcendental lattice and $\eta(\tau)^{-8} \eta(2 \tau)^{8} \eta(4 \tau)^{-8} \theta_{A_{1}}(\tau)^{r(M)-10}$ where $\theta_{A_{1}}(\tau)$ is the theta series of $A_{1}$-lattice.

In view of Theorems $1.2,1.3$, it seems natural to conjecture that any $\Delta_{M}$ in Theorem 1.1 is represented by Borcherds's product and discriminant of curves. Compared to fake monster Lie algebras, generalized Kac-Moody superalgebras appearing in Theorem 1.3 are not well understood. We shall show in Theorem 11.1 and Corollary 11.1 that product of certain 10 theta functions on the domain of type $I_{2,2}$ ([Ma]) admits Borcherds's product which is analogous to Gritsenko-Nikulin's product formula for Igusa's modular form ([G-N1]). We remark that GritsenkoNikulin's $\Delta_{11}$ ([G-N3]) coincides with $\Delta_{M}$ in (2) if $r(M)=17$. It is a mystery why the Ray-Singer analytic torsion is related to Borcherds's theory. (See $[\mathrm{H}-\mathrm{M}]$ for a physical explanation.) In view of works by Bershadski-Cecotti-Ooguri-Vafa ([B-C$\mathrm{O}-\mathrm{V}])$ and Harvey-Moore ([H-M $]$ ), it may be more natural to study the Ray-Singer analytic torsion of Calabi-Yau 3-folds associated to 2-elementary $K 3$ surfaces. (See $[\mathrm{Bc}],[\mathrm{V}]$ for these Calabi-Yau 3-folds.)

Let us briefly describe the contents of this paper. In $\S 2$, we recall the theory of 2-elementary $K 3$ surfaces due to Nikulin ([Ni4]). In $\S 3$, we recall the theory of Quillen metrics due to Bismut, Gillet, Soulé ([B-G-S], [Bi]) and apply it to derive the variational formula of $\tau_{M}$ on $\Omega_{M}^{0}$. In $\S 4$, to study the degeneration of Ricciflat Kähler metrics along the discriminant locus, we recall apriori estimates of the Monge-Amperè equations due to Yau ([Ya]). Following Kobayashi's work ([Ko]), we apply them to construct an approximate Ricci-flat Kähler metrics for degenerations of type $I$. Using it, we shall show in $\S 5$ that Kronheimer's ALE instanton can be used to compute the singularity of $\tau_{M}$. In $\S 6$, we study the heat kernel of the ALE instanton and determine the singularity of $\tau_{M}$ along $\mathcal{D}_{M}$. Theorem 1.1 shall be proved in $\S 7$. We recall Borcherds's theory ([Bo1-5]) in $\S 8,9$ and apply it to determine the structure of $\Delta_{M}$ for some $M$. We compute some examples in $\S 10,11$.

Acknowledgements. The author is grateful to Professor R.E. Borcherds who kindly showed him how to construct $\Psi_{M^{\perp}}$ in $\S 6.2$ from his $\Phi$-function of rank 26, and modular forms of type $\rho_{M}$ from those of higher level, to Professor S. Kondō from whom he learned the moduli space of lattice polarized $K 3$ surfaces, to Professor R. Kobayashi for teaching him degenerations of Ricci-flat Kähler metrics, and to Professor V.V. Nikulin and Doctor H. Imura for teaching him generalized KacMoody algebras. Without their helps, the present version would not exist. Together with above mentioned people, he is also grateful to Professors A. Fujiki, A. Matsuo, T. Ohsawa, K. Saito for discussions and suggestions. 


\section{$\S 2$. 2-Elementary K3 Surfaces and its Moduli Space}

2.1 Moduli Space of $K \mathbf{3}$ Surfaces. A compact complex surface $X$ is a $K 3$ surface if and only if $q(X)=0$ and $K_{X} \cong \mathcal{O}_{X}$. The cohomology lattice $H^{2}(X, \mathbb{Z})$ together with the intersection product is isometric to $L_{K 3}=\mathrm{II}_{1,1}^{3} \oplus E_{8}(-1)^{2}$ where $E_{8}$ is the positive definite $E_{8}$-lattice. (For a lattice $M, M(k)$ denotes the lattice with $\langle,\rangle_{M(k)}=k\langle,\rangle_{M}, \Delta(M)=\left\{m \in M ;\langle m, m\rangle_{M}=-2\right\}$ the root of $M$, and $M_{\mathbb{R}}$ (resp. $M_{\mathbb{C}}$ ) the vector space $M \otimes \mathbb{R}$ (resp. $M \otimes \mathbb{C}$ ). A sublattice $M^{\prime} \subset M$ is said to be primitive if $M / M^{\prime}$ is torsion free.) We denote by $\operatorname{Pic}_{X}:=H^{1,1}(X, \mathbb{R}) \cap H^{2}(X, \mathbb{Z})$ the Picard lattice and by $\Delta(X)$ its root. By the Riemann-Roch theorem, $\Delta(X)=$ $\Delta^{+}(X) \bigsqcup-\Delta^{+}(X)$ where $\Delta^{+}(X)$ consists of effective classes. The Kähler cone of $X$ is denoted by $C_{X}^{+}:=\left\{\kappa \in H^{1,1}(X, \mathbb{R}) ;\langle\kappa, \delta\rangle>0, \forall \delta \in \Delta^{+}(X)\right\}$ and its closure in $H^{1,1}(X, \mathbb{R})$ by $C_{X}$. Let $\phi_{X}: H^{2}(X, \mathbb{Z}) \rightarrow L_{K 3}$ be an isometry. A pair $\left(X, \phi_{X}\right)$ is said to be a marked $K 3$ surface. Two marked $K 3$ surfaces $\left(X, \phi_{X}\right)$ and $\left(X^{\prime}, \phi_{X^{\prime}}\right)$ are isomorphic when there exists an isomorphism $f: X \rightarrow X^{\prime}$ such that $\phi_{X} \circ f^{*}=\phi_{X^{\prime}}$. There exist the fine moduli space of marked $K 3$ surfaces. We briefly recall it ([P-S-S], [B-R], [B-P-V-V], [Be], [Mo], [Ni2,3] etc.). Define $\Omega$ by

$$
\Omega:=\left\{[\eta] \in \mathbb{P}\left(L_{K 3, \mathbb{C}}\right) ;\langle\eta, \eta\rangle_{L_{K 3}}=0,\langle\eta, \bar{\eta}\rangle_{L_{K 3}}>0\right\}
$$

For $\eta \in \Omega$, let $C(\eta)=\left\{x \in L_{K 3, \mathbb{R}} ;\langle x, \eta\rangle_{L_{K 3}}=0,\langle x, x\rangle_{L_{K 3}}>0\right\}$ be the positive cone over $\eta$ which consists of two connected components; $C(\eta)=C(\eta)^{+} \bigsqcup C(\eta)^{-}$. Let $S(\eta):=L_{K 3} \cap \eta^{\perp}$ be the Picard lattice over $\eta, \Delta(\eta)$ the root of $S(\eta)$, and $h_{\delta}:=$ $\left\{x \in L_{K 3, \mathbb{R}} ;\langle x, \delta\rangle_{L_{K 3}}=0\right\}$ the hyperplane orthogonal to $\delta$. Let $C(\eta) \backslash \bigcup_{\delta \in \Delta(\eta)} h_{\delta}=$ $\bigsqcup_{P} C_{P}$ be the decomposition into the connected components. $P$ is parametrized by the pair $\left(C(\eta)^{+}, \Delta_{P}^{+}(\eta)\right)$ where $\Delta_{P}^{+}(\eta)$ is a partition $\Delta(\eta)=\Delta_{P}^{+}(\eta) \bigsqcup-\Delta_{P}^{+}(\eta)$ with the following property:

(P1) If $\delta_{1}, \cdots, \delta_{k} \in \Delta_{P}^{+}(\eta)$ and $\delta=\sum_{i} n_{i} \delta_{i}\left(n_{i} \geq 0\right)$, then $\delta \in \Delta_{P}^{+}(\eta)$.

Set $K \Omega:=\left\{(\eta, \kappa) \in \Omega \times L_{K 3, \mathbb{R}} ; \kappa \in C(\eta)\right\}$ and

$$
K \Omega^{0}:=\left\{(\eta, \kappa) \in K \Omega ; \kappa \in C(\eta) \backslash \cup_{\delta \in \Delta(\eta)} h_{\delta}\right\}, \quad \tilde{\Omega}:=K \Omega^{0} / \sim .
$$

Here $(\eta, \kappa) \sim\left(\eta^{\prime}, \kappa^{\prime}\right)$ when $\eta=\eta^{\prime}$ and $\kappa, \kappa^{\prime} \in C_{P}(\eta)$ for some $P$. We denote by $p: K \Omega^{0} \rightarrow \tilde{\Omega}$ the projection map. Let $\pi: \tilde{\Omega} \rightarrow \Omega$ be the projection to the first factor. It is immediate by above construction that (1) $\tilde{\Omega}$ is a non-separated smooth analytic space, $(2) \pi$ is étale and surjective, $(3) \pi^{-1}(\eta)=\{P\}$; the set of all pairs $\left(C(\eta)^{+}, \Delta(\eta)^{+}\right)$where $\Delta(\eta)^{+}$satisfies $(\mathbf{P} \mathbf{1})$.

For $(\eta, \kappa) \in K \Omega^{0}$, put $C(\eta)_{\kappa}^{+}$for the connected component containing $\kappa$, and $\Delta(\eta)_{\kappa}^{+}:=\{\delta \in \Delta(\eta) ;\langle\kappa, \delta\rangle>0\}$. Then, $[(\eta, \kappa)] \in \tilde{\Omega}$ corresponds to $\left(\eta, C(\eta)_{\kappa}^{+}, \Delta(\eta)_{\kappa}^{+}\right)$.

Let $\eta_{X}$ be a symplectic form; $H^{0}\left(X, K_{X}\right)=\mathbb{C} \eta_{X}$. Then, $\pi\left(X, \phi_{X}\right):=\left[\phi_{X}\left(\eta_{X}\right)\right] \in$ $\mathbb{P}\left(L_{K 3, \mathbb{C}}\right)$ is the period of $\left(X, \phi_{X}\right)$ which lies on $\Omega$. Let $\kappa_{X}$ be a Kähler metric of $X$ and identify it with its class in $H^{2}(X, \mathbb{R})$. The pair $\left(X, \kappa_{X}\right)$ is said to be a polarized $K 3$ surface and the triplet $\left(X, \phi_{X}, \kappa_{X}\right)$ a marked polarized $K 3$ surface. The polarized period point of $\left(X, \phi_{X}, \kappa_{X}\right)$ is defined by $\pi\left(X, \phi_{X}, \kappa_{X}\right):=$ $\left(\left[\phi_{X}\left(\eta_{X}\right)\right], \phi_{X}\left(\kappa_{X}\right)\right) \in K \Omega^{0}$ and the Burns-Rapoport period point by $\left[\pi\left(X, \phi_{X}, \kappa_{X}\right)\right] \in$ $\tilde{\Omega}$.

Theorem 2.1. There exists the universal family of marked $K 3$ surfaces over $\tilde{\Omega}$; $\mathcal{P}: \mathcal{X} \rightarrow \tilde{\Omega}$, such that the period map coincides with $\pi: \tilde{\Omega} \rightarrow \Omega$.

Let $M$ be a primitive hyperbolic sublattice of $L_{K 3}$ with signature $(1, k)$. A marked $K 3$ surface $\left(X, \phi_{X}\right)$ is marked (ample) $M$-polarized if $\phi_{X}\left(\operatorname{Pic}_{X}\right) \supset M$ (and 
$\left.\phi_{X}\left(C_{X}^{+}\right) \cap M_{\mathbb{R}} \neq \emptyset\right)$. As $M$ is hyperbolic, a marked $M$-polarized $K 3$ surface is projective. Let $N:=M^{\perp}=\left\{l \in L_{K 3} ;\langle l, M\rangle=0\right\}$ be the orthogonal compliment of $M$ whose signature is $(2,19-k)$. By definition, the period point of a marked $M$-polarized $K 3$ surface is contained in the following subset of $\Omega$;

$$
\Omega_{M}:=\left\{[\eta] \in \mathbb{P}\left(N_{\mathbb{C}}\right) ;\langle\eta, \eta\rangle_{N}=0,\langle\eta, \bar{\eta}\rangle_{N}>0\right\} .
$$

Put $C(M):=\left\{x \in M_{\mathbb{R}} ;\langle x, x\rangle_{M}>0\right\}$ or equivalently $C(M)=C(\eta) \cap M_{\mathbb{R}}$. Define

$$
K \Omega_{M}:=\left\{([\eta], \kappa) \in K \Omega ;[\eta] \in \Omega_{M}, \kappa \in C(M)\right\}, \quad K \Omega_{M}^{0}:=K \Omega^{0} \cap K \Omega_{M} .
$$

Set $\tilde{\Omega}_{M}^{0}:=K \Omega_{M}^{0} / \sim$ and $\tilde{\Omega}_{M}$ for the closure of $\tilde{\Omega}_{M}^{0}$ in $\tilde{\Omega}$.

Lemma 2.1. For any $\eta \in \Omega_{M}, M \cap C(M) \backslash \bigcup_{\delta \in \Delta(\eta) \backslash \Delta(N)} h_{\delta} \neq \emptyset$.

Proof. If $M_{\mathbb{Q}} \cap C(M) \backslash \bigcup_{\delta \in \Delta(\eta) \backslash \Delta(N)} h_{\delta}=\emptyset$, then $C(M) \backslash \bigcup_{\delta \in \Delta(\eta) \backslash \Delta(N)} h_{\delta}=\emptyset$ and thus there exists $\delta \in \Delta(\eta) \backslash \Delta(N)$ such that $M_{\mathbb{R}} \subset h_{\delta}$. This implies that $\delta \in M^{\perp}=N$ and contradicts the choice of $\delta$.

Let $H_{l}:=\left\{[\eta] \in \Omega ;\langle\eta, l\rangle_{L_{K 3}}=0\right\}$ be the hyperplane defined by $l \in L_{K 3}$. We define the discriminant locus and an open subset by

$$
\mathcal{D}_{M}:=\cup_{\delta \in \Delta(N)} H_{\delta}, \quad \Omega_{M}^{0}:=\Omega_{M} \backslash \mathcal{D}_{M} .
$$

Let $\pi_{M}: \tilde{\Omega}_{M}^{0} \rightarrow \Omega_{M}^{0}$ be the period map restricted to $\tilde{\Omega}_{M}^{0}$. By Theorem 2.1 and Lemma 2.1, we get the following. (See $[\mathrm{Ni2}, 3]$ for the detail.)

Theorem 2.2. $\tilde{\Omega}_{M}^{0}$ is the fine moduli space of marked ample $M$-polarized $K 3$ surfaces whose period map is $\pi_{M}$. The universal family $\mathcal{P}_{M}: \mathcal{X}_{M}^{0} \rightarrow \tilde{\Omega}_{M}^{0}$ is obtained by putting $\mathcal{X}_{M}^{0}:=\left.\mathcal{X}\right|_{\tilde{\Omega}_{M}^{0}}$ and $\mathcal{P}_{M}:=\left.\mathcal{P}\right|_{\mathcal{X}_{M}^{0}}$.

We are now interested in the boundary of $\tilde{\Omega}_{M}^{0}$ in $\tilde{\Omega}_{M}$ and the family of marked $M$-polarized $K 3$ surfaces over it. For $\delta \in \Delta(N)$, put $H_{\delta}^{0}:=H_{\delta} \backslash \bigcup_{d \in \Delta(N) \backslash\{ \pm \delta\}} H_{d}$.

Lemma 2.2. Let $\delta \in \Delta(N), \eta \in H_{\delta}^{0}$ and $\kappa \in M \cap C(M) \backslash \bigcup_{d \in \Delta(\eta) \backslash \Delta(N)} h_{d}$. Then, $\Delta(\eta)=\Delta_{\kappa}^{+}(\eta) \bigsqcup-\Delta_{\kappa}^{+}(\eta) \bigsqcup\{ \pm \delta\}$ where $\Delta_{\kappa}^{+}(\eta)=\{d \in \Delta(\eta) ;\langle\kappa, d\rangle>0\}$. We put $\Delta_{1}^{+}(\eta):=\Delta_{\kappa}^{+}(\eta) \bigsqcup\{\delta\}$ and $\Delta_{2}^{+}(\eta):=\Delta_{\kappa}^{+}(\eta) \bigsqcup\{-\delta\}$. Then, $\Delta_{i}^{+}(\eta)$ satisfies $(\mathbf{P})$.

Proof. Suppose that $d \in \Delta(\eta) \backslash \Delta(N)$. Then, either $\langle\kappa, d\rangle>0$ or $\langle\kappa, d\rangle<0$ because $\kappa \notin \bigcup_{\delta^{\prime} \in \Delta(\eta) \backslash \Delta(N)} h_{\delta^{\prime}}$. This implies $\Delta(\eta) \backslash \Delta(N)=\Delta_{\kappa}^{+}(\eta) \bigsqcup-\Delta_{\kappa}^{+}(\eta)$. Suppose that $d \in \Delta(\eta) \cap \Delta(N)$ which means $d \in \Delta(N)$ and $\eta \in H_{d}$. By the choice of $\eta$, $d= \pm \delta$ which prove the first assertion. Since the Weyl group $W(\eta)$ acts properly discontinuously on $C(\eta)$, there exists a small neighborhood $K$ of $\kappa$ in $C(\eta)$ such that $s_{d}(K) \cap K \neq \emptyset$ for $d \in \Delta(\eta)$ means $d= \pm \delta$ where $s_{d}$ is the reflection in $d$. Thus there exists $\epsilon_{0}>0$ such that for any $0<\epsilon<\epsilon_{0}, \kappa+\epsilon \delta \in V(\eta) \backslash \bigcup_{d \in \Delta(\eta)} h_{d}$. Clearly, $\Delta_{\kappa}^{+}(\eta) \bigsqcup\{-\delta\}=\{d \in \Delta(\eta) ;\langle\kappa+\epsilon \delta, d\rangle>0\}$. Similarly, $\Delta_{1}^{+}(\eta)$ is the partition associated to $\kappa-\epsilon \delta$.

Let $D$ be the unit disc and $\gamma: D \rightarrow \Omega_{M}$ be a holomorphic curve which intersects transversally $H_{\delta}^{0}$ at $\eta_{0}=\gamma(0)$. For $\eta_{0}$, let $\kappa$ and $P_{i}=\left(C\left(\eta_{0}\right)_{\kappa}^{+}, \Delta_{i}^{+}\left(\eta_{0}\right)\right)$ be the same as in Lemma 2.2. Set $\kappa_{1}:=\kappa-\epsilon \delta$ and $\kappa_{2}:=\kappa+\epsilon \delta$. Let $\tau_{i}(i=1,2)$ be the points in $\tilde{\Omega}_{M}$ such that $\tau_{i}=\left(\eta_{0}, C\left(\eta_{0}\right)^{+}, \Delta_{i}^{+}\left(\eta_{0}\right)\right)$. Let $U$ be a small neighborhood of $\eta_{0}$ in $\Omega_{M}$, and $U_{i}$ a neighborhood of $\tau_{i}$ in $\tilde{\Omega}_{M}$ such that $\pi: U_{i} \rightarrow U$ is an isomorphism. Let $\gamma_{i}:=\left(\left.\pi\right|_{U_{i}}\right)^{-1} \circ \gamma: D \rightarrow U_{i}$ be the lift of $\gamma$ to $U_{i}$ such that $\gamma_{i}(0)=\tau_{i}$. Let $\left(X_{t}^{i}, \phi_{t}^{i}\right)$ be the marked $K 3$ surface corresponding to $\gamma_{i}(t)$ and $\eta_{t}=\pi\left(X_{t}^{i}, \phi_{t}^{i}\right)$ its period. From construction and Burns-Rapoport's lemma ([B-R], [Mo, pp.306]), it follows that $\left(\phi_{t}^{i}\right)^{-1}(\kappa)$ is an ample class of $X_{t}^{i}$ and $\gamma_{1}(t)=\gamma_{2}(t)$ for $t \neq 0$. 
Lemma 2.3. Let $C_{\delta}^{1}$ (resp. $C_{-\delta}^{2}$ ) be the effective cycle on $X_{0}^{1}$ (resp. $X_{0}^{2}$ ) such that $\phi_{0}^{1}\left(C_{\delta}^{1}\right)=\delta$ (resp. $\phi_{0}^{2}\left(C_{-\delta}^{2}\right)=-\delta$ ). Then, $C_{\delta}^{1}$ (resp. $\left.C_{-\delta}^{2}\right)$ is an irreducible -2 curve.

Proof. We only prove the case $i=1$. Since $\langle\kappa, d\rangle \geq 0$ for any $d \in \Delta_{1}^{+}\left(\eta_{0}\right)$ and $\langle\kappa, \kappa\rangle>0,\left(\phi_{0}^{1}\right)^{-1}(\kappa)$ is a pseudo-ample class $([\mathrm{Mo}, \S 5])$. As $\langle\kappa, \delta\rangle=0, C_{\delta}^{1}$ consists of a chain of -2-curves by Mayer's theorem ([Mo, $\S 5])$. Since $C_{\delta}^{1}$ is effective, we can write $C_{\delta}^{1}=\sum_{j} m_{j} E_{j}$ where $E_{j}$ is an irreducible -2-curve such that $\left\langle\kappa, \phi_{0}^{1}\left(E_{j}\right)\right\rangle=0$, and $m_{i} \in \mathbb{Z}_{+}$. Suppose $C_{\delta}^{1}$ is not irreducible. Then, $E_{1} \subsetneq C_{\delta}^{1}$. This implies $\phi_{0}^{1}\left(E_{1}\right) \in \Delta\left(\eta_{0}\right) \cap \kappa^{\perp}$. By Lemma 2.2 and effectivity of $E_{1}, \phi_{0}^{1}\left(E_{1}\right)=\delta$. Thus, $C_{\delta}^{1}=E_{1}$ and contradicts the assumption.

Let $\pi_{i}: \gamma_{i}^{*} \mathcal{X} \rightarrow D$ be the pullback of the universal family by $\gamma_{i}$. Recall that a compact complex surface with at most rational double points is said to be a generalized $K 3$ surface when its minimal resolution is a $K 3$ surface.

Proposition 2.1. There exists a family of generalized K3 surface $\pi: \mathcal{Y} \rightarrow D$, a contraction morphism $b_{i}: \gamma_{i}^{*} \mathcal{X} \rightarrow \mathcal{Y}$ which commutes with the projections, and a birational map e $: \gamma_{1}^{*} \mathcal{X} \rightarrow-\rightarrow \gamma_{2}^{*} \mathcal{X}$ such that

(1) $b_{1}$ (resp. $\left.b_{2}\right)$ is the blow-down of $C_{\delta}^{1}$ (resp. $\left.C_{-\delta}^{2}\right)$ to a point o,

(2) $e: X_{0}^{1} \backslash C_{\delta}^{1} \rightarrow X_{0}^{2} \backslash C_{-\delta}^{2}$ is an isomorphism such that $b_{1}=b_{2} \circ e$,

(3) $e$ is the identity map on $X_{t}$ for any $t \neq 0$,

(4) On $X_{1,0}$ and $X_{2,0}, \phi_{1} \circ e^{*}=w_{\delta} \circ \phi_{2}$ where $w_{\delta}$ is the reflection in $\delta$.

(5) $(\mathcal{Y}, o)$ and $\left(Y_{0}, o\right)$ are nodes of dimension 3 and 2 respectively.

Proof. See [Mo, §3 Cor.2] and [Be, pp.143 Remarques].

Lemma 2.4. There exists an embedding $j: \mathcal{Y} \rightarrow \mathbb{P}^{N} \times D$ such that (1) $\pi=p r_{2} \circ j$ (2) $\phi_{t}^{i}\left(c_{1}\left(b_{i}^{*} \mathcal{O}_{\mathbb{P}^{N}}(1)\right)\right)=m \kappa$ for some $m \in \mathbb{Z}_{+}$and any fiber of $\pi_{i}$.

Proof. Let $L_{i} \rightarrow \gamma_{i}^{*} \mathcal{X}$ be the holomorphic line bundle such that $\phi_{t}^{i}\left(c_{1}\left(L_{i}\right)\right)=\kappa$ for any fiber of $\pi_{i}$. There exists $m \gg 1$ such that the linear system $\left|L_{i}\right|$ is very ample on $X_{t}^{i}$ for $t \neq 0$, and is base point free and an embedding modulo $C_{\delta}$; $\Phi_{\left|m L_{i}\right|} \times \pi_{i}: \gamma^{*} \mathcal{X} \hookrightarrow \mathbb{P}^{N} \times D$. By construction and Proposition 2.1, we get $\mathcal{Y}=\left(\Phi_{\left|m L_{i}\right|} \times \pi_{i}\right)\left(\gamma_{i}^{*} \mathcal{X}\right)$ and $L_{i}^{m}=\Phi_{\left|m L_{i}\right|}^{*} \mathcal{O}_{\mathbb{P}^{N}}(1)$.

2.2 2-Elementary $K 3$ Surfaces and 2-Elementary Lattices. For a lattice $M$, we denote by $M^{\vee}$ its dual lattice relative to the quadratic form of $M . A(M):=$ $M^{\vee} / M$ is called the discriminant group. A lattice $M$ is said to be 2-elementary when $A(M) \cong(\mathbb{Z} / 2 \mathbb{Z})^{l(M)}$ for some $l(M) \in \mathbb{Z}_{\geq 0}$. We denote by $r(M)$ the rank of $M$. Let $\delta(M)$ be the parity of the discriminant form ([Ni1,4]). By Nikulin, the triplet $(r, l, \delta)$ determines the isometry class of 2-elementary lattices $([\mathrm{Ni} 1])$.

Let $M$ be a primitive hyperbolic 2-elementary lattice of $L_{K 3} . \quad N$ is also 2elementary and there exists a natural isomorphism between $A(M)$ and $A(N)$. Consider the sublattice $L^{\prime}:=M \oplus N$ and the involution $I_{M}$ on $L^{\prime}$;

$$
I_{M}(x, y)=(x,-y) \quad(x \in M, y \in N)
$$

As $I_{M}$ uniquely extends to an involution of $L_{K 3}$, identify $I_{M}$ with the extended one. By construction, $M$ is the fixed part and $N$ is the anti-fixed part;

$$
M=\left\{l \in L ; I_{M}(l)=l\right\}, \quad N=\left\{l \in L ; I_{M}(l)=-l\right\} .
$$


$I_{M}$ induces an involution on $\tilde{\Omega}$ by $I\left(X, \phi_{X}, \kappa_{X}\right)=\left(X, I_{M} \circ \phi_{X}, \kappa_{X}\right)$ where $\left(X, \phi_{X}, \kappa_{X}\right)$ is a marked polarized $K 3$ surface. Let $\Phi: R^{2} \mathcal{P}_{*} \mathbb{Z} \rightarrow L_{K 3}$ be the global trivialization of the second cohomology group. (Thus $\left.\Phi\right|_{\left(X, \phi_{X}\right)}=\phi_{X}$.) By the universality, there exists an involution $\iota_{\mathcal{X}}: \mathcal{X} \rightarrow \mathcal{X}$ such that the following diagrams commute;
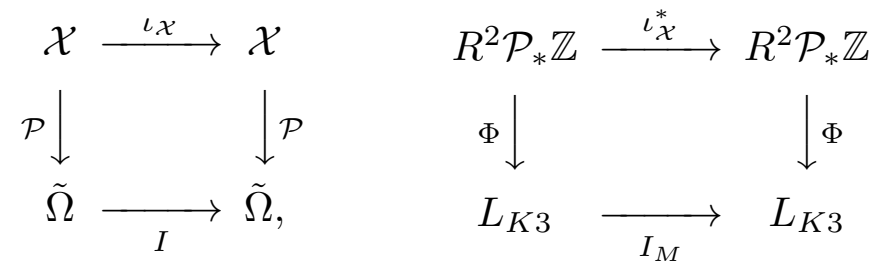

By construction, $\tilde{\Omega}_{M}^{0}$ is contained in the fixed locus of $I$. Put $\iota_{M}:=\iota_{\mathcal{X}} \mid \mathcal{X}_{M}$. By (2.8), $\iota_{M}$ is an involution over $\mathcal{X}_{M}^{0}$ which induces an involution on each fiber $\left(X, \phi_{X}\right)$ such that $\phi_{X} \circ \iota_{M}^{*}=I_{M} \circ \phi_{X}$. In this way, associated to the 2-elementary primitive hyperbolic lattice $M$, any fiber of $\mathcal{X}_{M}^{0}$ is a $K 3$ surface with an antisymplectic involution. Here, an involution $\iota: X \rightarrow X$ is anti-symplectic when $\iota^{*} \eta_{X}=-\eta_{X}$. For a pair $(X, \iota)$ of $K 3$ surface and its anti-symplectic involution, put $H^{2}(X, \mathbb{Z})_{ \pm}=\left\{l \in H^{2}(X, \mathbb{Z}) ; \iota_{X}^{*}(l)= \pm l\right\}$.

Definition 2.1. A pair $\left(X, \iota_{X}\right)$ of a $K 3$ surface and an anti-symplectic involution is said to be a 2-elementary $K 3$ surface of type $M$ if there exists a marking $\phi_{X}$ such that $\phi_{X} \circ \iota_{X}^{*} \circ \phi_{X}^{-1}=I_{M}$, or equivalently $\phi_{X}\left(H^{2}(X, \mathbb{Z})_{+}\right)=M$. Two 2-elementary K3 surfaces $(X, \iota),\left(X^{\prime}, \iota^{\prime}\right)$ are isomorphic if there exists an isomorphism $f: X \rightarrow$ $X^{\prime}$ such that $f \circ \iota=\iota^{\prime} \circ f$. Marking as above is said to be a marking of 2-elementary K3 surfaces of type $M$.

The following is clear by Theorem 2.2 and above argument. (See [Ni2-4].)

Theorem 2.3. $\tilde{\Omega}_{M}^{0}$ is the fine moduli space of marked 2-elementary $K 3$ surface of type $M$ and $\mathcal{P}_{M}:\left(\mathcal{X}_{M}^{0}, \iota_{M}\right) \rightarrow \tilde{\Omega}_{M}^{0}$ is the universal family.

Let $\Gamma(M)$ (resp. $\left.\Gamma_{M}\right)$ be the following subgroup of $O\left(L_{K 3}\right)(\operatorname{resp} . \mathrm{O}(\mathrm{N}))$;

$$
\Gamma(M):=\left\{g \in O(L), I_{M} \circ g=g \circ I_{M}\right\}, \quad \Gamma_{M}:=\left\{\left.g\right|_{N} ; g \in \Gamma(M)\right\}
$$

Then, $\Gamma(M)$ acts on $\tilde{\Omega}_{M}^{0}$ and $\Gamma_{M}$ acts on $\Omega_{M}^{0}$ by $g \cdot[(\eta, \kappa)]:=[(g \eta, g \kappa)]$ and $\left(\left.g\right|_{N}\right) \eta=g \eta$ respectively. By the global Torelli theorem, two 2-elementary $K 3$ surface of type $M$ are isomorphic if their period points in $\tilde{\Omega}_{M}^{0}$ lie on the same $\Gamma(M)$-orbit. The following theorem can be proved in the same manner as the proof of weak Torelli theorem and surjectivity theorem for the period map of Enriques surfaces ([Na, Corollary 4.14 and Theorem 7.1]).

Theorem 2.4. Via the period $\operatorname{map} \pi_{M}, \tilde{\Omega}_{M}^{0} / \Gamma(M)=\Omega_{M}^{0} / \Gamma_{M}$.

Since $\Gamma_{M}$ is an arithmetic subgroup of $O(2,20-r(M))$, and $\Omega_{M}$ is two copies of the symmetric bounded domain of type IV, both $\Omega_{M} / \Gamma_{M}$ and $\Omega_{M}^{0} / \Gamma_{M}$ are quasiprojective algebraic variety by Baily-Borel. Define modular varieties by

$$
\mathcal{M}_{M}:=\Omega_{M} / \Gamma_{M}, \quad \mathcal{M}_{M}^{0}:=\Omega_{M}^{0} / \Gamma_{M}=\tilde{\Omega}_{M}^{0} / \Gamma(M) .
$$

By Theorem 2.4, $\mathcal{M}_{M}^{0}$ is the moduli space of 2-elementary $K 3$ surfaces of type $M$. To see what happens on the involution along the generic point of discriminant 
locus, let $\pi_{i}: \gamma_{i}^{*} \mathcal{X} \rightarrow D$ be the same as in Proposition 2.1, and $I, \iota \mathcal{X}$ as in (2.8). As $I \circ \gamma_{1}=\gamma_{2}$, it follows from the universal property of $\mathcal{P}: \mathcal{X} \rightarrow \tilde{\Omega}$ that $\iota_{\mathcal{X}}$ induces an isomorphism between $\left(\pi_{1}, \gamma_{1}^{*} \mathcal{X}, D\right)$ and $\left(\pi_{2}, \gamma_{2}^{*} \mathcal{X}, D\right)$. Namely, $I \circ \pi_{1}=\pi_{2} \circ \iota \mathcal{X}$. Thus, by Proposition 2.1, there exist two (rational) maps $e$ and $\iota \mathcal{X}$ between $\gamma_{1}^{*} \mathcal{X}$ and $\gamma_{2}^{*} \mathcal{X}$. Then, $\iota_{1}:=e^{-1} \circ \iota_{\mathcal{X}}: \gamma_{1}^{*} \mathcal{X} \backslash C_{\delta}^{1} \rightarrow \gamma_{1}^{*} \mathcal{X} \backslash C_{\delta}^{1}$ extends to a rational involution of $\gamma_{1}^{*} \mathcal{X}$ which commutes with the projection.

Proposition 2.2. There exists a holomorphic involution $\iota \mathcal{Y}: \mathcal{Y} \rightarrow \mathcal{Y}$ which commutes with the projection $\pi: \mathcal{Y} \rightarrow U$ such that $\iota_{\mathcal{X}}=b_{1}^{-1} \circ \iota_{\mathcal{Y}} \circ b_{1}$ on $\gamma_{1}^{*} \mathcal{X} \backslash C_{\delta}^{1}$. Namely, $\pi_{1}:\left.\gamma_{1}^{*} \mathcal{X}\right|_{D \backslash\{0\}} \rightarrow D \backslash\{0\}$ extends to the family $\pi: \mathcal{Y} \rightarrow D$ by contracting $C_{\delta}^{1}$. The central fiber $Y_{0}$ is a generalized $K 3$ surface with one node o on which $\iota y$ induces an anti-symplectic involution. Moreover, o is a fixed point of $\iota$.

Proof. Put $\iota \mathcal{Y}:=b_{1} \circ \iota_{1} \circ b_{1}^{-1}$. As $\iota_{1}$ is a regular involution over $\gamma_{1}^{*} \mathcal{X} \backslash C_{\delta}^{1}, \iota \mathcal{Y}$ is an involution over $\mathcal{Y} \backslash\{o\}$ where $o=b_{1}\left(C_{\delta}^{1}\right)$. By putting $\iota \mathcal{Y}(o)=o, \iota \mathcal{Y}$ extends to an involution over $\mathcal{Y}$. Since $(\mathcal{Y}, o)$ is normal by Proposition $2.1, \iota \mathcal{Y}$ is regular.

For a 2-elementary $K 3$ surface $(X, \iota)$ of type $M$, let $X^{\iota}$ be the fixed locus; $X^{\iota}:=\{x \in X ; \iota(x)=x\}$ consisting of disjoint union of finitely many smooth curves. Nikulin determined the topological type of $X^{\iota}([\mathrm{Ni} 4])$.

Theorem 2.5. Let $M$ be the lattice of Nikulin type $(r, l, \delta)$. Then,

$$
X^{\iota}=\left\{\begin{array}{lll}
(1) & \emptyset & (r, l, \delta)=(10,10,0) \\
(2) & C_{1}^{(1)}+C_{2}^{(1)} & (r, l, \delta)=(10,8,0) \\
(3) & C^{(g(M))}+\sum_{i=1}^{k(M)} E_{i} & (r, l, \delta) \neq(10,10,0),(10,8,0)
\end{array}\right.
$$

where $C^{(g)}$ is a smooth curve of genus $g$, and $E_{i}$ is a smooth -2-curve. In (3), $g(M)=(22-r-l) / 2$ and $k(M)=(r-l) / 2$.

Let $\pi: \mathcal{Y} \rightarrow U$ and $\iota \mathcal{Y}$ be the degenerating family of 2-elementary $K 3$ surfaces of type $M$ considered in Proposition 2.2. Set $Y_{0}$ for the central fiber. Since the embedding dimension of $\left(Y_{0}, o\right)$ is 3 and $o$ is a fixed point of $\iota \mathcal{y},\left(\iota \mathcal{Y}_{*}\right)_{o}$ induces an involution on $\mathbb{C}^{3}$. As $\iota \mathcal{Y}$ is anti-symplectic, $\operatorname{det}\left(\iota^{*}\right)_{O}=-1$ and $\left(\iota \mathcal{Y}_{*}\right)_{O}$ is expressed by the diagonal matrix with eigenvalues as follows in a suitable coordinates;

$$
\text { Type }(0,3): \quad(-1,-1,-1), \quad \text { Type }(2,1): \quad(1,1,-1) \text {. }
$$

As $(\mathcal{Y}, o)$ is a node, by a careful look at the morsification procedure, we get the following.

Proposition 2.3. Let $\mathcal{Z}$ be the fixed locus of $(\mathcal{Y}, \iota \mathcal{Y})$.

(1) In case of type $(0,3), o$ is an isolated point of $\mathcal{Z}$ and there exists a neighborhood $V$ of $o$ such that $\pi(x, y, z, t)=t$ and $\iota \mathcal{Y}(x, y, z, t)=(-x,-y,-z, t)$, $\left(\left.\mathcal{Y}\right|_{V}, o\right)=\left\{(x, y, z, t) ; x y-z^{2}-t^{2}=0\right\}$.

(2) In case of type $(2,1), \mathcal{Z}$ has a unique node at o and there exists a neighborhood $V$ of $o$ such that $\pi(x, y, z, t)=t$ and $\iota \mathcal{Y}(x, y, z, t)=(-x,-y,-z, t)$, $\left(\left.\mathcal{Y}\right|_{V}, o\right)=\left\{(x, y, z, t) ; x y-z^{2}-t^{2}=0\right\}$

For a 2-elementary primitive hyperbolic lattice $M$ and $\delta \in \Delta(N)$, set $\langle M \oplus \delta\rangle$ for the smallest 2-elementary primitive hyperbolic lattice containing $M$ and $\delta$. It follows from definition that $\Delta\left(\langle M \oplus \delta\rangle^{\perp}\right)=\delta^{\perp} \cap \Delta(N)$ and $H_{\delta} \cap \Omega_{M}=\Omega_{\langle M \oplus \delta\rangle}$. 
Lemma 2.5. For the family $\pi: \mathcal{Y} \rightarrow D$ in Proposition $2.2, \iota_{0}^{1}:=b_{1}^{-1}\left(\left.\iota \mathcal{Y}\right|_{Y_{0}}\right) b_{1}$ extends to a involution on the minimal resolution $X_{0}^{1}$, and $\left(X_{0}, \iota_{0}^{1}\right)$ becomes a 2 elementary $K 3$ surface of type $\langle M \oplus \delta\rangle$.

Proof. By Proposition 2.3, it is clear that $\iota_{0}^{1}$ extends to an involution on $X_{0}^{1}$. Let $\phi_{1}$ and $\phi_{2}$ be the marking as in Proposition 2.1. By Proposition 2.1 (4) and Definition 2.1 , we get $\phi_{1}^{-1}\left(\iota_{0}^{1}\right)^{*} \phi_{1}=\left(\phi_{1}^{-1} \iota_{M}^{*} \phi_{2}\right) \circ\left(\phi_{2}^{-1}\left(e^{-1}\right)^{*} \phi_{1}\right)=I_{M} \circ w_{\delta}$. Since $\delta \perp M$, we get $I_{M} \circ w_{\delta}=w_{\delta} \circ I_{M}$ and $I_{M} \circ w_{\delta}=I_{\langle M \oplus \delta\rangle}$.

Let $\mathcal{A}_{g}:=\mathfrak{S}_{g} / \operatorname{Sp}(2 g ; \mathbb{Z})$ be the Siegel modular variety. (When $g=0, \mathcal{A}_{g}$ is a point.) We denote by $\mathcal{A}_{g}^{*}$ and $\mathcal{M}_{M}^{*}$ the Satake-Baily-Borel compactification. Let $C=\sum_{i=1}^{l} C_{i}$ be a disjoint union of smooth curves and $\operatorname{Jac}\left(C_{i}\right)$ the Jacobi variety of $C_{i}$. Put $[\operatorname{Jac}(C)]:=\left(\left[\operatorname{Jac}\left(C_{1}\right)\right], \cdots,\left[\operatorname{Jac}\left(C_{l}\right)\right]\right) \in \mathcal{A}_{g_{1}} \times \cdots \times \mathcal{A}_{g_{l}}$ where $\left[\operatorname{Jac}\left(C_{i}\right)\right]$ is the period of $\operatorname{Jac}\left(C_{i}\right)$. By Theorems 2.4 and 2.5, we can define a morphism $j_{M}^{0}: \mathcal{M}_{M}^{0} \rightarrow \mathcal{A}_{g(M)}$ as follows.

Definition 2.2. $j_{M}^{0}: \mathcal{M}_{M}^{0} \ni\left[\left(X, \iota_{X}\right)\right] \longrightarrow\left[\operatorname{Jac}\left(X^{\iota}\right)\right] \in \mathcal{A}_{g(M)}$. When $(r, l, \delta)=$ $(10,10,0), j_{M}^{0}$ is defined to be the constant map. When $(r, l, \delta)=(10,8,0), j_{M}^{0}$ takes its value in $S^{2}\left(\mathcal{A}_{1}\right)$, the second symmetric product of $\mathcal{A}_{1}$.

Proposition 2.4. $j_{M}^{0}$ extends to a rational map $j_{M}: \mathcal{M}_{M}^{*-\rightarrow} \mathcal{A}_{g(M)}^{*}$ if $r \leq 17$.

Proof. Since $\mathcal{M}_{M}^{*}$ and $\mathcal{A}_{g(M)}^{*}$ are projective algebraic varieties, it is enough to show that $j_{M}$ extends to a morphism from an open subset $V$ of $\mathcal{M}_{M}$ such that codim $\mathcal{M}_{M} \backslash V \geq 2$ because the boundary components of $\mathcal{M}_{M}^{*}$ have codimension $\geq 2$ when $r(M) \leq 17$. Put $V=\mathcal{M}_{M}^{0} \cup \bigcup_{\delta \in \Delta(N)} H_{\delta}^{0} / \Gamma_{M}$. Take a point $\eta \in H_{\delta}^{0}$ and its small neighborhood $U$. By Proposition 2.2, we have a family of 2-elementary $K 3$ surfaces of type $M$ whose fiber over $U \cap H_{\delta}$ is a generalized $K 3$ surface with an anti-symplectic involution. Let $\left(X_{\eta}, \iota_{\eta}\right)$ be the corresponding generalized $K 3$ surface with involution over $\eta$. Let $X_{\eta}^{\iota}$ be the fixed locus and $\hat{X}_{\eta}^{\iota}$ its normalization. Define $j_{M}\left(X_{\eta}, \iota_{\eta}\right):=\left[\operatorname{Jac}\left(\hat{X}_{\eta}^{\iota}\right)\right] \in \mathcal{A}_{g(M)}^{*}$. Since $X_{\eta}^{\iota}$ has at most a node, it is well defined and gives an extension of $j_{M}$ to $U$. As $j_{M}$ is defined on $U \backslash H_{\delta}$, its extension to $U$ is unique. This proves that $j_{M}^{0}$ extends to $V$.

Proposition 2.5. For $\delta \in \Delta(N),\left.j_{M}\right|_{H_{\delta}^{0}}=j_{\langle M, \delta\rangle}$.

Proof. Take a point $\eta_{0} \in H_{\delta}^{0}$ and its small neighborhood $U$ in $\Omega_{M}$. Let $\tilde{U} \subset \tilde{\Omega}_{M}$ be a lift of $U$ such that $\tilde{\Omega}_{M}^{0}$ is dense in $\tilde{U}$. Let $\pi^{\prime}: \mathcal{Y} \rightarrow \tilde{U}$ and $\pi^{\prime}: \mathcal{Z} \rightarrow \tilde{U}$ be the family of 2-elementary $K 3$ surfaces and its fixed locus. By the period map, identify $U$ with $\tilde{U}$. Then, over $U \cap H_{\delta}^{0}$, a fiber of $\mathcal{Y}$ is a generalized $K 3$ surface with an anti-symplectic involution. Take $\tau \in U \cap H_{\delta}^{0}$. Let $\left(Y_{\tau}, \iota_{\tau}\right)$ be the fiber over $\tau$ and $Z_{\tau}$ its fixed locus. Let $p: \tilde{Y}_{\tau} \rightarrow Y_{\tau}$ be the minimal resolution of $Y_{\tau}$. By Lemma 2.5, $\tilde{\iota}_{\tau}:=p^{-1} \circ \iota \circ p$ extends to an involution over $\tilde{Y}_{\tau}$. Set $\tilde{Z}_{\tau}$ for the fixed locus of $\left(\tilde{Y}_{\tau}, \tilde{\iota}_{\tau}\right)$. By Proposition 2.3, $\tilde{Z}_{\tau}=Z_{\tau}+E$ when $(\mathcal{Y}, o)$ is of type $(0,3)$, and $\tilde{Z}_{\tau}=\hat{Z}_{\tau}$ is the normalization when $(\mathcal{Y}, o)$ is of type $(2,1)$. By definition, we find that $j_{\langle M, \delta\rangle}=\left.j\right|_{H_{\delta}^{0}}$ on $U$. 


\section{§3. An Invariant of 2-Elementary $K 3$ Surfaces}

3.1 Determinant Bundles and Quillen Metrics. Let $(M, g)$ be a compact Kähler manifold on which acts a finite group $G$ holomorphically and isometrically. Let $\square_{0, q}^{G}$ be the Laplacian restricted to the space of $G$-invariant $(0, q)$-forms and $\zeta_{0, q}^{G}(s)$ be the spectral zeta function of $\square_{0, q}^{G}$, which is regular at $s=0 . \zeta_{0, q}^{G}(s)$ is nothing but the spectral zeta function of the orbifold $(M / G, g)$.

Definition 3.1. The Ray-Singer analytic torsion of $(M / G, g)$ is defined by

$$
\tau(M / G, g):=\prod_{q \geq 0}\left(\operatorname{det} \square_{0, q}^{G}\right)^{(-1)^{q} q}, \quad \operatorname{det} \square_{0, q}^{G}:=\exp \left(-\left.\frac{d}{d s}\right|_{s=0} \zeta_{0, q}^{G}(s)\right) .
$$

Let $\pi: X \rightarrow S$ be a proper smooth morphism of Kähler manifolds on which acts the finite group $G$ holomorphically. Assume that $G$ preserves the fiber of $\pi$. An equivariant determinant of cohomology is a line bundle on $S$ defined by $\lambda(X / S, G):=\bigotimes_{q \geq 0}\left(\operatorname{det} R_{G}^{q} \pi_{*} \mathcal{O}_{X}\right)^{(-1)^{q}}$. Here the fiber at $s$ of the sheaf $R_{G}^{q} \pi_{*} \mathcal{O}_{S}$ is the equivariant cohomology group $H^{q}\left(X_{s}, \mathcal{O}_{X_{s}}\right)^{G}=H^{q}\left(X_{s} / G, \mathcal{O}_{X_{s} / G}\right)$. In the sequel, we assume that a $G$-invariant Kähler metrics $g_{X / S}$ is equipped on the relative tangent bundle $T X / S:=\operatorname{ker} \pi_{*}$. Namely, $g_{X / S}$ is a family of $G$-invariant Kähler metric $g_{t}:=\left.g_{X / S}\right|_{X_{t}}$. Via the Hodge theory, the fiber of $\pi$ is identified with the determinant of $G$-invariant harmonic forms which induces the $L^{2}$-metric on $\lambda(X / S, G)$. We denoted it by $\|\cdot\|_{L^{2}}^{G}$.

Definition 3.2. The Quillen metric of $\lambda(X / S, G)$ relative to $g_{X / S}$ is defined by $\|\cdot\|_{Q}^{2}(t):=\tau\left(X_{t} / G, g_{t}\right) \cdot\|\cdot\|_{L^{2}}^{G}(t)$.

Let $X_{g}=\{x \in X ; g(x)=x\}$ be the fixed locus of $g, R_{T X_{g} / S}$ the curvature form of $\left(T X_{g} / S,\left.g_{X / S}\right|_{T X_{g} / S}\right), R_{N_{X_{g} / X}}$ the curvature form of the relative normal bundle $\left(N_{X_{g} / X},\left.g_{X / S}\right|_{N_{X_{g} / X}}\right)$, and $\exp \left(i \theta_{j}\right)$ an eigenvalue of $g_{N_{X_{g} / X}}$. Let $\operatorname{Td}(A)$ and $e(A)$ be the Todd and Euler genuses i.e., $\operatorname{Td}(A)=\operatorname{det}\left(A\left(1-e^{-A}\right)^{-1}\right)$ and $e(A)=\operatorname{det}(A)$. The following theorems are due to Bismut ([Bi]) and Bismut-Gillet-Soulé ([B-G-S]). (Although detailed proof of Theorem 3.1 is not written in [B-G-S], it can be proved in the similar way as in the case $G=\{1\}$. Details are left to the reader. We refer to Köhler-Roessler's paper ([K-R]) for further generalizations.)

Theorem 3.1. The Chern form of $\lambda(X / S, G)_{Q}:=\left(\lambda(X / S, G),\|\cdot\|_{Q}\right)$ is given by

$$
c_{1}\left(\lambda(X / S, G)_{Q}\right)=\frac{1}{|G|} \sum_{g \in G} \pi_{*}\left(\operatorname{Td}_{g}\left(T X / S, g_{X / S}\right)\right)^{(1,1)} .
$$

Here $\operatorname{Td}_{g}\left(T X / S, g_{X / S}\right)$ is the $g$-Todd genus of $\left(T X / S, g_{X / S}\right)$;

$\operatorname{Td}_{g}\left(T X / S, g_{X / S}\right)=\operatorname{Td}\left(\frac{i}{2 \pi} R_{T X_{g} / S}\right) \prod_{j=1}^{q}(\mathrm{Td} / \mathrm{e})\left(\frac{i}{2 \pi} R_{N_{X_{g} / X}}+i \theta_{j}\right)$.

Theorem 3.2. Let $g_{X / S}$ and $g_{X / S}^{\prime}$ be G-invariant Kähler metrics on $T X / S$. Let $\|\cdot\|_{Q}$ and $\|\cdot\|_{Q}^{\prime}$ be the Quillen metrics of $\lambda(X / S, G)$ relative to $g_{X / S}$ and $g_{X / S}^{\prime}$. Then,

$$
\log \left(\frac{\|\cdot\|_{Q}^{\prime}}{\|\cdot\|_{Q}}\right)^{2}=\frac{1}{|G|} \sum_{g \in G} \pi_{*}\left(\widetilde{\operatorname{Td}_{g}}\left(T X / S ; g_{X / S}, g_{X / S}^{\prime}\right)\right)^{(0,0)}
$$


where $\widetilde{\operatorname{Td}_{g}}\left(T X / S ; g_{X / S}, g_{X / S}^{\prime}\right)$ is the Bott-Chern secondary class associated to the $g$-Todd form and $g_{X / S}, g_{X / S}^{\prime}$.

3.2 An Invariant via Analytic Torsion. Let $(X, \iota, \kappa)$ be a 2-elementary $K 3$ surface with $\iota$-invariant Ricci-flat Kähler metric $\kappa$ and symplectic form $\eta_{X}$. The space of $L^{2}-(0, q)$-forms splits into $L^{2}\left(X, \wedge^{0, q}\right)_{ \pm}=\left\{f \in L^{2}\left(X, \wedge^{0, q}\right) ; \iota^{*} f= \pm f\right\}$. Let $\square_{ \pm}^{0, q}$ be the Laplacian restricted to $L^{2}\left(X, \wedge^{0, q}\right)_{ \pm}$. By definition, the RaySinger analytic torsion of $(X / \iota, \kappa)$ is given by $\tau(X / \iota, \kappa)=\operatorname{det} \square_{+}^{0,2} / \operatorname{det} \square_{+}^{0,0}$ because $\zeta_{+}^{0,0}(s)-\zeta_{+}^{0,1}(s)+\zeta_{+}^{0,2}(s) \equiv 0$ where $\zeta_{ \pm}^{0, q}(s)$ is the zeta function of $\square_{ \pm}^{0, q}$. Since $\eta_{X}$ is a parallel section such that $\iota^{*} \eta_{X}=-\eta_{X}$, multiplication by $\eta_{X}$ induces an isometry between $\left(L^{2}\left(X, \wedge^{0,0}\right)_{-}, \Delta_{-}^{0,0}\right)$ and $\left(L^{2}\left(X, \wedge^{0,2}\right)_{+}, \Delta_{+}^{0,2}\right)$. Thus, we get the following.

Lemma 3.1. $\tau(X / \iota, \kappa)=\operatorname{det} \square_{-}^{0,0} / \operatorname{det} \square_{+}^{0,0}$.

Let $p:(X, \iota) \rightarrow S$ be a family of 2-elementary $K 3$ surfaces of type $M$ and $p: Z \rightarrow S$ be the fixed locus. By Theorem 2.5, $Z$ decomposes into $Z=\sum_{i} C_{i}$ where $p: C_{i} \rightarrow S$ is a family of curves over $S$. Let $g_{X / S}$ be a family of Ricci-flat Kähler metrics such that $\iota^{*} g_{X / S}=g_{X / S}$. Set $g_{C_{i} / S}:=\left.g_{X / S}\right|_{C_{i} / S}$ for the induced metric on $T C_{i} / S$ and $g_{N_{C_{i} / X}}$ for the induced metric on the relative normal bundle $N_{C_{i} / X}$ which is defined by the following exact sequence;

$$
0 \longrightarrow T C_{i} / S \longrightarrow T X /\left.S\right|_{C_{i}} \longrightarrow N_{C_{i} / X} \longrightarrow 0
$$

We define four smooth functions over $S$ as follows;

$$
\begin{aligned}
& \tau\left(X / \iota / S, g_{X / S}\right)(s):=\tau\left(X_{s} / \iota, g_{s}\right), \quad \tau\left(C_{i} / S, g_{C_{i} / S}\right)(s):=\tau\left(C_{i, s}, g_{C_{i, s}}\right), \\
& \operatorname{vol}\left(X / S, g_{X / S}\right)(s):=\operatorname{vol}\left(X_{s}, g_{s}\right), \quad \operatorname{vol}\left(C_{i} / S\right)(s):=\operatorname{vol}\left(C_{i, s}, g_{C_{i, s}}\right) .
\end{aligned}
$$

By construction, the following is clear.

\section{Lemma 3.2.}

$$
\left.c_{1}\left(X / S, g_{X / S}\right)\right|_{C_{i} / S}=c_{1}\left(C_{i} / S, g_{C_{i} / S}\right)+c_{1}\left(N_{C_{i} / S}, g_{N_{C_{i} / S}}\right) .
$$

\section{Proposition 3.1.}

$$
\left[\operatorname{Td}_{\iota}\left(X / S, g_{X / S}\right)\right]^{(2,2)}=\sum_{i}\left\{\frac{1}{8} c_{1}\left(X / S, g_{X / S}\right) c_{1}\left(C_{i} / S, g_{C_{i} / S}\right)-\frac{1}{12} c_{1}\left(C_{i} / S, g_{C_{i} / S}\right)^{2}\right\}
$$

Proof. Put $x=c_{1}\left(C_{i} / S, g_{C_{i} / S}\right)$ and $y=c_{1}\left(X / S, g_{X / S}\right)-c_{1}\left(C_{i} / S, g_{C_{i} / S}\right)$. By definition, $(\mathrm{Td} / \mathrm{e})(y+\pi i)=(1+\exp (-y))^{-1}$. Since

$$
\operatorname{Td}(x)=1+\frac{x}{2}+\frac{x^{2}}{12}+O\left(x^{3}\right), \quad \frac{\mathrm{Td}}{\mathrm{e}}(y+\pi i)=\frac{1}{2}+\frac{y}{4}+O\left(y^{3}\right),
$$

we get the assertion by the definition of equivariant Todd form in Theorem 3.1 together with (3.3).

Let $\eta_{t}$ be a symplectic form on $X_{t}$ depending holomorphically in $t \in S$ and $\kappa_{t}$ be a Ricci-flat Kähler metric on $X_{t}$ depending smoothly in $t$. Put $\left\|\eta_{t}\right\|^{2}=\int_{X_{t}} \eta_{t} \wedge \bar{\eta}_{t}$. 


\section{Lemma 3.3.}

$$
\kappa_{t}^{2}=f(t) \eta_{t} \wedge \bar{\eta}_{t}, \quad f(t)=\operatorname{vol}\left(X_{t}, \kappa_{t}\right) /\left\|\eta_{t}\right\|^{2}
$$

Proof. Since $\kappa_{t}$ is Ricci-flat, there exists a constant $C_{t}$ such that $\kappa_{t}^{2}=C_{t} \eta_{t} \wedge \bar{\eta}_{t}$. Integrating both hand sides, we get the assertion.

Let $\Omega_{M}$ be the period domain of 2-elementary $K 3$ surfaces of type $M$. Let $\omega_{M}$ be its Bergman metric and $K_{M}(z, \bar{z})$ its Bergman kernel function;

$$
\omega_{M}(z)=\frac{i}{2 \pi} \partial \bar{\partial} \log K_{M}(z, \bar{z}), \quad K_{M}(z, \bar{z})=\frac{\langle z, \bar{z}\rangle_{N}}{\left|\left\langle z, l_{M}\right\rangle_{N}\right|^{2}}
$$

where $l_{M} \in N_{\mathbb{C}}$ is a vector such that $H_{l_{M}}$ is the hyperplane at infinity of $\mathbb{P}\left(N_{\mathbb{C}}\right)$. Let $\pi: S \rightarrow \Omega_{M}$ be the period map. Since $\pi^{*} \omega_{M}=\frac{i}{2 \pi} \bar{\partial}_{M} \partial_{M} \log \left\|\eta_{t}\right\|^{2}$ by Schumacher's formula ([Sch], [Ti], [To2]), we get the following by taking $\frac{i}{2 \pi} \partial_{M} \bar{\partial}_{M}$ of Lemma 3.3.

\section{Proposition 3.2.}

$$
c_{1}\left(X / S, g_{X / S}\right)=-p^{*}\left\{\pi^{*} \omega_{M}+\frac{i}{2 \pi} \bar{\partial}_{M} \partial_{M} \log \operatorname{vol}\left(X / S, g_{X / S}\right)\right\}
$$

\section{Proposition 3.3.}

$$
\begin{aligned}
& \frac{1}{2}\left[p_{*} \operatorname{Td}\left(X / S, g_{X / S}\right)+p_{*} \operatorname{Td}_{\iota}\left(X / S, g_{X / S}\right)\right]^{(1,1)} \\
& =\frac{r(M)-6}{8}\left\{-\pi^{*} \omega_{M}+\frac{i}{2 \pi} \bar{\partial} \partial \log \operatorname{vol}(X / S)\right\}-\frac{1}{2} \sum_{i} p_{*} \operatorname{Td}\left(C_{i} / S, g_{C_{i} / S}\right)^{(1,1)} .
\end{aligned}
$$

Proof. Since $\chi\left(X_{t}\right)=24$, it follows from Proposition 3.2 and the projection formula that

$$
\begin{aligned}
p_{*} \operatorname{Td}\left(X / S, g_{X / S}\right)^{(1,1)} & =\frac{1}{24}\left[p_{*} c_{1}\left(X / S, g_{X / S}\right) c_{2}\left(X / S, g_{X / S}\right)\right]^{(1,1)} \\
& =-\pi^{*} \omega_{M}+\frac{i}{2 \pi} \bar{\partial}_{M} \partial_{M} \log \operatorname{vol}(X / S)
\end{aligned}
$$

As $\int_{C_{i, t}} c_{1}\left(C_{i, t}\right)=1-g\left(C_{i, t}\right)$, we get by Proposition 3.1 and the projection formula,

$$
\begin{aligned}
& p_{*} \operatorname{Td}_{\iota}\left(X / S, g_{X / S}\right)^{(1,1)} \\
& =p_{*}\left[\sum_{i} \frac{1}{8} c_{1}\left(C_{i} / S, g_{C_{i} / S}\right) p^{*}\left(-\pi^{*} \omega_{M}+\frac{i}{2 \pi} \bar{\partial}_{M} \partial_{M} \log \operatorname{vol}(X / S)\right)-\frac{1}{12} c_{1}\left(C_{i} / S, g_{C_{i} / S}\right)^{2}\right] \\
& =\sum_{i} \frac{1-g\left(C_{i}\right)}{8}\left\{-\pi^{*} \omega_{M}+\frac{i}{2 \pi} \bar{\partial}_{M} \partial_{M} \log \operatorname{vol}(X / S)\right\}-\sum_{i} p_{*} \operatorname{Td}\left(C_{i} / S, g_{C_{i} / S}\right)^{(1,1)} .
\end{aligned}
$$

It follows from Theorem 2.4 that $1 / 2+\sum_{i}\left(1-g\left(C_{i}\right)\right) / 8=(r(M)-6) / 8$ for any $M$ which, together with (3.5-6), yields the assertion. 
Definition 3.3. For a 2-elementary $K 3$ surface of type $M$ with an $\iota$-invariant Ricci-flat Kähler metric $\kappa$, we define $\tau_{M}$ by

$$
\tau_{M}(X, \iota, \kappa):=\langle\kappa, \kappa\rangle^{\frac{14-r(M)}{8}} \tau(X / \iota, \kappa) \prod_{i}\left\langle C_{i}, \kappa\right\rangle^{\frac{1}{2}} \tau\left(C_{i},\left.\kappa\right|_{C_{i}}\right)^{\frac{1}{2}}
$$

Let $\tau_{M / S}$ be the function defined by $\tau_{M / S}(s)=\tau_{M}\left(X_{s}, \iota_{s}, g_{X_{s}}\right)$. Let $j_{C_{i} / S}: S \ni$ $s \rightarrow\left[\operatorname{Jac}\left(C_{i, s}\right)\right] \in \mathcal{A}_{g\left(C_{i}\right)}$ be the period map. Let $\omega_{\mathcal{A}_{g}}$ be the Bergman metric of $\mathcal{A}_{g}$ and $K_{\mathcal{A}_{g}}(\tau)$ the Bergman kernel of $\mathcal{A}_{g}$;

$$
\omega_{\mathcal{A}_{g}}(\tau)=\frac{i}{2 \pi} \bar{\partial}_{\tau} \partial_{\tau} \log K_{\mathcal{A}_{g}}(\tau), \quad K_{\mathcal{A}_{g}}(\tau)=\operatorname{det} \operatorname{Im} \tau
$$

Theorem 3.3.

$$
\frac{i}{2 \pi} \bar{\partial}_{S} \partial_{S} \log \tau_{M / S}=-\frac{r(M)-6}{8} \pi^{*} \omega_{M}-\frac{1}{2} \sum_{i} j_{C_{i} / S}^{*} \omega_{\mathcal{A}_{g(M)}} .
$$

Proof. Consider the family $p: X \rightarrow S$. Put $G=\{1, \iota\}$. Since $H^{q}\left(X_{s}, \mathcal{O}_{X_{s}}\right)^{G}=0$ for $q>0$, it follows that $\lambda(X / S, \iota)=\mathcal{O}_{S} 1_{S}$ where $1_{S}$ is the canonical section of $R_{G}^{0} p_{*} \mathcal{O}_{X}$ such that $1_{S}(s)=1$ in $H^{0}\left(X_{s}, \mathcal{O}_{X_{s}}\right)^{G}$. Since the squared Quillen norm of $1_{S}$ is $\tau(X / \iota / S) \operatorname{vol}(X / S)$, it follows from Theorem 3.1 and Proposition 3.3 that

$$
\begin{aligned}
& \frac{i}{2 \pi} \bar{\partial} \partial \log \tau(X / \iota / S) \operatorname{vol}(X / S) \\
& =\frac{r(M)-6}{8}\left\{\pi^{*} \omega_{M}+\frac{i}{2 \pi} \bar{\partial} \partial \log \operatorname{vol}(X / S)\right\}-\frac{1}{2} \sum_{i} p_{*} \operatorname{Td}\left(C_{i} / S, g_{C_{i} / S}\right)^{(1,1)}
\end{aligned}
$$

Fix a marking of the family of curves $p: C_{i} \rightarrow S$. Let $T\left(\operatorname{Jac}\left(C_{i, s}\right)\right) \in \mathfrak{S}_{g\left(C_{i}\right)}$ be the period matrix of $\operatorname{Jac}\left(C_{i, s}\right)$ relative to this marking. Let $T\left(C_{i} / S\right)$ be a function with values in $\mathfrak{S}_{g\left(C_{i}\right)}$ defined by $T\left(C_{i} / S\right)(s)=T\left(\operatorname{Jac}\left(C_{i, s}\right)\right)$. Theorem 3.1 applied to $p: C_{i} \rightarrow S$ yields

$$
\frac{i}{2 \pi} \bar{\partial} \partial \log \left\{\tau\left(C_{i} / S\right) \operatorname{vol}\left(C_{i} / S\right) \operatorname{det} \operatorname{Im} T\left(J\left(C_{i} / S\right)\right)\right\}=p_{*} \operatorname{Td}\left(C_{i} / S, g_{C_{i} / S}\right)^{(1,1)}
$$

which, together with (3.8) and $\frac{i}{2 \pi} \bar{\partial} \partial \log \operatorname{det} \operatorname{Im} T\left(C_{i} / S\right)=j^{*} \omega_{\mathcal{A}_{g\left(C_{i}\right)}}$, proves the assertion.

Theorem 3.4. $\tau_{M}(X, \iota, \kappa)$ does not depend on $\kappa$, and becomes an invariant of a 2-elementary $K 3$ surface $(X, \iota)$.

Proof. Let $\kappa_{0}$ and $\kappa_{1}$ be two $\iota$-invariant Ricci-flat Kähler metrics of $(X, \iota)$, and set $T(X, \iota, \kappa):=\operatorname{vol}(X, \kappa)^{(r(M)-6) / 8} \tau_{M}(X, \iota, \kappa)$. Let $1_{X} \in H^{0}\left(X, \mathcal{O}_{X}\right)=\lambda(X, \iota)$, $1_{C_{i}} \in H^{0}\left(C_{i}, \mathcal{O}_{C_{i}}\right)$ and $\sigma_{i} \in \operatorname{det} H^{0}\left(C_{i}, \Omega_{C_{i}}^{1}\right)$ be the generators of each line. Let $\|\cdot\|_{Q, \kappa_{j}}$ be the Quillen metric relative to $\kappa_{j}$. Since the $L^{2}$-metric on $\operatorname{det} H^{0}\left(C_{i}, \Omega_{C_{i}}^{1}\right)$ is independent of a choice of Kähler metric, it follows from Theorem 3.2 that

$$
\begin{aligned}
& \log \frac{T\left(X, \iota, \kappa_{0}\right)}{T\left(X, \iota, \kappa_{1}\right)}=\log \frac{\left\|1_{X}\right\|_{Q, \kappa_{0}}^{2}}{\left\|1_{X}\right\|_{Q, \kappa_{1}}^{2}}+\frac{1}{2} \sum \log \frac{\left\|1_{C_{i}} \otimes \sigma_{i}\right\|_{Q, \kappa_{0}}^{2}}{\left\|1_{C_{i}} \otimes \sigma_{i}\right\|_{Q, \kappa_{1}}^{2}} \\
& =\frac{1}{2} \int_{X} \widetilde{\operatorname{Td}}\left(X ; \kappa_{0}, \kappa_{1}\right)+\frac{1}{2} \sum \int_{C_{i}} \widetilde{\operatorname{Td}_{\iota}}\left(X ; \kappa_{0}, \kappa_{1}\right)+\sum \int_{C_{i}} \widetilde{\operatorname{Td}}\left(C_{i} ; \kappa_{0}, \kappa_{1}\right) .
\end{aligned}
$$


Since $\widetilde{\operatorname{Td}}(X)=c_{1}\left(\widetilde{X) c_{2}}(X) / 24, \widetilde{\operatorname{Td}_{\iota}}(X)=\sum_{i} c_{1}\left(\widetilde{X) c_{2}}\left(C_{i}\right) / 8-\widetilde{c_{1}\left(C_{i}\right)^{2}} / 12\right.\right.$, and $\widetilde{\operatorname{Td}}\left(C_{i}\right)=\widetilde{c_{1}\left(C_{i}\right)^{2}} / 12$, we get

$$
\log \frac{T\left(X, \iota, \kappa_{0}\right)}{T\left(X, \iota, \kappa_{1}\right)}=\frac{1}{48} \int_{X} \widetilde{c_{1} c_{2}}(X)\left(\kappa_{0}, \kappa_{1}\right)+\frac{1}{16} \sum \int_{C_{i}} c_{1}\left(\widetilde{X) c_{1}}\left(C_{i}\right)\left(\kappa_{0}, \kappa_{1}\right)\right.
$$

By Yau's theorem ([Ya]), there exists a family of Ricci-flat Kähler metrics $\kappa_{t}(0 \leq$ $t \leq 1)$ joining $\kappa_{0}$ and $\kappa_{1}$. Let $\eta$ be a fixed symplectic form on $X$. As in Lemma 3.3, put $f(t)=\operatorname{vol}\left(X, \kappa_{t}\right) /\|\eta\|^{2}$. Let $L_{t}=\kappa_{t}^{-1} \dot{\kappa}_{t}$ be a section of $\operatorname{End}(T X)$ such that $\kappa_{t}\left(L_{t} u, v\right)=\dot{\kappa}_{t}(u, v)$. By Lemma 3.3, we get $\operatorname{Tr} \kappa_{t}^{-1} \dot{\kappa}_{t}=\partial_{t} \log f(t)$ which, together with the Bott-Chern formula ([B-C], [B-G-S, I, e)]), yields

$$
\begin{aligned}
\int_{X} \widetilde{c_{1} c_{2}}(X)\left(\kappa_{0}, \kappa_{1}\right) & =\left.\int_{X} \int_{1}^{0} d t \frac{d}{d \epsilon}\right|_{\epsilon=0} c_{1}\left(R_{t}+\epsilon \kappa_{t}^{-1} \dot{\kappa}_{t}\right) c_{2}\left(R_{t}+\epsilon \kappa_{t}^{-1} \dot{\kappa}_{t}\right) \\
& =\int_{1}^{0} d t \frac{d}{d t} \log f(t) \int_{X} c_{2}\left(R_{t}\right)=24 \log \frac{\operatorname{vol}\left(X, \kappa_{0}\right)}{\operatorname{vol}\left(X, \kappa_{1}\right)},
\end{aligned}
$$

$$
\begin{aligned}
\int_{C_{i}} c_{1}\left(\widehat{X) c_{1}}\left(C_{i}\right)\left(\kappa_{0}, \kappa_{1}\right)\right. & =\left.\int_{C_{i}} \int_{1}^{0} d t \frac{d}{d \epsilon}\right|_{\epsilon=0} c_{1}\left(R_{t}+\epsilon \kappa_{t}^{-1} \dot{\kappa}_{t}\right) c_{1}\left(R\left(C_{i}\right)+\epsilon \frac{\left.\dot{\kappa}_{t}\right|_{C_{i}}}{\kappa_{t} \mid C_{i}}\right) \\
& =\int_{1}^{0} d t \frac{d}{d t} \log f(t) \int_{C_{i}} c_{1}\left(R_{t}\left(C_{i}\right)\right)=\chi\left(C_{i}\right) \log \frac{\operatorname{vol}\left(X, \kappa_{0}\right)}{\operatorname{vol}\left(X, \kappa_{1}\right)}
\end{aligned}
$$

where $R_{t}$ is the curvature of $\left(X, \kappa_{t}\right)$ and $R_{t}\left(C_{i}\right)$ of $\left(C_{i},\left.\kappa_{t}\right|_{C_{i}}\right)$. By (3.11-13), we get

$$
\log \frac{T\left(X, \iota, \kappa_{0}\right)}{T\left(X, \iota, \kappa_{1}\right)}=\frac{r(M)-6}{8} \log \frac{\operatorname{vol}\left(X, \kappa_{0}\right)}{\operatorname{vol}\left(X, \kappa_{1}\right)}
$$

which, together with the definition of $T(X, \iota, \kappa)$, yields the assertion.

Let $\tau_{M}$ be the function on $\tilde{\Omega}_{M}^{0}$ defined by $\tau_{M}(X, \phi, \iota):=\tau_{M}(X, \iota)$. By Theorems 3.3 and $3.4, \tau_{M}$ is a smooth $\Gamma(M)$-invariant function on $\tilde{\Omega}_{M}^{0}$, and thus descends to a $\Gamma_{M}$-invariant smooth function (denoted by the same symbol $\tau_{M}$ ) on $\Omega_{M}^{0}$ by Theorem 2.4. Applying Theorem 3.3 to the universal family, we get the following.

Theorem 3.5. $\tau_{M}$ descends to a smooth $\Gamma_{M}$-invariant function on $\Omega_{M}^{0}$ and satisfies the following variational formula:

$$
\frac{i}{2 \pi} \bar{\partial} \partial \log \tau_{M}=-\frac{r(M)-6}{8} \omega_{M}-\frac{1}{2} j_{M}^{*} \omega_{\mathcal{A}_{g(M)}}
$$




\section{$\S 4$. Degeneration of $K 3$ Surfaces and Monge-Ampère Equation}

4.1 Apriori Estimates for the Monge-Ampère Equation. Let $(X, \kappa)$ be a compact Kähler surface. Let $F \in C^{\infty}(X)$ be a given function and $\varphi \in C^{\infty}(X)$ satisfies the following complex Monge-Ampère equation;

$$
\left(\kappa+\frac{i}{2 \pi} \partial \bar{\partial} \varphi\right)^{2}=e^{F} \kappa^{2}, \quad \int_{X} \varphi \kappa^{2}=0, \quad \kappa+\frac{i}{2 \pi} \partial \bar{\partial} \varphi>0 .
$$

Put $\kappa^{\prime}:=\kappa+\frac{i}{2 \pi} \partial \bar{\partial} \varphi$ for a new Kähler metric. Let $\Delta$ (resp. $\Delta^{\prime}$ ) be the Laplacian relative to $\kappa$ (resp. $\left.\kappa^{\prime}\right)$. Let $R=\left(R_{i \bar{j} k \bar{l}}\right)$ be the holomorphic bisectional curvature of $(X, \kappa)$. Let $V$ be the volume of $(X, \kappa), \lambda>0$ the first eigen value of $\Delta$, and $S$ the Sobolev constant of $(X, \kappa) ;\|f\|_{L^{4}} \leq S\left(\|d f\|_{L^{2}}+\|f\|_{L^{2}}\right)\left(\forall f \in C^{\infty}(X)\right)$. For $f \in C^{0}(X)$, we denote by $|f|_{\infty}$ the sup-norm.

\section{Proposition 4.1.}

(1) If $|F|_{\infty} \leq 1,|\varphi|_{\infty} \leq C\left(S, \lambda^{-1}, V\right)|F|_{\infty}$ where $C(x, y, z)$ is bounded if all of $x, y, z$ are bounded.

(2) There exist $C_{i}=C_{i}\left(|\Delta F|_{\infty} /|R|_{\infty},|F|_{\infty},|R|_{\infty}|F|_{\infty}, S, \lambda^{-1}, V\right)(i=1,2)$ such that $C_{i}(x, y, z, w, s, t)$ is bounded from above and below if all of $x, y, z, w, s, t$ are bounded, and the following inequality holds on $X ; C_{1} \kappa \leq \kappa^{\prime} \leq C_{2} \kappa$.

Proof. See [Ko, pp.298-299] for (1) and [Ya, pp.350-351, pp.359 1.22-28], [Ko, pp.300-302] for (2).

Proposition 4.2. Let $\varphi \in C^{\infty}(\mathbb{B}(2 r))$ satisfies the Monge-Ampère equation; $\operatorname{det}\left(g_{i \bar{j}}+\frac{i}{2 \pi} \varphi_{i \bar{j}}\right)=e^{F} \operatorname{det}\left(g_{i \bar{j}}\right), \varphi_{i \bar{j}}=\partial^{2} \varphi / \partial z_{i} \partial \bar{z}_{j}$ on the ball of radius $2 r$ in $\mathbb{C}^{2}$, and suppose $\lambda\left(\delta_{i j}\right) \leq\left(g_{i \bar{j}}+\frac{i}{2 \pi} \varphi_{i \bar{j}}\right) \leq \Lambda\left(\delta_{i j}\right)$ over $\mathbb{B}(2 r)$. Then, there exist constants $\alpha=\alpha(\lambda, \Lambda, r)>0$ and $C=C\left(\lambda, \Lambda, r,|\partial \bar{\partial} \varphi|_{C^{0}(\mathbb{B}(2 r))},|F|_{C^{2}(\mathbb{B}(2 r))},\left|g_{i j}\right|_{C^{2}(\mathbb{B}(2 r))}\right) \geq 0$ such that $|\varphi|_{C^{2+\alpha}(\mathbb{B}(r))} \leq C$.

Proof. See [Si, Chap.2, §4].

4.2 Construction of Approximate Ricci-Flat Metrics. Let $\pi: \mathcal{Y} \rightarrow D$ be the degenerating family of $K 3$ surfaces over the disc $D$ considered in Proposition 2.1 whose fiber is denoted by $Y_{t}$. By Proposition 2.1 (5), there exists a coordinate neighborhood $(V, o)$ in $\mathcal{Y}$ such that

$$
(V, o) \cong\left\{(x, y, z, t) \in \mathbb{B}(2) ; x y-z^{2}-t^{2}=0\right\}, \quad \pi(x, y, z, t)=t
$$

where $\mathbb{B}(r)$ is the ball of radius $r$ centered at 0 . Let $L$ be a very ample line bundle over $\mathcal{Y}$ as in Lemma 2.4 and put $L_{t}:=\left.L\right|_{Y_{t}}$. Since $L$ is very ample, we may assume that $\mathcal{Y}$ is a closed subvariety of $\mathbb{P}^{N} \times D$ with $\pi=p r_{2} \circ i$ and $L=\left.\mathcal{O}_{\mathbb{P}^{N}}(1)\right|_{\mathcal{Y}}$. Let $H$ be a hyperplane of $\mathbb{P}^{N}$ such that $i(o) \notin H$. Let $\sigma$ be the section of $L$ such that $(\sigma)_{0}=H \cap \mathcal{Y}$. Let $h_{L}$ be a Hermitian metric of $L$ with the following properties; (P2) there exist open subsets $W^{\prime} \Subset W \Subset V$ such that $h_{L}(\sigma, \sigma) \equiv 1$ on $W^{\prime}$, and $c_{1}\left(L, h_{L}\right):=-\frac{i}{2 \pi} \partial \bar{\partial} \log h_{L}(\sigma, \sigma)=\omega_{F S}$ on $X \backslash W$ where $\omega_{F S}$ is the restriction of the Fubini-Study form of $\mathbb{P}^{N}$.

Let $\kappa_{Y_{t}}$ be the Ricci-flat Kähler metric on $Y_{t}$ cohomologous to $c_{1}\left(L_{t}\right)$. By Kobayashi-Todorov $([\mathrm{K}-\mathrm{T}]), \kappa_{Y_{0}}$ is a Ricci-flat Kähler metric on $Y_{0}$ in the sense 
of orbifold. Let $\eta_{Y_{t}} \in H^{0}\left(Y_{t}, \Omega^{2}\right)$ be a symplectic form on $Y_{t}$ depending holomorphically in $t \in D$. By a suitable choice of $\eta_{Y_{t}}$, we may assume the following;

$$
\kappa_{Y_{t}}^{2}=h(t) \eta_{Y_{t}} \wedge \bar{\eta}_{Y_{t}}, \quad h(t)=\operatorname{deg} L /\left\|\eta_{Y_{t}}\right\|^{2}, \quad h(0)=1
$$

Fix an isomorphism $j:\left(\mathbb{C}^{2} / \pm 1 \cap \mathbb{B}(2), 0\right) \rightarrow\left(Y_{0} \cap V, o\right)$;

$$
j\left(z_{1}, z_{2}\right)=\left(z_{1}^{2}, z_{2}^{2}, z_{1} z_{2}\right) \in Y_{0}
$$

We denote by $(r, \sigma)$ the polar coordinates of $\mathbb{C}^{2} ; r=\|z\|$ and $\sigma=z /\|z\| \in S^{3}$. By [K-T, Theorem 1], we get the following.

Lemma 4.1. There exist $u_{0} \in C^{\infty}\left(Y_{0}\right), v \in C^{\omega}(\mathbb{B}(2))$ and $c>0$ such that $\kappa_{0}=c_{1}\left(L_{0}, e^{-u_{0}} h_{L}\right)$ and $j^{*} u_{0}(z)=c\left(\|z\|^{2}+v(z)\right)$. Here $v$ has the expansion; $v(z)=\sum_{k=2}^{\infty} a_{2 k}(\sigma) r^{2 k}=\sum a_{I J} z^{I} \bar{z}^{J}$ where $a_{2 k}(\sigma)=r^{-2 k} \sum_{|I|+|J|=2 k} a_{I J} z^{I} \bar{z}^{J}$.

For simplicity, we assume $c=1$ in the sequel.( General case is easily obtained by small modifications of this case.) Let $D(\delta)$ be the disc of radius $\delta(\ll 1)$ and fix a smooth trivialization over $D(\delta) ; I:\left(Y_{0} \backslash W\right) \times D(\delta) \cong \mathcal{Y} \backslash W$ such that $i_{t}:=I(\cdot, t)$ : $Y_{0} \backslash W \cong Y_{t} \backslash W$ is a diffeomorphism for any $t$. Set

$$
u_{t}:=\left(i_{t}^{-1}\right)^{*} u_{0} \in C^{\infty}\left(Y_{t} \backslash W\right), \quad h_{t}:=e^{-u_{t}} h_{L} .
$$

Then, $c_{1}\left(L_{t}, h_{t}\right)$ is a $(1,1)$-form on $Y_{t} \backslash W$ approximating $\kappa_{0}$.

Proposition 4.3. There exist $\epsilon, C>0$ with the following properties. If $\delta>0$ is chosen small enough, then for any $|t|<\delta$, one has

(1) $c_{1}\left(L_{t}, h_{t}\right) \geq \epsilon c_{1}\left(L_{t}, h_{L}\right)$ on $Y_{t} \backslash W$,

(2) $\left|\star_{0} i_{t}^{*} c_{1}\left(L_{t}, h_{t}\right)^{2}-1\right| \leq C|t|$ on $Y_{0} \backslash W$.

Here, $\star_{t}$ denotes the Hodge $*$-operator relative to $c_{1}\left(L_{t}, h_{L}\right)=\omega_{F S}$.

Proof. By construction, there exist $\alpha, \beta, \gamma \in C^{\infty}\left(D(\delta) \times\left(Y_{0} \backslash W\right)\right)$ such that

$$
i_{t}^{*} \star_{t} c_{1}\left(L_{t}, h_{t}\right) \wedge c_{1}\left(L_{t}, h_{L}\right)=\alpha, \quad i_{t}^{*} \star_{t} c_{1}\left(L_{t}, h_{t}\right)^{2}=\beta, \quad \star_{0} i_{t}^{*} c_{1}\left(L_{t}, h_{t}\right)^{2}=\gamma
$$

By definition, we get $\alpha(z, 0) \geq 3 \epsilon_{0}, \beta(z, 0) \geq 9 \epsilon_{0}^{2}$ and $\gamma(z, 0)=1$. As $\alpha, \beta, \gamma$ are continuous in $t$, if $|t| \ll 1$, we get $\alpha(t, z) \geq 2 \epsilon_{0}, \beta(t, z) \geq \epsilon_{0}^{2}$ and $|\gamma(t, z)-1| \leq C|t|$, from which the assertion follows.

Remark. In the sequel of this section, we denote by $C(>0)$ a constant independent of $s \in[0,1], t \in D$, and $x \in Y_{t}$, though its value may change in each estimate.

Our next task is to construct an approximating family of Kähler metrics on $W$. Put $\mathcal{X}:=\left\{(x, y, z, t) \in \mathbb{C}^{4} ; x y-z^{2}-t^{2}=0\right\}$ and $\pi(x, y, z, t)=t$. The following is due to Kronheimer $([\mathrm{Kr}])$.

Proposition 4.4. There exists $q \in C^{0}(\mathcal{X}) \cap C^{\infty}(\mathcal{X} \backslash\{0\})$ such that (1) $j^{*} q_{0}=\partial \bar{\partial}\|z\|^{2}$ where $j: \mathbb{C}^{2} / \pm 1 \rightarrow \mathcal{X}_{0}$ is the map defined by (4.4),

(2) $\partial \bar{\partial} q_{t}$ is a Ricci-flat ALE metric on $\mathcal{X}_{t}$ where $q_{t}:=\left.q\right|_{\mathcal{X}_{t}}, \mathcal{X}_{t}:=\pi^{-1}(t)$

(3) $q$ is homogeneous of order $1 ; q(s x, s y, s z, s t)=|s| q(x, y, z, t)$.

Remark. A Riemannian manifold $(M, g)$ is ALE if there exists a compact subset $K$ of $M$, a finite group $\Gamma \subset O(m, \mathbb{R})$ and a diffeomorphism $f: \mathbb{R}^{m} \backslash \mathbb{B}(R) / \Gamma \rightarrow M \backslash K$ such that $f^{*} g=\delta+O\left(r^{-2-k}\right)$ for some $k>0$ where $\delta$ is the Euclidean metric. 
By (4.3), we identify a neighborhood $V$ of $o$ in $\mathcal{Y}$ with a neighborhood around 0 of $\mathcal{X}$. Thus $Y_{t} \cap V=\mathcal{X}_{t} \cap \mathbb{B}(2)$ and we regard $q_{t}$ to be a function on $Y_{t} \cap V$. Put $S:=\{x \in \mathcal{X} ; q(x)=1\}$ and $S_{t}:=S \cap \mathcal{X}_{t}$ for the level set of $q$, and $S_{<\delta}:=\bigcup_{|t|<\delta} S_{t}$ for the sublevel set. We consider $S_{t}$ to be a subset in $\mathbb{C}^{3}$. As $\pi: S_{<\delta} \rightarrow D(\delta)$ is of maximal rank when $\delta \ll 1$, we can construct a trivialization of $S_{<\delta}$ by integrating vector fields $\xi, \zeta$ on $S_{<\delta}$ such that $\pi_{*} \xi=\partial / \partial u$ and $\pi_{*} \zeta=\partial / \partial v$ where $t=u+i v$ is the coordinate of $D(\delta)$.

Lemma 4.2. There exists a trivialization $\Psi: S_{0} \times D(\delta) \rightarrow S_{<\delta}$ with the property that $\Psi(\cdot, t): S_{0} \rightarrow S_{t}$ is a diffeomorphism and that $\Psi(\cdot, 0)$ is the identity map on $S_{0}$. We put $\psi_{t}(\cdot):=\Psi(\cdot, t)$ and $\phi_{t}=\psi_{t}^{-1}: S_{t} \rightarrow S_{0}$ for the inverse map.

With this identification of $S_{t}$ with $S_{0}$, we can construct a good deformation of the Kähler potential by using the polar coordinates in Lemma 4.1. Namely, if $u_{0}=q_{0}+\sum_{k>2} a_{2 k}(\sigma) q_{0}^{k}$ is the expansion in the polar coordinates, then

$$
u_{t}=q_{t}+\sum_{k>2}\left(\phi_{t}^{*} a_{2 k}\right) q_{t}^{2 k}
$$

will be a good approximation of $u_{0}$. Let us verify it in the sequel.

Set $Y_{t, a}:=\left\{x \in Y_{t} \cap V ; q_{t}(x) \geq a\right\}$. We extend $\phi_{t}: Y_{t, \delta^{-1}|t|} \rightarrow Y_{0, \delta^{-1}|t|}$ and $\psi_{t}: Y_{0, \delta^{-1}|t|} \rightarrow Y_{t, \delta^{-1}|t|}$ by using the $\mathbb{R}_{+}$-action. Namely, we define

$$
\phi_{t}(x):=q_{t}(x) \cdot \phi_{q_{t}(x)^{-1} t}\left(q_{t}(x)^{-1} x\right), \quad \psi_{t}(x):=q_{0}(x) \cdot \psi_{q_{0}(x)^{-1} t}\left(q_{0}(x)^{-1} x\right)
$$

where $\lambda \cdot x=(\lambda x, \lambda y, \lambda z)$ if $x=(x, y, z) \in \mathbb{C}^{3}$. Put $\mu_{\lambda}(x):=\lambda \cdot x$. Let $J_{t}$ be the complex structure of $Y_{t}$. Let $\bar{\partial}_{Y_{t}}$ be the $\bar{\partial}$-operator of $Y_{t}$. Via $\psi_{t}$, identify $\bar{\partial}_{Y_{t}}$ with the $\bar{\partial}$-operator of $\left(Y_{0, \delta^{-1}|t|} \cap V, \psi_{t}^{*} J_{t}\right)$ and similarly $\partial_{Y_{t}}$ with the $\partial$-operator. We denoted them by $\bar{\partial}_{t}$ and $\partial_{t}$ respectively;

$$
\partial_{t}:=\psi_{t}^{*} \circ \partial_{Y_{t}} \circ \phi_{t}^{*}, \quad \bar{\partial}_{t}:=\psi_{t}^{*} \circ \bar{\partial}_{Y_{t}} \circ \phi_{t}^{*}, \quad \partial_{t} \bar{\partial}_{t}=\psi_{t}^{*} \circ \partial_{Y_{t}} \bar{\partial}_{Y_{t}} \circ \phi_{t}^{*} .
$$

Take $f \in C^{\infty}\left(Y_{0} \cap V\right)$ and identify it with an even function on $\mathbb{C}^{2} \cap \mathbb{B}(2)$ via $j$. Let $\left(z_{1}, z_{2}\right)$ be the complex coordinates of $\mathbb{C}^{2}$, and $z_{1}=x_{1}+i x_{2}, z_{2}=x_{3}+i x_{4}$ be the real coordinates. For $z \in Y_{0, \delta^{-1}|t|} \cap V$, we can write

$$
\partial_{t} \bar{\partial}_{t} f(z)=\sum a_{i j}^{k l}(t, z) \partial_{k l} f(z) d x_{i} \wedge d x_{j}+\sum b_{i j}^{k}(t, z) \partial_{k} f(z) d x_{i} \wedge d x_{j}
$$

where $a_{i j}^{k l}$ and $b_{i j}^{k}$ restricted to $D(\delta) \times S_{0}$ are $C^{\infty}$-functions, and $\partial_{i} f=\partial f / \partial x_{i}$ etc.

Lemma 4.3. Putting $\|z\|=r$, one has the following in the polar coordinates:

$$
\partial_{t} \bar{\partial}_{t} f(z)=\sum\left\{a_{i j}^{k l}\left(r^{-2} t, \sigma\right) \partial_{k l} f(z)+r^{-1} b_{i j}^{k}\left(r^{-2} t, \sigma\right) \partial_{k} f(z)\right\} d x_{i} \wedge d x_{j}
$$

Proof. As $\mu_{\lambda} j(z)=j(\sqrt{\lambda} z)$ by (4.5), the action of $\mathbb{R}_{+}$on $Y_{0} \cap V$ is expressed by $\mu_{\lambda}(z)=\left(\sqrt{\lambda} z_{1}, \sqrt{\lambda} z_{2}\right)$. Since $\phi_{\lambda t} \circ \mu_{\lambda}=\mu_{\lambda} \circ \phi_{t}$ on $\mathbb{R}_{+} \times Y_{t, \delta^{-1}|t|}$ by definition, we get $\partial_{\lambda t} \bar{\partial}_{\lambda t}=\mu_{\lambda^{-1}}^{*} \circ \partial_{t} \bar{\partial}_{t} \circ \mu_{\lambda}^{*}$ which yields $a_{i j}^{k l}\left(\lambda^{-1} t, \lambda^{-1 / 2} z\right)=a_{i j}^{k l}(t, z)$ and $\lambda^{-1 / 2} b_{i j}^{k}\left(\lambda^{-1} t, \lambda^{-1 / 2} z\right)=b_{i j}^{k}(t, z)$. Putting $\lambda=r$, we get the assertion. 
Let $\omega_{Y_{t}}$ be the Kronheimer's Ricci-flat ALE metric on $Y_{t} \cap V$;

$$
\omega_{Y_{t}}:=\partial_{Y_{t}} \bar{\partial}_{Y_{t}} q_{t}
$$

As $\psi_{t}^{*} q_{t}=q_{0}$, we find that $\omega_{t}:=\partial_{t} \bar{\partial}_{t} q_{0}$ is the Kähler form on $\left(Y_{0, \delta^{-1}|t|} \cap V, \psi_{t}^{*} J_{t}\right)$. Taking $f(z)=\|z\|^{2}$ in (4.10), it follows that, for any $z \in Y_{0, \delta^{-1}|t|} \cap V$,

$$
\omega_{t}(z)=\sum g_{i j}\left(r^{-2} t, \sigma\right) d x_{i} \wedge d x_{j}
$$

where $g_{i j}$ is a smooth functions on $D(\delta) \times S_{0}$. Let $\rho$ be a cut-off function such that $\rho(s) \equiv 0$ for $s \leq 2 \delta^{-1}, \rho(s) \equiv 1$ for $s \geq 4 \delta^{-1}, 0 \leq \rho^{\prime}(s) \leq C_{0} \delta$ and $\left|\rho^{\prime \prime}(s)\right| \leq C_{0} \delta^{2}$. Let $v \in C^{\omega}(\mathbb{B}(1))$ be the error term of $u_{0}$ appeared in Lemma 4.1. Set

$$
v_{t}(x):=\rho_{t}(x) \phi_{t}^{*} v(x), \quad \rho_{t}(x):=\rho\left(|t|^{-1} q_{t}(x)\right), \quad \tilde{v}_{t}:=\rho\left(|t|^{-1} r^{2}\right) v=\psi_{t}^{*} v_{t} .
$$

Then, $v_{t}, \rho_{t} \in C^{\infty}\left(Y_{t}\right)$ and $\tilde{v}_{t} \in C^{\infty}\left(Y_{0}\right)$. By (4.8), we get $\psi_{t}^{*} \partial_{Y_{t}} \bar{\partial}_{Y_{t}} v_{t}=\partial_{t} \bar{\partial}_{t} \tilde{v}_{t}$.

Lemma 4.4. Let $*_{t}$ be the Hodge $*$-operator relative to $\omega_{t}$. There exist $B_{i} \in$ $C^{\infty}\left(D(\delta) \times[0,1] \times S_{0}\right)(i=1,2)$ such that, for any $z \in Y_{0, \delta^{-1}|t|} \cap V$,

$$
*_{t}\left(\partial_{t} \bar{\partial}_{t} \tilde{v}_{t} \wedge \omega_{t}\right)(z)=r^{2} B_{1}\left(r^{-2}|t|, r^{2}, \sigma\right), \quad *_{t}\left(\partial_{t} \bar{\partial}_{t} \tilde{v}_{t}\right)^{2}(z)=r^{4} B_{2}\left(r^{-2}|t|, r^{2}, \sigma\right) .
$$

Proof. As $v \in C^{\omega}\left([0,1] \times S^{3}\right)$ by Lemma 4.1, it follows from (4.12) and

$$
\begin{aligned}
r^{-1} \partial_{i}\left\{\rho\left(|t|^{-1} r^{2}\right) v\right\}= & 2|t|^{-1} r^{-1} x_{i} \rho^{\prime}\left(|t|^{-1} r^{2}\right) v+r^{-1} \rho\left(|t|^{-1} r^{2}\right) \partial_{i} v \\
\partial_{i j}\left\{\rho\left(|t|^{-1} r^{2}\right) v\right\}= & 4|t|^{-2} \rho^{\prime \prime}\left(|t|^{-1} r^{2}\right) x_{i} x_{j} v+2|t|^{-1} r^{2} \rho^{\prime}\left(|t|^{-1} r^{2}\right) \delta_{i j} v \\
& +2|t|^{-1} \rho^{\prime}\left(|t|^{-1} r^{2}\right)\left(x_{j} \partial_{i} v+x_{i} \partial_{j} v\right)+\rho\left(|t|^{-1} r^{2}\right) \partial_{i j} v
\end{aligned}
$$

that there exists $A_{i} \in C^{\infty}\left(D(\delta) \times[0,1] \times S_{0}\right)$ such that $\omega_{t}^{2}(z)=A_{0}\left(r^{-2}|t|, r^{2}, \sigma\right) d V$, $\partial_{t} \bar{\partial}_{t} \tilde{v}_{t} \wedge \omega_{t}(z)=r^{2} A_{1}\left(r^{-2}|t|, r^{2}, \sigma\right) d V$ and $\left(\partial_{t} \bar{\partial}_{t} \tilde{v}_{t}\right)^{2}(z)=r^{4} A_{2}\left(r^{-2}|t|, r^{2}, \sigma\right) d V$ where $d V$ is the volume form of $\left(Y_{0}, \omega_{0}\right)$. Since $\omega_{0}^{2}=d V$, we get $A_{0}(0, r, \sigma) \equiv 1$ for any $(r, \sigma) \in[0,1] \times S_{0}$. By the compactness of $[0,1] \times S_{0}$, there exists $C_{0}$ such that $0<C_{0}^{-1} \leq A_{0}(s, r, \sigma) \leq C_{0}<\infty$ for $|s|<\delta$ and $(r, \sigma) \in[0,1] \times S_{0}$. Since $*_{t} F=F / \omega_{t}^{2}$ for a 4 -form $F$, we get $B_{1}=A_{1} / A_{0}$ and $B_{2}=A_{2} / A_{0}$.

We define $\Omega_{Y_{t}}$ and $G_{Y_{t}}$ as follows;

$$
\Omega_{Y_{t}}:=\omega_{Y_{t}}+\partial_{Y_{t}} \bar{\partial}_{Y_{t}} v_{t}, \quad G_{Y_{t}}:=\Omega_{Y_{t}}^{2} / \omega_{Y_{t}}^{2} .
$$

As before, set $\Omega_{t}:=\psi_{t}^{*} \Omega_{Y_{t}}=\omega_{t}+\partial_{t} \bar{\partial}_{t} \tilde{v}_{t}$ and $G_{t}:=\psi_{t}^{*} G_{Y_{t}}$.

Proposition 4.5. If $|t| \ll 1, \Omega_{Y_{t}}$ becomes a Kähler metric on $Y_{t} \cap V$.

Proof. When $q_{t}(x) \leq \delta^{-1}|t|, v_{t} \equiv 0$ by (4.13) and $\Omega_{Y_{t}}>0$ because $\Omega_{Y_{t}}=\omega_{Y_{t}}$ in this case. Thus, it is enough to show that ${ }_{t} \Omega_{t} \wedge \omega_{t}(z)>0$ and $*_{t} \Omega_{t}^{2}(z)>0$ for any $z \in Y_{0, \delta^{-1}|t|} \cap V$ if $|t| \ll 1$. By Lemma 4.4, we get

${ }_{*} \Omega_{t} \wedge \omega_{t}(z)=1+r^{2} B_{1}\left(|t| / r^{2}, r^{2}, \sigma\right),{ }_{t} \Omega_{t}^{2}=1+2 r B_{1}\left(|t| / r^{2}, r^{2}, \sigma\right)+r^{2} B_{2}\left(|t| / r^{2}, r^{2}, \sigma\right)$. 
As $1+r^{2} B_{1}\left(0, r^{2}, \sigma\right) \geq C>0$ for any $z \in \mathbb{B}(1)=Y_{0} \cap V$ because $\Omega_{0}$ is a Kähler metric, choosing $t$ small enough, the right hand side of the first formula of (4.16) is greater than $C / 2$. Similarly, the rest inequality can be shown.

Let $\tau_{Y_{t}}$ be the holomorphic family of symplectic forms on $Y_{t} \cap V$ such that $\tau_{Y_{t}} \wedge \bar{\tau}_{Y_{t}}=\omega_{Y_{t}}^{2}$. Under the identification of $Y_{t} \cap V$ with $\mathcal{X}_{t} \cap \mathbb{B}(2)$, we get $\omega_{Y_{t}}=$ $g_{Y_{t}}\left(I_{t}, \cdot\right)$ and $\tau_{Y_{t}}=g_{Y_{t}}\left(J_{t}, \cdot, \cdot\right)+\sqrt{-1} g_{Y_{t}}\left(K_{t} \cdot, \cdot\right)$ where $g_{Y_{t}}$ is the underlying Riemannian metric and $\left(I_{t}, J_{t}, K_{t}\right)$ are complex structures defining the hyper-Kähler structure. Since $(V, o)$ is normal, there exists a holomorphic function $f_{V} \in \mathcal{O}(V)$ such that $\left.\eta_{Y_{t}}\right|_{V}=f_{Y_{t}} \cdot \tau_{Y_{t}}$ and $f_{Y_{t}}:=\left.f_{V}\right|_{Y_{t}}$. As $f_{Y_{t}}$ has no zero for any $t \in B(\delta)$, $f_{V}$ has no zero on $V$. Thus there exists the lower bound on $V ;\left|f_{V}(x)\right| \geq C>0$. By (4.3), (4.15) and the definition of $f_{Y_{t}}$, we get

$$
\left|f_{Y_{0}}\right|^{2}=\left(\eta_{Y_{0}} \wedge \bar{\eta}_{Y_{0}}\right) /\left(\tau_{Y_{0}} \wedge \bar{\tau}_{Y_{0}}\right)=\Omega_{0}^{2} / \omega_{0}^{2}=G_{0} .
$$

Let $u_{t}$ be the function on $Y_{t} \backslash W$ as in (4.6) and $h_{L}$ be the Hermitian metric of $L$ with the property (P2) as before. Let $\chi \geq 0$ be a cut-off function on $\mathcal{Y}$ such that $\chi(x) \equiv 0$ for $x \in W$ and $\chi(x) \equiv 1$ for $x \in \mathcal{Y} \backslash V$. We shall use the following $(1,1)$-form $\tilde{\kappa}_{Y_{t}}$ as a background metric in approximating $\kappa_{Y_{t}}$;

$\tilde{\kappa}_{Y_{t}}:=c_{1}\left(L_{t}, e^{-\theta_{t}} h_{L}\right)=\frac{i}{2 \pi} \partial_{Y_{t}} \bar{\partial}_{Y_{t}}\left\{\theta_{t}-\log h_{L}(\sigma, \sigma)\right\}, \quad \theta_{t}:=\chi u_{t}+(1-\chi)\left(q_{t}+v_{t}\right)$.

Proposition 4.6. If $|t| \ll 1, \tilde{\kappa}_{Y_{t}}$ is a Kähler metric with the following estimate;

$$
\left|\frac{\eta_{Y_{t}} \wedge \bar{\eta}_{Y_{t}}}{\tilde{\kappa}_{Y_{t}}^{2}}-1\right|_{\infty} \leq C|t|
$$

Proof. (1) Suppose $x=\psi_{t}(z) \in Y_{t, \delta^{-1}|t|} \cap V$. By definition, one has

$$
\eta_{Y_{t}} \wedge \bar{\eta}_{Y_{t}} / \Omega_{Y_{t}}^{2}=\left(\eta_{Y_{t}} \wedge \bar{\eta}_{Y_{t}} / \omega_{Y_{t}}^{2}\right) \cdot\left(\Omega_{Y_{t}}^{2} / \omega_{Y_{t}}^{2}\right)^{-1}=\left|f_{Y_{t}}\right|^{2} G_{Y_{t}}^{-1} .
$$

As $G_{t}(z)=1+r^{2} B\left(\frac{|t|}{r^{2}}, r^{2}, \sigma\right)$ where $B\left(s, r^{2}, \sigma\right):=B_{1}\left(s, r^{2}, \sigma\right)+r^{2} B_{2}\left(s, r^{2}, \sigma\right)$,

$$
\left|G_{t}(z)-G_{0}(z)\right|=r^{2}\left|B\left(s, r^{-2}|t|, \sigma\right)-B(s, 0, \sigma)\right| \leq|d B|_{\infty}|t| \leq C|t| .
$$

Let $\|\cdot\|$ be the Euclidean norm of $\mathbb{C}^{3}$. Then, we get

$$
\left.|| f_{V}\left(\psi_{t}(z)\right)\right|^{2}-\left.\left|f_{Y_{0}}(z)\right|^{2}|\leq| f_{V}\right|_{\infty}\left|d f_{V}\right|_{\infty}\left\|\psi_{t}(j(z))-j(z)\right\| \leq C|t|
$$

which, together with (4.19-20), yields that

$$
\begin{aligned}
\left|\psi_{t}^{*} \frac{\eta_{Y_{t}} \wedge \bar{\eta}_{Y_{t}}}{\Omega_{Y_{t}}^{2}}(z)-1\right|=\left|\frac{\psi_{t}^{*}\left|f_{V}\right|^{2}-G_{t}}{G_{t}}\right| & \leq \frac{\left.\left|\psi_{t}^{*}\right| f_{V}\right|^{2}-\left|f_{Y_{0}}\right|^{2}|+| G_{t}-G_{0} \mid}{\left|G_{t}\right|} \\
& \leq \frac{C|t|}{\left|G_{0}\right|-C|t|}
\end{aligned}
$$

Since both $\Omega_{Y_{0}}$ and $\omega_{Y_{0}}$ are Kähler metrics on $Y_{0} \cap V$, there exists $C>0$ such that $\left|G_{0}\right|-C|t| \geq C$ on $Y_{0} \cap V$, which, together with (4.22), yields the assertion. 
(2) If $q_{t}(x) \leq \delta^{-1}|t|$, it follows from (4.13) and (4.15) that $\Omega_{Y_{t}}(x)=\omega_{Y_{t}}(x)$ and

$$
\eta_{Y_{t}} \wedge \bar{\eta}_{Y_{t}}(x) / \Omega_{Y_{t}}^{2}(x)=\eta_{Y_{t}} \wedge \bar{\eta}_{Y_{t}}(x) / \omega_{Y_{t}}^{2}(x)=\left|f_{Y_{t}}(x)\right|^{2}
$$

By (4.17), we get $f_{Y_{t}}(0)=1$ because $\Omega_{0}(0)=\omega_{0}(0)$ by construction which, together with (4.23), yields

$$
\left|\frac{\eta_{Y_{t}} \wedge \bar{\eta}_{Y_{t}}}{\Omega_{Y_{t}}^{2}}(x)-1\right|=\left.|| f_{V}(x)\right|^{2}-\left.\left|f_{V}(0)\right|^{2}|\leq| f_{V}\right|_{\infty}\left|d f_{V}\right|_{\infty}\|x\| .
$$

By Proposition $4.4(3)$, one has $C^{-1}(\|x\|+|t|) \leq q_{t}(x) \leq C(\|x\|+|t|)$ which, together with $q_{t}(x) \leq \delta^{-1}|t|$, implies $\|x\| \leq C|t|$ and yields the assertion.

(3) Consider $Y_{t} \backslash W$. By construction (Lemma 4.1 and (4.15)), we get $u_{0}=q_{0}+v_{0}$ on $V$. Therefore, $\tilde{\kappa}_{Y_{0}}=\kappa_{Y_{0}}$ and there is a smooth function $w$ on $V \backslash W$ such that $u_{t}-\left.\left(q_{t}+v_{t}\right)\right|_{V \backslash W}=\left.t w\right|_{Y_{t} \cap(V \backslash W)}+\left.\bar{t} \bar{w}\right|_{Y_{t} \cap(V \backslash W)}$. Thus there exist $(1,1)-$ forms $\xi$ and $\xi^{\prime}$ on $V \backslash W$ such that $\left.\tilde{\kappa}_{Y_{t}}\right|_{V \backslash W}=c_{1}\left(L_{t}, h_{t}\right)+t \xi+\bar{t} \xi^{\prime}$ which, together with Proposition 4.4, implies that $\tilde{\kappa}_{Y_{t}}$ is positive on $Y_{t} \cap(V \backslash W)$. As $\tilde{\kappa}_{Y_{t}}>0$ on $\left(Y_{t} \backslash V\right) \cup\left(Y_{t} \cap W\right)$ by Propositions 4.4 and 4.5 , it becomes a Kähler metric on $Y_{t}$. Now, the desired estimate follows from Propositions 4.3 and 4.6.

If $|t| \ll 1$, the following extension of $\omega_{Y_{t}}$ becomes a Kähler metric on $Y_{t}$;

$$
\omega_{Y_{t}}:=c_{1}\left(L_{t}, e^{-w_{t}} h_{L}\right)=\frac{i}{2 \pi} \partial_{Y_{t}} \bar{\partial}_{Y_{t}}\left\{w_{t}-\log h_{L}(\sigma, \sigma)\right\}, \quad w_{t}:=\chi u_{t}+(1-\chi) q_{t} .
$$

By construction, $\left.\omega_{Y_{t}}\right|_{Y_{t} \cap W}=\partial_{Y_{t}} \bar{\partial}_{Y_{t}} q_{t}$. Let $\tilde{\kappa}_{t}(s)(0 \leq s \leq 1)$ be the homotopy of Kähler metrics on $Y_{t}$ joining $\tilde{\kappa}_{Y_{t}}$ and $\omega_{Y_{t}}$ defined by

$$
\tilde{\kappa}_{t}(s):=s \tilde{\kappa}_{Y_{t}}+(1-s) \omega_{Y_{t}}
$$

Proposition 4.7. Let $\operatorname{Ric}\left(\tilde{\kappa}_{t}(s)\right)=\partial_{Y_{t}} \bar{\partial}_{Y_{t}} \log \left(\tilde{\kappa}_{t}(s)^{2} / \eta_{Y_{t}} \wedge \bar{\eta}_{Y_{t}}\right)$ be the Ricci curvature of $\left(Y_{t}, \tilde{\kappa}_{t}(s)\right)$. Then, one has $\left|\operatorname{Ric}\left(\tilde{\kappa}_{t}(s)\right)\right|_{\infty} \leq C$.

Proof. As the assertion is clear for $x \in Y_{t} \backslash W$, we suppose that $x \in Y_{t} \cap W$. When $q_{t}(x) \leq \delta^{-1}|t|, \tilde{\kappa}_{Y_{t}}(s)=\omega_{Y_{t}}$ by construction, which is followed by the assertion as $\omega_{Y_{t}}$ is Ricci-flat. When $x=\psi_{t}(r, \sigma) \in Y_{t, \delta^{-1}} \cap W$, it follows from (4.19) that

$$
\psi_{t}^{*} \operatorname{Ric}\left(\tilde{\kappa}_{t}(s)\right)=\partial_{t} \bar{\partial}_{t} \log \left\{1+r^{2} B^{\prime}\left(s, r^{-2}|t|, r^{2}, \sigma\right)\right\}
$$

because $\partial_{Y_{t}} \bar{\partial}_{Y_{t}} \log \left|f_{Y_{t}}\right|^{2}=0$ where $B^{\prime}\left(s, u, r^{2}, \sigma\right):=2 s B_{1}\left(u, r^{2}, \sigma\right)+s^{2} r^{2} B_{2}\left(u, r^{2}, \sigma\right)$. As $\left|r^{-1} \partial_{i}\left(r^{2} B^{\prime}\right)\right|_{\infty}+\left|\partial_{i j}\left(r^{2} B^{\prime}\right)\right|_{\infty} \leq C$, we get the assertion.

Let $\tilde{R}_{t}(s)$ be the curvature of $\left(Y_{t}, \tilde{\kappa}_{t}(s)\right)$.

Proposition 4.8. For any $(t, x) \in D(\delta) \times Y_{t} \cap W$, one has $\left|\tilde{R}_{t}(s)(x)\right| \leq C q_{t}(x)^{-1}$.

Proof. By (4.10-11) and (4.15), there exists $g_{i j} \in C^{\infty}\left(D(\delta) \times[0,1] \times S_{0} \times[0,1]\right)$ such that $\psi_{t}^{*} \tilde{\omega}_{t}(s)(z)=\sum_{i j} g_{i j}\left(r^{-1}|t|, r^{2}, \sigma, s\right) d x_{i} d x_{j}$ and $\left(g_{i j}\right)$ is an uniformly positive definite matrix. As $\left|\partial^{k} g_{i j}\left(r^{-1}|t|, r^{2}, \sigma, s\right)\right| \leq C_{k} r^{-k}$, we get the assertion. 
Theorem 4.1. For any $(t, s) \in D(\delta) \times[0,1]$, one has $C^{-1} \tilde{\kappa}_{t}(s) \leq \kappa_{Y_{t}} \leq C \tilde{\kappa}_{t}(s)$.

Proof. Since $\tilde{\kappa}_{t}(s)=\omega_{Y_{t}}+s \partial_{Y_{t}} \bar{\partial}_{Y_{t}} v_{t}$ on $Y_{t} \cap V$, one has $C^{-1} \omega_{Y_{t}} \leq \tilde{\kappa}_{t}(s) \leq C \omega_{Y_{t}}$ by (4.16). Thus, it is enough to show the inequality when $s=0$. Consider the Monge-Ampère equation (4.1) with $X=Y_{t}, \kappa=\tilde{\kappa}_{Y_{t}}, \phi=\phi_{t}$ and $F=F_{t}=$ $\log \left(h(t) \eta_{Y_{t}} \wedge \bar{\eta}_{Y_{t}} / \tilde{\kappa}_{Y_{t}}^{2}\right)$. Here $h(t)$ is defined in (4.3). Since $h(t)=1+O(|t|)$ as $t \rightarrow 0$, it follows from Proposition 4.7 that $\left|F_{t}\right|_{\infty} \leq C|t|$. Thus, by Propositions 4.78, all of $\left|\Delta_{t} F_{t}\right|_{\infty} /\left|R_{t}\right|_{\infty},\left|F_{t}\right|_{\infty},\left|R_{t}\right|_{\infty}\left|F_{t}\right|_{\infty}, V_{t}$ are uniformly bounded in $t \in D(\delta)$ where the subscript $t$ means that these quantities are considered relative to $\tilde{\kappa}_{Y_{t}}$. As $\tilde{\kappa}_{Y_{t}}$ is quasi-isometric to $\omega_{Y_{t}}$ on $Y_{t} \cap V$, the Sobolev constant $S_{t}$ is uniformly bounded. As $\lambda_{t}$, the first eigenvalue of the Laplacian of $\left(Y_{t}, \tilde{\kappa}_{Y_{t}}\right)$ is continuous in $t$ ([Yo1, Theorem 5.1]), we also get an uniform bound $\lambda_{t}^{-1}<C$ because $\lambda_{0}>0$. The assertion follows from Proposition 4.1 (2) together with these bounds.

Consider the following Monge-Ampère equation on $Y_{t}$;

$$
\left(\tilde{\kappa}_{Y_{t}}+\frac{i}{2 \pi} \partial \bar{\partial} \varphi_{t}(s)\right)^{2}=e^{s F_{t}+a_{t}(s)} \tilde{\kappa}_{Y_{t}}^{2}, \quad \int_{Y_{t}} \varphi_{t}(s) \tilde{\kappa}_{Y_{t}}^{2}=0
$$

where $F_{t}=\log \left(h(t) \eta_{Y_{t}} \wedge \bar{\eta}_{Y_{t}} / \tilde{\kappa}_{Y_{t}}^{2}\right)$ and $a_{t}(s)=\log \left(\operatorname{deg} L / \int_{Y_{t}} e^{s F_{t}} \tilde{\kappa}_{Y_{t}}^{2}\right)$. Set $\kappa_{t}(s)$ for the smooth homotopy of Kähler metrics joining $\tilde{\kappa}_{Y_{t}}$ and $\kappa_{Y_{t}}$;

$$
\kappa_{t}(s):=\tilde{\kappa}_{Y_{t}}+\frac{i}{2 \pi} \partial \bar{\partial} \varphi_{t}(s)>0, \quad \kappa_{t}(0)=\tilde{\kappa}_{Y_{t}}, \quad \kappa_{t}(1)=\kappa_{Y_{t}}
$$

Theorem 4.2. For any $t \in D, s \in[0,1]$ and $x \in Y_{t}$, one has

$$
\left|F_{t}\right|_{\infty}+\left|a_{t}(s)\right| \leq C|t|, \quad C^{-1} \tilde{\kappa}_{Y_{t}}(x) \leq \kappa_{t}(s)(x) \leq C \tilde{\kappa}_{Y_{t}}(x), \quad\left|\operatorname{Ric}\left(\kappa_{t}(s)\right)\right|_{\infty} \leq C .
$$

Proof. The first inequality follows from Proposition 4.8, the third one from Proposition 4.7 and (4.28). The second one is similarly proved as Theorem 4.1.

Let $R_{t}(s)$ be the curvature of $\left(Y_{t}, \kappa_{t}(s)\right)$ and $\nabla_{t}(s)$ its covariant derivative.

Theorem 4.3. On $Y_{t} \cap W$, one has $\left|\nabla_{t}(s)^{k} R_{t}(s)(x)\right| \leq C q_{t}(x)^{-1-\frac{k}{2}}$.

Proof. For $p \in S_{s}$, let $B(p, r)$ be the metric ball of radius $r$ centered at $p$ relative to the metric $\omega_{Y_{s}}$, and $w=\left(w_{1}, w_{2}\right)$ its holomorphic normal coordinates. Fix $\epsilon>0$ small enough so that $(B(p, \epsilon), w)$ becomes a coordinate neighborhood for any $p \in S_{s}(s \in D(\delta))$. Take $x_{0} \in Y_{t, \delta^{-1}|t|} \cap W$. Put $y_{0}:=q_{t}\left(x_{0}\right)^{-1} x_{0} \in S_{q_{t}\left(x_{0}\right)^{-1} t}$ and $B=B\left(y_{0}, \epsilon\right)$. For $y \in B$, put $x:=q_{t}\left(x_{0}\right) y \in Y_{t}$ which makes $(B, w)$ to be a coordinate neighborhood of $Y_{t}$ at $x_{0}$. As $y=y_{0}+w, v(z)=O\left(\|z\|^{4}\right)$ and $q_{t}\left(x_{0}\right)^{-1}\left(q_{t}(x)+v_{t}(x)\right)=q_{q_{t}\left(x_{0}\right)^{-1} t}(y)+q_{t}\left(x_{0}\right)^{-1} v\left(q_{t}\left(x_{0}\right) \phi_{q_{t}\left(x_{0}\right)^{-1} t}(y)\right)$, we get $\left|q_{t}\left(x_{0}\right)^{-1}\left(q_{t}+v_{t}\right)\right|_{C^{k}(B)} \leq C\left(k,|v|_{C^{k}}\right)$. Thus, by Propositions 4.5 and 4.8 , if we write $q_{t}\left(x_{0}\right)^{-1} \tilde{\kappa}_{Y_{t}}=\sum_{i, j} \tilde{g}_{i \bar{j}}(t, w) d w_{i} \wedge d \bar{w}_{j}$ on $B$, we get $\lambda I \leq\left(\tilde{g}_{i \bar{j}}\right) \leq \Lambda I$ and $\left|\tilde{g}_{i \bar{j}}\right|_{C^{k}(B)} \leq C_{k}$ where $\lambda, \Lambda, C_{k}>0$ are independent of $x_{0}$ and $t$. Putting $\bar{\kappa}_{t}:=q_{t}\left(x_{0}\right)^{-1} \tilde{\kappa}_{Y_{t}}$ and $\bar{\varphi}_{t}(s):=q_{t}\left(x_{0}\right)^{-1} \varphi_{t}(s)$, the Monge-Ampère equation (4.28) becomes as follows on $B$;

$$
\operatorname{det}\left(\tilde{g}_{i \bar{j}}+\frac{i}{2 \pi} \partial_{i \bar{j}} \bar{\varphi}_{t}(s)\right)^{2}=e^{s F_{t}+a_{t}(s)} \operatorname{det}\left(\tilde{g}_{i \bar{j}}\right), \quad \partial_{i \bar{j}} \bar{\varphi}_{t}(s)=\frac{\partial^{2} \bar{\varphi}_{t}(s)}{\partial w_{i} \bar{\partial} w_{j}}
$$


We find $\left|\partial_{i \bar{j}} \bar{\varphi}_{t}(s)\right|_{C^{0}(B)} \leq C$ and $\left|s F_{t}+a_{t}(s)\right|_{C^{2}(B)} \leq C$ by Theorem 4.2 and Proposition 4.7, which, together with Proposition 4.2, yields that there exists $\alpha>0$ independent of $t$ and $x$ such that $\left|\bar{\varphi}_{t}(s)\right|_{C^{2+\alpha}\left(B^{\prime}\right)} \leq C$ where $B^{\prime}=B\left(y_{0}, \epsilon / 2\right)$. Thus, $\tilde{g}_{i \bar{j}}+\frac{i}{2 \pi} \partial_{i \bar{j}} \bar{\varphi}_{t}(s)$ is uniformly elliptic and Hölder continuous on $B^{\prime}$. From the same argument as in [Si, Chap.II, (1.4)], it follows that arbitrary $C^{k}$-norm of $\bar{\varphi}_{t}(s)$ on $B^{\prime}$ is uniformly bounded and thus so is the covariant derivative of curvature of $q_{t}\left(x_{0}\right)^{-1} \kappa_{t}(s)$. Rewriting this in terms of the metric $\kappa_{t}(s)$, we get the assertion. When $q_{t}\left(x_{0}\right) \leq \delta^{-1}|t|$, as $|t|^{-1} \mu_{|t|}^{*} \tilde{\kappa}_{\mathcal{X}_{t}}=\omega_{\mathcal{X}_{1}}$ by the map $\mu_{|t|}(x)=|t| \cdot x$, we also get the assertion by the same argument as above because the injectivity radius of $\left(\mathcal{X}_{1}, \omega_{\mathcal{X}_{1}}\right)$ is positive.

\section{$\S 5$. Reduction to the ALE Instanton}

5.1 Estimates of Anomaly. Let us consider the situation in $\S 4.2$ and keep notations there. In this section, we assume that $\pi: \mathcal{Y} \rightarrow D$ is a degenerating family of 2-elementary $K 3$ surfaces with involution $\iota$ and $L$ is $\iota$-invariant. Thus, $\kappa_{Y_{t}}$ is also $\iota$-invariant by the uniqueness of Ricci-flat Kähler metric. As $o$ is a fixed point of $\iota$, according to the type in (2.11), we fix the local coordinates $(x, y, z, t)$ as in Proposition 2.3. Relative to this local coordinates, we can construct $\tilde{\kappa}_{Y_{t}}$ as in (4.18) and $\omega_{Y_{t}}$ as in (4.25). By taking the average of the action of $\iota$ if necessary, we may assume that both $\tilde{\kappa}_{Y_{t}}$ and $\omega_{Y_{t}}$ are $\iota$-invariant, and so is $\tilde{\kappa}_{t}(s)$.

Let $\widetilde{\mathrm{Td}}\left(Y_{t} ; \kappa_{Y_{t}}, \tilde{\kappa}_{Y_{t}}\right)$ be the Bott-Chern secondary class associated to the Todd genus and $\kappa_{Y_{t}}, \tilde{\kappa}_{Y_{t}}$. By definition ([B-C], [B-G-S, I (e)]), we get

$$
\begin{aligned}
\widetilde{\operatorname{Td}}\left(Y_{t} ; \kappa_{Y_{t}}, \tilde{\kappa}_{Y_{t}}\right)^{(2,2)}= & \frac{1}{24} \int_{1}^{0}\left(\operatorname{Tr} \kappa_{t}(s)^{-1} \dot{\kappa}_{t}(s)\right) c_{2}\left(R_{t}(s)\right) d s \\
& +\left.\frac{1}{24} \int_{1}^{0} c_{1}\left(R_{t}(s)\right) \cdot \frac{d}{d \epsilon}\right|_{\epsilon=0} c_{2}\left(R_{t}(s)+\epsilon \frac{\dot{\kappa}_{t}(s)}{\kappa_{t}(s)}\right) d s
\end{aligned}
$$

where $\dot{\kappa}_{t}(s)=\partial_{s} \kappa_{t}(s)$. As in $\S 4.2$, we denote by $C$ a constant independent of $s \in[0,1], t \in D$ and $x \in Y_{t}$, though its value may change in each estimate. For a norm $|\cdot|,|\cdot|_{\kappa}$ means that it uses the metric $\kappa$ in measuring.

Lemma 5.1. For any $t \in D \backslash\{0\}$, one has

$$
\left|\int_{Y_{t}} \widetilde{\operatorname{Td}}\left(Y_{t} ; \kappa_{Y_{t}}, \tilde{\kappa}_{X_{t}}\right)^{(2,2)}\right| \leq C \sup _{s \in[0,1]}\left|\kappa_{t}(s)^{-1} \dot{\kappa}_{t}(s)\right|_{\infty, \kappa_{X_{t}}} .
$$

Proof. In the proof, every norms and volumes are those relative to $\kappa_{t}(s)$. By (5.1),

$$
\left|\int_{Y_{t}} \widetilde{\operatorname{Td}}\left(Y_{t} ; \kappa_{Y_{t}}, \tilde{\kappa}_{Y_{t}}\right)^{(2,2)}\right| \leq \sup _{s \in[0,1]}\left|\kappa_{t}(s)^{-1} \dot{\kappa}_{t}(s)\right|_{\infty} \int_{0}^{1} d s \int_{Y_{t}}\left|R_{t}(s)\right|^{2} d v_{t}(s) .
$$

Let $\tau_{t}(s)$ the scalar curvature of $\left(Y_{t}, \kappa_{t}(s)\right)$. As is well known (cf. [G]),

$$
24=\chi\left(Y_{t}\right)=\int_{Y_{t}} \frac{1}{32 \pi^{2}}\left(\left|R_{t}(s)\right|^{2}-4\left|\operatorname{Ric}\left(\kappa_{t}(s)\right)\right|^{2}+\tau_{t}(s)^{2}\right) d v_{t}(s) .
$$


Let $\theta_{1}, \theta_{2}$ be a local unitary frame and write $\operatorname{Ric}\left(\kappa_{t}(s)\right)=i\left(\rho_{1} \theta_{1} \bar{\theta}_{1}+\rho_{2} \theta_{2} \bar{\theta}_{2}\right)\left(\rho_{1}, \rho_{2} \in\right.$ $\mathbb{R})$. Then, $\tau_{t}(s)=\rho_{1}+\rho_{2}$ and $\operatorname{Ric}\left(\kappa_{t}(s)\right)^{2}=2 \rho_{1} \rho_{2} d v_{t}(s)$. Thus,

$$
\int_{Y_{t}} \tau_{t}(s)^{2} d v_{t}(s)=\int_{Y_{t}}\left|\operatorname{Ric}\left(\kappa_{t}(s)\right)\right|^{2} d v_{t}(s)+\int_{X_{t}} c_{1}\left(Y_{t}\right)^{2}=\int_{Y_{t}}\left|\operatorname{Ric}\left(\kappa_{t}(s)\right)\right|^{2} d v_{t}(s)
$$

because $c_{1}\left(Y_{t}\right)$ is cohomologous to zero which, together with (5.3), yields

$$
\int_{Y_{t}}\left|R_{t}(s)\right|^{2} d v_{t}(s)=32 \pi^{2}\left(24+3 \int_{Y_{t}}\left|\operatorname{Ric}\left(\kappa_{t}(s)\right)\right|^{2} d v_{t}(s)\right)
$$

The assertion follows from (5.2) and (5.5) together with Theorem 4.2.

Lemma 5.2. For any $t \in D \backslash\{0\}$, one has

$$
\left|\int_{Y_{t}} \widetilde{\operatorname{Td}}\left(Y_{t} ; \kappa_{Y_{t}}, \tilde{\kappa}_{Y_{t}}\right)\right| \leq C
$$

Proof. Let $\Delta_{t}(s)$ be the Laplacian of $\left(Y_{t}, \kappa_{t}(s)\right)$. Differentiating (4.28) by $s$, we get

$$
\Delta_{t}(s) \dot{\varphi}_{t}(s)=F_{t}+\dot{a}_{t}(s), \quad \int_{Y_{t}} \dot{\varphi}_{t}(s) \kappa_{t}(0)^{2}=0 .
$$

Multiplying $\left|\dot{\varphi}_{t}(s)\right|^{p-2} \dot{\varphi}_{t}(s)$ to the both hand sides, the integration by parts yields

$$
\left.\left.\int_{X_{t}}|d| \dot{\varphi}_{t}(s)\right|^{\frac{p}{2}}\right|_{\kappa_{t}(s)} ^{2} d v_{t}(s) \leq \frac{p^{2}}{4(p-1)} \int_{X_{t}}\left|s F_{t}+a_{t}(s)\right|\left|\dot{\varphi}_{t}(s)\right|^{p-1} d v_{t}(s)
$$

for any $p \geq 2$. By Theorem 4.2, (5.7) is also valid after changing the metric from $\kappa_{t}(s)$ to $\kappa_{t}(0)$. Applying Moser's iteration argument ([Ko, pp.298-299]), we get

$$
\left|\dot{\varphi}_{t}(s)\right|_{\infty} \leq C\left\|\dot{\varphi}_{t}(s)\right\|_{L^{2}, \kappa_{t}(s)}
$$

where $C$ depends only on the constants in Theorem 4.2, the Sobolev constant of $\left(Y_{t}, \kappa_{t}(0)\right)$ and $\left|s F_{t}+a_{t}(s)\right|_{\infty}$. By Theorems 4.1 and $4.2, C$ is uniformly bounded because so is the Sobolev constant of $\left(Y_{t}, \kappa_{t}(0)\right)$. As in the proof of Theorem 4.1, we find that $\lambda_{t}$, the first eigenvalue of $\Delta_{t}(0)$, is also uniformly bounded away from zero; $\lambda_{t} \geq \lambda>0$ which, together with (5.7), $p=2$ and Theorem 4.2 , yields

$$
\left\|\dot{\varphi}_{t}(s)\right\|_{L^{2}}^{2} \leq \lambda^{-1}\left\|d \dot{\varphi}_{t}(s)\right\|_{L^{2}}^{2} \leq C \lambda^{-1}\left\|F_{t}+\dot{a}_{t}(s)\right\|_{L^{2}}\left\|\dot{\varphi}_{t}(s)\right\|_{L^{2}} \leq C|t|\left\|\dot{\varphi}_{t}(s)\right\|_{L^{2}}
$$

where norms are those relative to $\kappa_{t}(0)$. Comparing (5.8) and (5.9), we get

$$
\left|\dot{\varphi}_{t}(s)\right|_{\infty} \leq C|t|
$$

Take $x \in Y_{t} \cap W$, and let $(B, w)$ be the coordinate neighborhood centered at $x$ as in the proof of Theorem 4.3. Put $\hat{\kappa}_{t}(s):=q_{t}(x)^{-1} \kappa_{t}(s)$ for the rescaled metric on $B$. Let $\hat{\Delta}_{t}(s)=q_{t}(x) \Delta_{t}(s)$ be the Laplacian of $\left(X_{t}, \hat{\kappa}_{t}(s)\right)$. Then, (5.6) becomes

$$
\hat{\Delta}_{t}(s) \dot{\varphi}_{t}(s)=q_{t}(x)\left(F_{t}+\dot{a}_{t}(s)\right) \text {. }
$$


By Proposition 4.7, Theorem 4.2 and (5.10), the Schauder estimate ([G-T, Theorem 6.2]) applied to (5.11) yields (one can apply it because the geometry of $\left(B\left(0, r_{0}\right), \hat{\kappa}_{t}(s)\right)$ is bounded as shown in the proof of Theorem 4.3.)

$$
\begin{aligned}
\left|\dot{\varphi}_{t}(s)\right|_{C^{2+\alpha}\left(B\left(0, r_{0}\right)\right)} & \leq C\left(\left|\dot{\varphi}_{t}(s)\right|_{\infty}+\left|q_{t}(x)\left(F_{t}+\dot{a}_{t}(s)\right)\right|_{C^{\alpha}\left(B\left(0,2 r_{0}\right)\right)}\right) \\
& \leq C\left(|t|+q_{t}(x)\right) \leq C q_{t}(x) .
\end{aligned}
$$

As $\kappa_{t}(s)$ converges smoothly in $t$ to a Kähler metric on $Y_{0}$ outside of $W$ and thus $\left|\dot{\varphi}_{t}(s)\right|_{C^{2}\left(Y_{t} \backslash W\right)} \leq C$, it follows from (5.12) that, for any $x \in Y_{t} \cap W$,

$$
\left|\partial_{Y_{t}} \bar{\partial}_{Y_{t}} \dot{\varphi}_{t}(s)(x)\right|_{\hat{\kappa}_{t}(s)} \leq C q_{t}(x) \text {. }
$$

Rewriting (5.13) in terms of $\kappa_{t}(s)$, we get the assertion by Lemma 5.1 because

$$
\left|\kappa_{t}(s)^{-1} \dot{\kappa}_{t}(s)(x)\right|_{\kappa_{t}(s)}=\left|\partial_{Y_{t}} \bar{\partial}_{Y_{t}} \dot{\varphi}_{t}(s)(x)\right|_{\kappa_{t}(s)} \leq C q_{t}(x)^{-1} q_{t}(x) \leq C .
$$

Lemma 5.3. For any $t \in D \backslash\{0\}$, one has

$$
\left|\int_{Y_{t}} \widetilde{\operatorname{Td}}\left(Y_{t} ; \tilde{\kappa}_{Y_{t}}, \omega_{Y_{t}}\right)\right| \leq C
$$

Proof. Since (5.2) and (5.5) are also valid for $\tilde{\kappa}_{t}(s)$, we get

$$
\left|\int_{Y_{t}} \widetilde{\operatorname{Td}}\left(Y_{t} ; \tilde{\kappa}_{Y_{t}}, \omega_{Y_{t}}\right)\right| \leq C \sup _{s \in[0,1]}\left|\tilde{\kappa}_{t}(s)^{-1} \dot{\kappa}_{t}(s)\right|_{\infty}
$$

As $\partial_{s} \tilde{\kappa}_{t}(s)=\tilde{\kappa}_{Y_{t}}-\omega_{Y_{t}}$ by $(4.26)$, we get $\sup _{s \in[0,1]}\left|\tilde{\kappa}_{t}(s)^{-1} \dot{\tilde{\kappa}}_{t}(s)\right|_{\infty} \leq C$ by Theorem 4.1 which, together with (5.15), implies the assertion.

Proposition 5.1. For any $t \in D \backslash\{0\}$, one has

$$
\left|\int_{Y_{t}} \widetilde{\operatorname{Td}}\left(Y_{t} ; \kappa_{Y_{t}}, \omega_{Y_{t}}\right)\right| \leq C .
$$

Proof. Clear by Lemmas 5.2 and 5.3 because the Bott-Chern secondary class does not depend on a path joining two metrics ([B-C], [B-G-S I (e)]).

Let $\pi: Z \rightarrow D$ be the family of fixed curves of $\iota ; Z=\{x \in \mathcal{Y} ; \iota(x)=x\}$. Let $\kappa_{Z_{t}}(s)$ be the restriction of $\kappa_{t}(s)$ to $Z_{t} ; \kappa_{Z_{t}}(s)=\left.\kappa_{t}(s)\right|_{Z_{t}}$. By definition, $(5.16)$

$$
\begin{aligned}
& \int_{Z_{t}} c_{1}\left(\widetilde{\left.Y_{t}\right) c_{1}}\left(Z_{t}\right)\left(\kappa_{Y_{t}}, \tilde{\kappa}_{Y_{t}}\right)\right. \\
& =\int_{1}^{0} d s \int_{Z_{t}}\left(\operatorname{Tr} \kappa_{t}(s)^{-1} \dot{\kappa}_{t}(s)\right) c_{1}\left(Z_{t}, \kappa_{Z_{t}}(s)\right)+\kappa_{Z_{t}}(s)^{-1} \dot{\kappa}_{Z_{t}}(s) c_{1}\left(Y_{t}, \kappa_{t}(s)\right) .
\end{aligned}
$$


Lemma 5.4. For any $t \in D \backslash\{0\}$, one has

$$
\left|\int_{Z_{t}} c_{1} \widehat{\left(\widehat{\left.Y_{t}\right) c_{1}}\left(Z_{t}\right)\right.}\left(\kappa_{Y_{t}}, \tilde{\kappa}_{Y_{t}}\right)\right| \leq C .
$$

Proof. When $o=\operatorname{Sing} \mathcal{Y} \notin Z$, the assertion is obvious. Thus, we assume that $o \in Z_{0}$. By Proposition 2.5, $Z_{t}$ is a degenerating family of curves such that $Z_{0}$ has only one node at $o$. By (5.16), it is enough to show the followings;
(1) $\left|\operatorname{Tr} \kappa_{t}(s)^{-1} \dot{\kappa}_{t}(s)\right|_{\infty} \leq C|t|$
(2) $\left|c_{1}\left(Z_{t}, \kappa_{Z_{t}}(s)\right)\right|_{\infty, \kappa_{Z_{t}}} \leq C|t|^{-1}$,
(3) $\left|\kappa_{Z_{t}}(s)^{-1} \dot{\kappa}_{Z_{t}}(s)\right|_{\infty} \leq C$,
(4) $\left|c_{1}\left(X_{t}, \kappa_{t}(s)\right)\right|_{\infty, \kappa_{t}(s)} \leq C$.

As $\operatorname{Tr} \kappa_{t}(s)^{-1} \dot{\kappa}_{t}(s)=F_{t}+\dot{a}_{t}(s)=O(|t|)$ by Theorem 4.2, we get (1). (4) is proved in Theorem 4.2. (3) follows from (5.14) because, for any $v \in T Z_{t}$,

$$
-\left|\kappa_{t}(s)^{-1} \dot{\kappa}_{t}(s)\right|_{\infty} \leq \kappa_{t}(s)(v, v)^{-1} \partial_{s} \kappa_{t}(s)(v, v) \leq\left|\kappa_{t}(s)^{-1} \dot{\kappa}_{t}(s)\right|_{\infty} .
$$

As $\kappa_{t}(s)$ is $\iota$-invariant, $R_{t}(s)$ restricted to $Z_{t}$ splits as follows ([B-G-V, $\left.\left.\S 6.3(6.1)\right]\right)$;

$$
\left.R_{t}(s)\right|_{Z_{t}}=R\left(Z_{t}, \omega_{t}(s)\right) \oplus R\left(N_{Z_{t} / Y_{t}}\right)
$$

where $R\left(N_{Z_{t} / Y_{t}}\right)$ is the curvature of the normal bundle of $Z_{t}$ relative to the induced metric, and the splitting is orthogonal. Therefore, by Theorem 4.3, we get

$$
\left|c_{1}\left(Z_{t}, \kappa_{Z_{t}}(s)\right)\right|_{\infty}=\left|R\left(Z_{t}, \kappa_{Z_{t}}(s)\right)\right|_{\infty} \leq\left.\left|R_{t}(s)\right|_{Z_{t}}\right|_{\infty} \leq\left|R_{t}(s)\right|_{\kappa_{t}(s)} \leq C|t|^{-1} .
$$

Lemma 5.5. For any $t \in D \backslash\{0\}$, one has

$$
\mid \int_{Z_{t}} c_{1}\left(\widehat{\left.Y_{t}\right) c_{1}}\left(Z_{t}\right)\left(\tilde{\kappa}_{Y_{t}}, \omega_{Y_{t}}\right) \mid \leq C .\right.
$$

Proof. In the same way as the proof of Lemma 5.4, we may assume that $o \in Z_{0}$. Put $\tilde{\kappa}_{Z_{t}}(s):=\left.\tilde{\kappa}_{t}(s)\right|_{Z_{t}}$. By (5.16), it is enough to prove the following; for any $x \in Y_{t} \cap W$,

$$
\begin{aligned}
& \text { (1) }\left|\operatorname{Tr} \tilde{\kappa}_{t}(s)^{-1} \dot{\tilde{\kappa}}_{t}(s)\right|(x) \leq C q_{t}(x), \quad(2) \quad\left|c_{1}\left(Z_{t}, \tilde{\kappa}_{Z_{t}}(s)\right)\right|(x) \leq C q_{t}(x)^{-1} \text {, } \\
& \text { (3) }\left|\tilde{\kappa}_{Z_{t}}(s)^{-1} \dot{\tilde{\kappa}}_{Z_{t}}(s)\right|(x) \leq C, \quad \text { (4) }\left|c_{1}\left(Y_{t}, \tilde{\kappa}_{t}(s)\right)\right|(x) \leq C
\end{aligned}
$$

where length is measured relative to $\tilde{\kappa}_{Z_{t}}$ and $\tilde{\kappa}_{Y_{t}}$. As $\tilde{\kappa}_{t}(s)^{-1} \dot{\tilde{\kappa}}_{t}(s)=\tilde{\kappa}_{Y_{t}}-\omega_{Y_{t}}$, we get (3) by Theorem 4.1 and (1) by Lemma 4.4. (4) follows from Proposition 4.7. Since $\tilde{\kappa}_{t}(s)$ is $\iota$-invariant, by the same argument as the proof of (2) in Lemma 5.2, we get (2) by Proposition 4.8.

Proposition 5.2. For any $t \in D \backslash\{0\}$, one has

$$
\mid \int_{Z_{t}} c_{1}\left(\widetilde{\left.Y_{t}\right) c_{1}}\left(Z_{t}\right)\left(\kappa_{Y_{t}}, \omega_{Y_{t}}\right) \mid \leq C .\right.
$$

Proof. Clear by Lemmas 5.4 and 5.5

Define $\tilde{\tau}_{M}\left(Y_{t}, \iota_{t}, \omega_{Y_{t}}\right)$ in the same manner as Definition 3.3. By Propositions 5.1 and 5.2, we get the following. 
Theorem 5.1. For any $t \in D \backslash\{0\}$, one has

$$
\left|\log \tau_{M}\left(Y_{t}, \iota_{t}\right)-\log \tilde{\tau}_{M}\left(Y_{t}, \iota_{t}, \omega_{Y_{t}}\right)\right| \leq C .
$$

5.2 Singularity of Type $(0,3)$ and Asymptotics of $\boldsymbol{\tau}_{\boldsymbol{M}}$. In this subsection, we assume that $\left(Y_{0}, \iota, o\right)$ is a singularity of type $(0,3)$ in the sense of $(2.11)$. Put $\tau_{M}(t):=\tau_{M}\left(Y_{t} / \iota_{t}, \omega_{Y_{t}}\right)$.

Theorem 5.2. As $t \rightarrow 0$, one has the following asymptotic formula:

$$
\log \tau_{M}(t)=-\frac{1}{8} \log |t|^{2}+o(\log |t|)
$$

Proof. Let $\Delta_{t}$ be the Laplacian of $\left(Y_{t}, \omega_{Y_{t}}\right)$. As $\omega_{Y_{t}}$ is $\iota$-invariant, $\Delta_{t}$ splits into $\Delta_{t}^{ \pm}$as in Lemma 3.1. Let $K_{t}^{ \pm}(s, x, y)$ be the heat kernel of $\Delta_{t}^{ \pm}$and $K_{t}(s, x, y)$ that of $\Delta_{t}$. Since $K_{t}^{ \pm}(s, x, y)=K_{t}(s, x, y) / 2 \pm K_{t}(s, x, \iota y) / 2$ and thus

$$
\operatorname{Tr} e^{-s \Delta_{t}^{+}}-\operatorname{Tr} e^{-s \Delta_{t}^{-}}=\int_{X_{t}} K_{t}\left(s, x, \iota_{t} x\right) d v_{X_{t}},
$$

it follows from [B-G-V, Theorem 6.11] that there exists $a_{i}(z, t) \in C^{\infty}\left(Z_{t}\right)$ such that

$$
\operatorname{Tr} e^{-s \Delta_{t}^{+}}-\operatorname{Tr} e^{-s \Delta_{t}^{-}} \sim \int_{Z_{t}}\left(\frac{a_{1}(z, t)}{s}+a_{0}(z, t)\right) d v_{Z_{t}}+O(s) \quad(s \rightarrow 0) .
$$

Put $a_{i}(t)=\int_{Z_{t}} a_{i}(z, t) d v_{Z_{t}}$. From (5.20-21) and Lemma 3.1, it follows that

$$
\begin{aligned}
\tau\left(Y_{t} / \iota_{t}, \omega_{Y_{t}}\right)= & \log \operatorname{det} \Delta_{t}^{-}-\log \operatorname{det} \Delta_{t}^{+} \\
= & \int_{0}^{1} \frac{d s}{s}\left(\operatorname{Tr} e^{-s \Delta_{t}^{+}}-\operatorname{Tr} e^{-s \Delta_{t}^{-}}-\frac{a_{-1}(t)}{s}-a_{0}(t)\right) \\
& +\int_{1}^{\infty} \frac{d s}{s}\left(\operatorname{Tr} e^{-s \Delta_{t}^{+}}-\operatorname{Tr} e^{-s \Delta_{t}^{-}}-1\right)+a_{-1}(t)-\Gamma^{\prime}(1) a_{0}(t)+\Gamma^{\prime}(1) \\
= & \int_{0}^{1} \frac{d s}{s}\left\{\int_{Y_{t}} K_{t}(s, x, \iota x) d v_{Y_{t}}-\int_{Z_{t}}\left(\frac{a_{1}(z, t)}{s}+a_{0}(z, t)\right) d v_{Z_{t}}\right\} \\
& +\int_{1}^{\infty} \frac{d s}{s}\left\{\int_{Y_{t}} K_{t}(s, x, \iota x) d v_{Y_{t}}-1\right\}+a_{-1}(t)-\Gamma^{\prime}(1) a_{0}(t)+\Gamma^{\prime}(1) .
\end{aligned}
$$

Let $\left(X, \omega_{X}\right)$ be the ALE instanton (see (6.1) below). By (4.25), we have $\left(Y_{t} \cap W, \omega_{Y_{t}}\right) \cong\left(X \cap B\left(|t|^{-1 / 2}\right),|t| \omega_{X}\right)$. Let $K(s, x, y)$ be the heat kernel of $\left(X, \omega_{X}\right)$. As $\omega_{Y_{t}}$ is Ricci-flat on $Y_{t} \cap W$, it follows from [C-L-Y, $\left.\S 3,4\right]$ that there exist constants $C, \gamma>0$ such that

$$
0<K_{t}(s, x, y) \leq C s^{-2} e^{-\frac{\gamma d(x, y)}{s}}, \quad\left|d_{x} K_{t}(s, x, y)\right| \leq C s^{-\frac{5}{2}} e^{-\frac{\gamma d(x, y)}{s}}
$$

for any $t \in D \backslash\{0\}, s \in(0,1]$ and $x, y \in Y_{t} \cap W$, and one has the same estimates for $K(s, x, y)$ for any $s>0$ and $x, y \in X$. By Duhamel's principle (cf. [B-B, pp.63-67]) together with (5.23), there exist $c, C>0$ such that

$$
\left|\int_{Y_{t} \cap W} K_{t}(s, x, \iota x) d v_{Y_{t}}-\int_{X \cap B\left(|t|^{-\frac{1}{2}}\right)} K\left(|t|^{-1} s, x, \iota x\right) d v_{X}\right| \leq C e^{-\frac{c}{s}}
$$


for any $t \in D \backslash\{0\}$ and $s \in(0,1]$. Put $J(T):=\int_{0}^{T} s^{-1} d s \int_{B(\sqrt{T})} K(s, x, \iota x) d v_{X}$. Since $W \cap Z_{t}=\emptyset$, there appears no contribution from $Z_{t}$ to the divergence of $\tau\left(Y_{t} / \iota_{t}, \omega_{Y_{t}}\right)$ which, together with (5.24), yields

$$
\int_{0}^{1} \frac{d s}{s} \int_{Y_{t} \cap W} K_{t}(s, x, \iota x) d v_{Y_{t}}=J\left(|t|^{-1}\right)+O\left(\int_{0}^{1} \frac{d s}{s} e^{-\frac{c}{s}}\right)=J\left(|t|^{-1}\right)+O(1) .
$$

As $\omega_{Y_{t}}$ is a smooth family of metrics outside of $Y_{t} \cap W$, one has

$$
\left|\int_{0}^{1} \frac{d s}{s}\left(\int_{Y_{t} \backslash W} K_{t}(s, x, \iota x) d v_{Y_{t}}-\int_{Z_{t}}\left(\frac{a_{1}(z, t)}{s}+a_{0}(z, t)\right) d v_{Z_{t}}\right)\right| \leq C .
$$

Let $\lambda_{k}(t)$ be the $k$-th eigenvalue of $\Delta_{t}$. Since the Sobolev constant of $\left(Y_{t}, \omega_{Y_{t}}\right)$ is uniformly bounded away from zero, it follows from [C-L] that there exists $N \geq 0$ such that, for any $t \in D$ and $k \geq N$, one has $\lambda_{k}(t) \geq C k^{1 / 2}$. By [Yo1, Theorem 5.1], $\lambda_{k}(t)$ is a continuous function on $D$ for any $k \geq 0$ and $\lambda_{1}(0)>0$. Thus, we get the bound $\lambda_{k}(t) \geq C k^{1 / 2}$ and $0 \leq \operatorname{Tr} e^{-s \Delta_{t}}-1 \leq C e^{-\lambda_{1}(0) s / 2}$ for $s \geq 1$, which yields

$$
\begin{aligned}
\int_{1}^{\infty} \frac{d s}{s}\left|\int_{Y_{t}} K(s, x, \iota x) d v_{Y_{t}}-1\right| & =\int_{1}^{\infty} \frac{d s}{s}\left|\operatorname{Tr} e^{-s \Delta_{t}^{+}}-\operatorname{Tr} e^{-s \Delta_{t}^{-}}-1\right| \\
& \leq \int_{1}^{\infty} \frac{d s}{s}\left(\operatorname{Tr} e^{-s \Delta_{t}}-1\right) \leq C
\end{aligned}
$$

Let $K\left(s, x, y ; \lambda \omega_{X}\right)$ be the heat kernel and $B\left(r ; \lambda \omega_{X}\right)$ the metric ball of radius $r$ of $\left(X, \lambda \omega_{X}\right)$. Let $d s_{\mathbb{C}^{2}}^{2}$ be the Euclidean metric of $\mathbb{C}^{2}$ and $K_{\mathbb{C}^{2} / \pm 1}(s, x, y)$ be the heat kernel of $\left(\mathbb{C}^{2} / \pm 1, d s_{\mathbb{C}^{2}}^{2}\right)$. Since $\left(X, \lambda \omega_{X}, \iota, o\right)$ converges to $\left(\mathbb{C}^{2} / \pm 1, d s_{\mathbb{C}^{2}}^{2}, \iota^{\prime}, 0\right)$ as $\lambda \rightarrow 0$ where $\iota^{\prime}\left(z_{1}, z_{2}\right)=\left(\sqrt{-1} z_{1}, \sqrt{-1} z_{2}\right)$, we get

$$
\begin{aligned}
& \lim _{T \rightarrow \infty} \frac{J(T)}{\log T}=\lim _{T \rightarrow \infty} \int_{0}^{1} d \sigma \int_{B\left(T^{\frac{1-\sigma}{2}} ; T^{-\sigma} \omega_{X}\right)} K\left(1, x, \iota x ; T^{-\sigma} \omega_{X}\right) d v_{T^{-\sigma} \omega_{X}} \\
& =\int_{0}^{1} d \sigma \int_{\mathbb{C}^{2} / \pm 1} K_{\mathbb{C}^{2} / \pm 1}\left(1, x, \iota^{\prime} x\right) d v_{\mathbb{C}^{2}}=\frac{1}{2} \int_{\mathbb{C}^{2}}\left(\frac{e^{-\frac{|z-i z|^{2}}{4 s}}}{(4 \pi s)^{2}}+\frac{e^{-\frac{|z+i z|^{2}}{4 s}}}{(4 \pi s)^{2}}\right) d v_{\mathbb{C}^{2}}=\frac{1}{4} .
\end{aligned}
$$

As $\log \tau_{M}(t)=J\left(|t|^{-1}\right)+O(1) \quad(t \rightarrow 0)$ by (5.22), (5.26-27) and (1.1), the assertion follows from (5.28) because $\pi: Z \rightarrow D$ is a smooth morphism and thus $\log \tau\left(Z_{t}, \omega_{Z_{t}}\right)$ is bounded on $D$. 


\section{$\S 6$. Singularity of $\tau_{M}$ along the Discriminant Locus}

6.1 Heat Kernel on the ALE Instanton. Consider the following affine quadric with involution and the fixed locus;

$$
\begin{aligned}
& X:=\left\{\left(z_{1}, z_{2}, z_{3}\right) \in \mathbb{C}^{3} ; z_{1} z_{2}-z_{3}^{2}=1\right\}, \quad \iota\left(z_{1}, z_{2}, z_{3}\right)=\left(z_{1}, z_{2},-z_{3}\right), \\
& X^{\iota}=Z:=\left\{\left(z_{1}, z_{2}\right) \in \mathbb{C}^{2} ; z_{1} z_{2}=1\right\} .
\end{aligned}
$$

Let $\omega_{X}=\partial_{X} \bar{\partial}_{X} q$ be the Kronheimer's ALE metric on $X([\mathrm{Kr}])$ as in $\S 4.2$, and put $\omega_{Z}:=\left.\omega_{X}\right|_{Z}$ for its restriction to $Z$. Fix $o \in X$ and put $r(x):=\operatorname{dist}(o, x)$. Let $B(\rho)$ be the metric ball of radius $\rho$ centered at $o$. By Kronheimer $[\mathrm{Kr}]$, there exist $c, C_{\alpha}>0$ with the following properties; (1) For any $y \in X$, the injectivity radius at $y$ is greater than $j_{y}=c(1+r(y))$. (2) On the metric ball $B\left(y, j_{y}\right)$ with the normal coordinates $x=\left(x_{1}, \cdots, x_{4}\right)$, the metric tensor $g(x)=\sum g_{i j}(x) d x_{i} d x_{j}$ satisfies

$$
\sup _{x \in B\left(y, j_{y}\right)}\left|\partial^{\alpha}\left(g_{i j}(x)-\delta_{i j}\right)\right| \leq C_{\alpha}(1+r(y))^{-|\alpha|} .
$$

For simplicity, we assume $c=2$ by considering $(2 c)^{-1} \omega_{X}$ if necessary. Let $K(t, x, y)$ be the heat kernel of $\left(X, \omega_{X}\right)$ and define its parametrix ([B-G-V, Theorem 2.26]);

$$
p(t, x, y):=(4 \pi t)^{-\frac{m}{2}} e^{-\frac{d(x, y)^{2}}{4 t}}\left\{u_{0}(x, y)+t u_{1}(x, y)\right\}
$$

where $u_{i}(\cdot, y) \in C^{\infty}\left(B\left(y, j_{y}\right)\right)$. (In [B-G-V], $\Phi_{i}$ is used instead of $u_{i}$.)

Lemma 6.1. For any $y \in X$ and $(x, t) \in B\left(y, j_{y} / 2\right) \times\left[0,1+|y|^{2}\right]$, one has

$$
|K(t, x, y)-p(t, x, y)| \leq C\left(c, C_{\alpha}\right)\left(1+r(y)^{2}\right)^{-2} e^{-\frac{\gamma d(x, y)^{2}}{t}} .
$$

Proof. Let $B(r)$ be the ball of radius $r<1$ in $\mathbb{R}^{4}$. Let $(M, g)$ be a Riemannian 4-manifold such that $B(1)$ is embedded into $M$ and $g=\sum g_{i j} d x_{i} d x_{j}$ on $B(1)$. Suppose that $\left|\partial^{k}\left(g_{i j}(x)-\delta_{i j}\right)\right| \leq A_{k}$ for any $k \geq 0$ and $x \in B(1)$. Let $L(t, x, y)$ be the heat kernel of $(M, g)$. By [C-L-Y, $\S 3,4]$, there exists $r, C, \gamma>0$ depending only on $A_{k}(k \leq 6)$ and $r$ such that, for any $(x, y) \in B(r) \times B(r)$ and $t \in(0,1]$,

$$
0<L(t, x, y) \leq C t^{-2} e^{-\frac{\gamma d(x, y)^{2}}{t}}, \quad\left|d_{x} L(t, x, y)\right| \leq C t^{-\frac{5}{2}} e^{-\frac{\gamma d(x, y)^{2}}{t}} .
$$

Let $L^{\prime}(t, x, y)$ be the Dirichlet heat kernel of $(B(1), g)$ which satisfies the same estimates (6.4). By Duhamel's principle, for any $(x, y) \in B(r) \times B(r)$ and $t \in(0,1]$,

$$
\left|L(t, x, y)-L^{\prime}(t, x, y)\right| \leq C e^{-\frac{c_{0}}{t}}
$$

where $c_{0}, C>0$ depend only on $C_{k}$. Since $C^{k}$-norm of $g_{i j}$ is bounded by $A_{k}(k=6$ is enough), it follows from the usual asymptotic expansion of the heat kernel that there exits a constant $C, \gamma>0$ depending only on $A_{k}(k \leq 6)$ and $r$ such that, for any $(x, y) \in B(r) \times B(r)$ and $t \in(0,1]$,

$$
\left|L^{\prime}(t, x, y)-p_{L^{\prime}}(t, x, y)\right| \leq C e^{-\frac{\gamma d(x, y)^{2}}{t}}
$$


where $p_{L^{\prime}}(t, x, y)$ is defined by the formula (6.3) for $L^{\prime}$ and $d(x, y)$ is the distance relative to the metric $g$. Comparing (6.5) and (6.6), we find

$$
\left|L(t, x, y)-p_{L}(t, x, y)\right| \leq C e^{-\frac{\gamma d(x, y)^{2}}{t}} .
$$

For any $y \in X$, consider the rescaled space $\left(X, j_{y}^{-2} \omega_{X}\right)$ and its heat kernel $L(t, x, y)$. As $j_{y}^{4} K\left(j_{y}^{2} t, x, y\right)=L(t, x, y)$ and $j_{y}^{4} p\left(j_{y}^{2} t, x, y\right)=p(t, x, y)$, it follows from (6.7) that

$$
\left|K\left(j_{y}^{2} t, x, y\right)-p\left(j_{y}^{2} t, x, y\right)\right| \leq C(1+r(y))^{-4} \exp \left(-\frac{\gamma d\left(x, y ; \omega_{X}\right)^{2}}{j_{y}^{2} t}\right), \quad(t \in(0,1]) .
$$

The assertion follows from (6.8) by putting $s=j_{y}^{2} t$.

Let $\mathcal{N}_{Z / X}$ be the normal bundle of $Z$ in $X$ and $v$ its fiber coordinate. Set $\Omega:=\left\{(z, v) \in \mathcal{N}_{Z / X} ;\|v\|_{z} \leq r(z)+1\right\}, \Omega(a):=\left\{(z, v) \in \Omega ; r\left(\exp _{z}(v)\right) \leq a\right\}$.

Via the exponential map, identify $\Omega$ with a tubular neighborhood of $Z$ in $X$. Let $d v_{Z}$ be the volume form of $\left(Z, \omega_{Z}\right)$. Then, the volume form of $\left(X, \omega_{X}\right)$ can be written on $\Omega$ as follows; $d v_{X}(x)=J(z, v) d v d \bar{v} d v_{Z}(z)$ where $x=\exp _{z}(v)$. Set

$$
q_{i}(z, v):=J(z, v) u_{i}(x, \iota(x)), \quad q_{i}^{(k)}(z, v):=\left.\frac{1}{k !} \partial_{t}^{k}\right|_{t=0} q_{i}(z, t v) .
$$

In the sequel, $C$ denotes a constant which depends only on $C_{\alpha}(|\alpha| \leq 6), c$ and $\gamma$. By definition, we get $q_{0}(z, 0)=1$. By $(6.2)$, the following is clear.

Lemma 6.2. For any $(z, v) \in \Omega$, one has the following estimates;

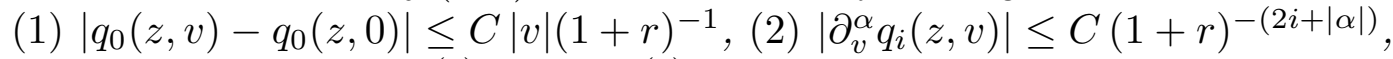

(3) $\left|q_{0}(z, v)-q_{0}(z)-q_{0}^{(1)}(z, v)-q_{0}^{(2)}(z, v)\right| \leq C|v|^{3}(1+r)^{-3}$,

(4) $\left|q_{1}(z, v)-q_{1}(z, 0)\right| \leq C|v|(1+r)^{-3}$.

In (2), $|\alpha|>0$ when $i=0$ and $|\alpha| \geq 0$ when $i=1$.

Let $d x d y=\frac{\sqrt{-1}}{2} d v d \bar{v}$ be the volume form on the fiber of $\mathcal{N}_{Z / X}$. Set $I_{1}(s, z):=\int_{|v| \leq r(z)+1} s^{-2} e^{-|v|^{2} / s}\left(q_{0}(z, v)-q_{0}(z)-q_{0}^{(1)}(z, v)-q_{0}^{(2)}(z, v)\right) d x d y$.

Lemma 6.3. For any $T>1$, one has

$$
\left|\int_{0}^{T} \frac{d s}{s} \int_{r \leq \sqrt{T}} I_{1}(s, z) d v_{Z}\right| \leq C(\log T+1) .
$$

Proof. Suppose $r \geq \sqrt{s}$. It follows from Lemma 6.2 (3) that

$\left|I_{1}(s, z)\right| \leq \int_{|v| \leq r+1} C e^{-\frac{|v|^{2}}{s}} \frac{|v|^{3}}{s^{2}} \frac{d x d y}{(1+r)^{3}} \leq C \int_{\rho \leq r+1} e^{-\frac{\rho^{2}}{s}} \frac{\rho^{4} d \rho}{s^{2}(1+r)^{3}} \leq C \frac{s^{\frac{1}{2}}}{(1+r)^{3}}$

which yields

$$
\left|\int_{1}^{T} \frac{d s}{s} \int_{\sqrt{s} \leq r \leq \sqrt{T}} I_{1}(z, s) d v_{Z}\right| \leq C \int_{1}^{T} \frac{d s}{s} \int_{\sqrt{s}}^{\infty} s^{\frac{1}{2}}(1+r)^{-3} r d r \leq C \log T .
$$


Suppose $r \leq \sqrt{s}$. It follows from Lemma 6.2 (1) that

$\left|\int_{|v| \leq r+1} e^{-\frac{|v|^{2}}{s}}\left(q_{0}(z, v)-q_{0}(z)\right) \frac{d x d y}{s^{2}}\right| \leq C \int_{|v| \leq r+1} e^{-\frac{|v|^{2}}{s}} \frac{|v| d x d y}{s^{2}(1+r)} \leq C \frac{(1+r)^{2}}{s^{2}}$.

Similarly, using Lemma 6.2 (2), we get

$$
\left|\int_{|v| \leq r+1} e^{-\frac{|v|^{2}}{s}} q_{0}^{(2)}(z, v) \frac{d x d y}{s^{2}}\right| \leq C \int_{|v| \leq r+1} e^{-\frac{|v|^{2}}{s}} \frac{|v|^{2} d x d y}{s^{2}(1+r)^{2}} \leq C \frac{(1+r)^{2}}{s^{2}} .
$$

Since $\int_{|v| \leq r+1} e^{-\frac{|v|^{2}}{s}} v^{k} d x d y=0(k>0)$, we get

$$
\int_{|v| \leq r+1} s^{-2} e^{-\frac{|v|^{2}}{s}} q_{0}^{(1)}(z, v) d x d y=0
$$

which, together with (6.12-13), yields

$$
\left|\int_{1}^{T} \frac{d s}{s} \int_{r \leq \sqrt{s}} I_{1}(z, s) d v_{Z}\right| \leq C \int_{1}^{T} \frac{d s}{s} s^{-2} \int_{r \leq \sqrt{s}}(1+r)^{2} r d r \leq C \log T .
$$

When $s \leq 1,1+r(z) \geq s$ for any $z \in Z$. Thus, by (6.10), we get

$$
\left|\int_{0}^{1} \frac{d s}{s} \int_{r \leq \sqrt{T}} I_{1}(z, s) d v_{Z}\right| \leq C \int_{0}^{1} \frac{d s}{s} \sqrt{s} \int_{r \leq \sqrt{T}}(1+r)^{-3} r d r \leq C .
$$

The assertion follows from (6.11) and (6.15-16).

Set $I_{2}(s, z):=\int_{|v| \leq r(z)+1} s^{-1} e^{-|v|^{2} / s}\left(q_{1}(z, v)-q_{1}(z, 0)\right) d x d y$.

Lemma 6.4. For any $T>1$, one has

$$
\left|\int_{0}^{T} \frac{d s}{s} \int_{r \leq \sqrt{T}} I_{2}(s, z) d v_{Z}\right| \leq C(\log T+1) .
$$

Proof. Suppose $r \geq \sqrt{s}$. It follows from Lemma 6.2 (4) that

$$
\left|I_{2}(s, z)\right| \leq C \int_{|v| \leq r+1} e^{-\frac{|v|^{2}}{s}} \frac{|v|}{s} \frac{d x d y}{(1+r)^{3}} \leq C \int_{\rho \leq r+1} e^{-\frac{\rho^{2}}{s}} \frac{\rho^{2}}{s} \frac{d \rho}{(1+r)^{3}} \leq C \frac{\sqrt{s}}{(1+r)^{3}}
$$

which yields

$$
\left|\int_{1}^{T} \frac{d s}{s} \int_{\sqrt{s} \leq r \leq \sqrt{T}} I_{2}(s, z) d v_{Z}\right| \leq C \int_{1}^{T} \frac{d s}{s} s^{\frac{1}{2}} \int_{\sqrt{s}}^{\infty}(1+r)^{-3} r d r \leq C \log T .
$$

Suppose $r \leq \sqrt{s}$. It follows from the same lemma that

$$
\left|I_{2}(s, z)\right| \leq C(1+r)^{-3} \int_{\rho \leq r+1} s^{-1} e^{-\frac{\rho^{2}}{s}} \rho^{2} d \rho \leq C s^{-\frac{1}{2}}(1+r)^{-1}
$$


which yields

$$
\left|\int_{1}^{T} \frac{d s}{s} \int_{r \leq \sqrt{s}} I_{2}(s, z) d v_{Z}\right| \leq C \int_{1}^{T} \frac{d s}{s} s^{-\frac{1}{2}} \int_{0}^{\sqrt{s}}(1+r)^{-1} r d r \leq C \log T
$$

When $s \leq 1$, it follows from (6.17) that

$$
\left|\int_{0}^{1} \frac{d s}{s} \int_{r \leq \sqrt{T}} I_{2}(s, z) d v_{Z}\right| \leq C \int_{0}^{1} \frac{d s}{s} s^{\frac{1}{2}} \int_{0}^{\infty}(1+r)^{-3} r d r \leq C .
$$

The assertion follows from (6.17), (6.19) and (6.21).

Lemma 6.5. For any $T>1$, one has

$$
\left|\int_{0}^{T} \frac{d s}{s} \int_{\Omega(\sqrt{T})}\{K(s, x, \iota x)-p(s, x, \iota x)\} d v_{X}\right| \leq C(\log T+1) .
$$

Proof. Put $E(s, x):=K(s, x, \iota x)-p(s, x, \iota x), I_{3}(s)=\int_{\Omega(\sqrt{T}) \backslash \Omega(\sqrt{s})} E(s, x) d v_{X}$, and $I_{4}(s)=\int_{\Omega(\sqrt{s})} E(s, x) d v_{X}$. Suppose $1 \leq s \leq T$. By Lemma 6.1, we get

$$
\begin{aligned}
\left|I_{3}(s)\right| & \leq C \int_{\Omega(\sqrt{T}) \backslash \Omega(\sqrt{s})}\left(1+r^{2}\right)^{-2} e^{-\frac{\gamma|v|^{2}}{s}} d v_{Z} d x d y \\
& \leq C \int_{\sqrt{s}}^{\sqrt{T}} \frac{r d r}{\left(1+r^{2}\right)^{2}} \int_{|v| \leq r} e^{-\frac{\gamma|v|^{2}}{s}} d x d y \leq C \int_{\sqrt{s}}^{\sqrt{T}} \frac{d r}{r^{3}} \leq C .
\end{aligned}
$$

As $\left(X, \omega_{X}\right)$ is Ricci-flat, we get a bound ([L-Y, Theorem 3.2]); $K(t, x, y) \leq C t^{-2}$ for any $t>0$ and $x, y \in X$. Then, we have

$$
\begin{aligned}
\left|I_{4}(s)\right| & \leq C \int_{r \leq \sqrt{s}} d v_{Z} \int_{|v| \leq r}\left(\frac{1}{s^{2}}+\frac{1}{s\left(1+r^{2}\right)}\right) d x d y \\
& \leq C \frac{1}{s^{2}} \int_{r \leq \sqrt{s}} r^{2} d v_{Z}+C \int_{r \leq \sqrt{s}} d v_{Z} \leq C \frac{1}{s^{2}} \int_{r \leq \sqrt{s}} r^{3} d r+C \frac{1}{s} \int_{r \leq \sqrt{s}} r d r \leq C .
\end{aligned}
$$

Suppose that $s \leq 1$. By Lemma 6.1, we get

$\left|I_{3}(s)+I_{4}(s)\right| \leq C \int_{\Omega(\sqrt{T})} \frac{e^{-\frac{\gamma|v|^{2}}{s}} d v_{X}}{\left(1+r^{2}\right)^{2}} \leq C s \int_{0}^{\sqrt{T}} \frac{r d r}{\left(1+r^{2}\right)^{2}} \int_{\mathbb{C}} e^{-\frac{\gamma|v|^{2}}{s}} \frac{d x d y}{s} \leq C s$.

Together with (6.23-25), we get

$$
\left|\int_{0}^{T} \frac{d s}{s}\left(I_{3}(s)+I_{4}(s)\right)\right| \leq C \int_{1}^{T} \frac{d s}{s}+C \int_{0}^{1} \frac{d s}{s} s \leq C(\log T+1)
$$


Lemma 6.6. Put $q_{0 ; 1 \overline{1}}(z, 0)=\left.\partial_{v} \partial_{\bar{v}}\right|_{v=0} q_{0}(z, v)$. For any $T>1$, one has

$$
\begin{aligned}
& \int_{B(\sqrt{T})} K(s, x, \iota x) d v_{X}-\frac{1}{16 \pi s} \int_{r \leq \sqrt{T}} d v_{Z}-\frac{1}{16 \pi} \int_{r \leq \sqrt{T}} q_{0 ; 1 \overline{1}}(z, 0) d v_{Z} \\
& =\int_{B(\sqrt{T}) \backslash \Omega(\sqrt{T})} K(s, x, \iota x) d v_{X}+I_{3}(s)+I_{4}(s)-\frac{1}{16 \pi s} \int_{r \leq \sqrt{T}} e^{-\frac{r^{2}}{s}} d v_{Z} \\
& \quad-\frac{1}{16 \pi} \int_{r \leq \sqrt{T}} q_{0 ; 1 \overline{1}}(z, 0) e^{-\frac{r^{2}}{s}}\left(1+\frac{r^{2}}{s}\right) d v_{Z}+\int_{r \leq \sqrt{T}}\left(I_{1}(s, z)+I_{2}(s, z)\right) d v_{Z} .
\end{aligned}
$$

Proof. By the definition of $p(s, x, y), J(z, v)$ and $q_{i}^{(k)}(z, v)$, we get

$$
\begin{aligned}
& (4 \pi s)^{2} p(s, x, \iota x) J(z, v)=e^{-\frac{|v|^{2}}{s}}\left\{q_{0}(z, 0)+q_{0}^{(1)}(z, v)+q_{0}^{(2)}(z, v)\right\}+s e^{-\frac{|v|^{2}}{s}} q_{1}(z, 0) \\
& +e^{-\frac{|v|^{2}}{s}}\left\{q_{0}(z, v)-q_{0}(z, 0)-q_{0}^{(1)}(z, v)-q_{0}^{(2)}(z, v)\right\}+s e^{-\frac{|v|^{2}}{s}}\left\{q_{1}(z, v)-q_{1}(z, 0)\right\}
\end{aligned}
$$

which, together with $q_{0}(z, 0)=1, q_{1}(z, 0)=0\left(\operatorname{Ric}\left(\omega_{X}\right)=0\right),(6.14)$ and the definition of $I_{1}$ and $I_{2}$, yields

$$
\begin{aligned}
& \int_{\Omega(\sqrt{T})} p(s, x, \iota x) d v_{X}-\int_{r \leq \sqrt{T}}\left(I_{1}(s, z)+I_{2}(s, z)\right) d v_{Z} \\
& =\int_{\Omega(\sqrt{T})}\left\{(4 \pi s)^{-2} e^{-\frac{|v|^{2}}{s}}+(4 \pi s)^{-2} e^{-\frac{|v|^{2}}{s}} q_{0 ; 1 \overline{1}}(z, 0)|v|^{2}\right\} d v_{X} \\
& =\int_{r \leq \sqrt{T}} d v_{Z} \int_{|v| \leq r+1} e^{-\frac{r^{2}}{s}} \frac{d x d y}{(4 \pi s)^{2}}+\int_{r \leq \sqrt{T}} q_{0 ; 1 \overline{1}}(z, 0) d v_{Z} \int_{|v| \leq r+1} e^{-\frac{r^{2}}{s}} r^{2} \frac{d x d y}{(4 \pi s)^{2}} \\
& =\frac{1}{16 \pi s} \int_{r \leq \sqrt{T}} d v_{Z}-\frac{1}{16 \pi s} \int_{r \leq \sqrt{T}} e^{-\frac{(r+1)^{2}}{s}} d v_{Z} \\
& \quad+\frac{1}{16 \pi} \int_{r \leq \sqrt{T}} q_{0 ; 1 \overline{1}}(z, 0) d v_{Z}-\frac{1}{16 \pi} \int_{r \leq \sqrt{T}} q_{0 ; 1 \overline{1}}(z, 0) e^{-\frac{(r+1)^{2}}{s}}\left(1+\frac{(r+1)^{2}}{s}\right) d v_{Z} .
\end{aligned}
$$

Therefore, we get

$$
\begin{aligned}
& \int_{\Omega(\sqrt{T})} p(s, x, \iota x) d v_{X}-\frac{1}{16 \pi s} \int_{r \leq \sqrt{T}} d v_{Z}-\frac{1}{16 \pi} \int_{r \leq \sqrt{T}} q_{0 ; 1 \overline{1}}(z, 0) d v_{Z} \\
& =-\frac{1}{16 \pi s} \int_{r \leq \sqrt{T}} e^{-\frac{(r+1)^{2}}{s}} d v_{Z}-\frac{1}{16 \pi} \int_{r \leq \sqrt{T}} q_{0 ; 1 \overline{1}}(z, 0) e^{-\frac{(r+1)^{2}}{s}}\left(1+\frac{(r+1)^{2}}{s}\right) d v_{Z} \\
& \quad+\int_{r \leq \sqrt{T}}\left(I_{1}(s, z)+I_{2}(s, z)\right) d v_{Z}
\end{aligned}
$$

which, together with the definition of $I_{3}, I_{4}$ and the following, yields the assertion;

$$
\begin{aligned}
& \int_{B(\sqrt{T})} K(s, x, \iota x) d v_{X}-\frac{1}{16 \pi s} \int_{r \leq \sqrt{T}} d v_{Z}-\frac{1}{16 \pi} \int_{r \leq \sqrt{T}} q_{0 ; 1 \overline{1}}(z, 0) d v_{Z} \\
& =\int_{B(\sqrt{T}) \backslash \Omega(\sqrt{T})} K(s, x, \iota x) d v_{X}+\int_{\Omega(\sqrt{T})}\{K(s, x, \iota x)-p(s, x, \iota x)\} d v_{X} \\
& \quad+\int_{\Omega(\sqrt{T})} p(s, x, \iota x) d v_{X}-\frac{1}{16 \pi s} \int_{r \leq \sqrt{T}} d v_{Z}-\frac{1}{16 \pi} \int_{r \leq \sqrt{T}} q_{0 ; 1 \overline{1}}(z, 0) d v_{Z}
\end{aligned}
$$


Lemma 6.7. For any $s \in(0,1]$, one has

$$
\left|\int_{B(\sqrt{T})} K(s, x, \iota x) d v_{X}-\frac{1}{s} \int_{r \leq \sqrt{T}} \frac{d v_{Z}}{16 \pi}-\int_{r \leq \sqrt{T}} q_{0 ; 1 \overline{1}}(z, 0) \frac{d v_{Z}}{16 \pi}\right| \leq C \sqrt{s} .
$$

Proof. Put $I_{5}(s):=\int_{B(\sqrt{T}) \backslash \Omega(\sqrt{T})} K(s, x, \iota x) d v_{X}, I_{6}(s):=\int_{r \leq \sqrt{T}} s^{-1} e^{-\frac{r^{2}}{s}} d v_{Z}$, and $I_{7}(s):=\int_{r \leq \sqrt{T}} q_{0 ; 1 \overline{1}}(z, 0) e^{-\frac{r^{2}}{s}}\left(1+\frac{r^{2}}{s}\right) d v_{Z}$. For any $x \in B(\sqrt{T}) \backslash \Omega(\sqrt{T})$, we get $2 d(x, \iota x) \geq r(x)+1$ which, together with (6.23), yields that, for any $s>0$,

$$
\begin{aligned}
& \left|I_{5}(s)\right| \leq C \int_{X} s^{-2} e^{-\frac{r^{2}+1}{s}} d v_{X} \leq C e^{-\frac{1}{s}}, \quad\left|I_{6}(s)\right| \leq C \int_{Z} s^{-1} e^{-\frac{r^{2}+1}{s}} d v_{Z} \leq C e^{-\frac{1}{s}}, \\
& \left|I_{7}(s)\right| \leq C \int_{Z} e^{-\frac{(r+1)^{2}+1}{2 s}}\left(1+\frac{(1+r)^{2}}{s}\right) \frac{d v_{Z}}{(1+r)^{2}} \leq C \int_{0}^{\infty} \frac{e^{-\frac{(1+r)^{2}+1}{4 s}} r d r}{(1+r)^{2}} \leq C e^{-\frac{1}{8 s}} .
\end{aligned}
$$

By (6.10), (6.17) and (6.24), we get $\int_{r \leq \sqrt{T}}\left(\left|I_{1}(s, z)\right|+\left|I_{2}(s, z)\right|\right) d v_{Z} \leq C \sqrt{s}$ and $\left|I_{3}(s)+I_{4}(s)\right| \leq C s$ for any $s \in(0,1]$ which, together with (6.30) and Lemma 6.6, yields the assertion.

Proposition 6.1. For any $T>1$, one has

$$
\left|\int_{0}^{T} \frac{d s}{s}\left\{\int_{B(\sqrt{T})} K(s, x, \iota x) d v_{X}-\int_{r \leq \sqrt{T}}\left(\frac{d v_{Z}}{16 \pi s}+q_{0 ; 1 \overline{1}}(z, 0) \frac{d v_{Z}}{16 \pi}\right)\right\}\right| \leq C(\log T+1) .
$$

Proof. By Lemmas 6.3-7 together with (6.30), we get

$$
\text { L.H.S. } \leq C(\log T+1)+\int_{0}^{T} \frac{d s}{s}\left(\left|I_{5}(s)\right|+\left|I_{6}(s)\right|+\left|I_{7}(s)\right|\right) \leq C(\log T+1) .
$$

6.2 Singularity of Type $(2,1)$ and Asymptotics of $\tau_{\boldsymbol{M}}$. Let us consider the same situation as in $\S 5.2$. Here, we assume that $\left(Y_{0}, \iota, o\right)$ is of type $(2,1)$ in the sense of (2.11). By Proposition 2.3, $Z_{0}$ has only one node at $o$. In the sequel, we use the same notations as in $§ 5.2$.

Lemma 6.8. There exists a function $I(T)$ defined for $T>1$ such that, as $t \rightarrow 0$,

$$
\log \tau\left(Y_{t} / \iota_{t}, \omega_{Y_{t}}\right)=I\left(|t|^{-1}\right)+O(1), \quad|I(T)| \leq C(\log T+1) .
$$

Proof. Let $K_{t}(s, x, y)$ be the heat kernel of $\left(Y_{t}, \omega_{Y_{t}}\right)$ and

$$
K_{t}\left(s, x, \iota_{t} x\right) \sim\left(a_{1}(x, t) s^{-1}+a_{0}(x, t)+O(s)\right) \delta_{Z_{t}}(x) \quad(s \rightarrow 0)
$$

its pointwise asymptotic expansion ([B-G-V, Theorem 6.11]) where $\delta_{Z_{t}}$ is the Dirac $\delta$-function supported along $Z_{t}$ and $a_{i}(z, t)$ is a smooth function on $Z_{t}$. By Lemma 3.1 , we get in the same manner as $(5.25)$

$$
\begin{aligned}
\log \tau\left(Y_{t} / \iota_{t}, \omega_{Y_{t}}\right)= & \int_{0}^{1} \frac{d s}{s}\left\{\int_{Y_{t}} K_{t}(s, x, \iota x) d v_{Y_{t}}-\int_{Z_{t}}\left(\frac{a_{1}(z, t)}{s}+a_{0}(z, t)\right) d v_{Z_{t}}\right\} \\
& +\int_{1}^{\infty} \frac{d s}{s}\left\{\int_{Y_{t}} K_{t}(s, x, \iota x) d v_{Y_{t}}-1\right\}+a_{-1}(t)-\Gamma^{\prime}(1)\left(a_{0}(t)-1\right)
\end{aligned}
$$


where $a_{i}(t):=\int_{Y_{t}} a_{i}(x, t) d v_{Y_{t}}$. By the same argument as in the proof of Theorem 5.4 using (5.23) and Duhamel's principle, we get (5.24). Put

$I(T):=\int_{0}^{T} \frac{d s}{s}\left\{\int_{B(\sqrt{T})} K(s, x, \iota x) d v_{X}-\frac{1}{s} \int_{r \leq \sqrt{T}} \frac{d v_{Z}}{16 \pi}-\int_{r \leq \sqrt{T}} q_{0 ; 1 \overline{1}}(z, 0) \frac{d v_{Z}}{16 \pi}\right\}$.

By Lemma 6.7 and (5.24), the integrand of $d s / s$ in $I(T)$ should coincides with the asymptotic expansion (6.33) on $W \cap Y_{t}$ and we get $\int_{Y_{t} \cap W} a_{0}(z, t) d v_{Z_{t}}=\int_{r \leq|t|^{-\frac{1}{2}}} d v_{Z} / 16 \pi$ and $\int_{Y_{t} \cap W} a_{1}(z, t) d v_{Z_{t}}=\int_{r \leq|t|^{-\frac{1}{2}}} q_{0 ; 1 \overline{1}}(z, 0) d v_{Z} / 16 \pi$ which, together with $(6.33)$, (5.24) and the definition of $I(T)$, yields

$\int_{0}^{1} \frac{d s}{s} \int_{Y_{t} \cap W}\left\{K_{t}(s, x, \iota x)-\frac{a_{1}(x, t)}{s}-a_{0}(x, t)\right\} d v_{Y_{t}}=I\left(|t|^{-1}\right)+O\left(\int_{0}^{1} \frac{d s}{s} e^{-\frac{c}{s}}\right)$.

In the same way as $§ 5.2$, we get (5.27) which, together with (6.33-34) and Proposition 6.1, yields the assertion.

Theorem 6.1. As $t \rightarrow 0$, one has the following asymptotic formula:

$$
\log \tau_{M}\left(Y_{t}, \iota_{t}\right)=-\frac{1}{8} \log |t|+O(1)
$$

Proof. Let $\omega_{Z_{t}}:=\left.\omega_{Y_{t}}\right|_{Z_{t}}$ be the induced metric on $Z_{t}$. In the similar way as [B-B, Théorème 6.2], there exists an universal constant $\beta \in \mathbb{R}$ such that one has $\log \tau\left(Z_{t}, \omega_{Z_{t}}\right)=\beta \log |t|+O\left(\log \log |t|^{-1}\right)$ as $t \rightarrow 0$, which, together with Lemma 6.8 and Theorem 5.1, yields

$$
\log \tau_{M}\left(Y_{t}, \iota_{t}\right)=\tilde{I}\left(|t|^{-1}\right)+O\left(\log \log |t|^{-1}\right), \quad|\tilde{I}(T)| \leq C(\log T+1)
$$

where $\tilde{I}(T)=I(T)+\beta \log T$. Let $\eta(t)$ be a relative canonical form such that $\eta(t) \neq 0$. Let $\omega_{1}(t), \cdots, \omega_{g}(t)$ be a basis of $\pi_{*} \Omega_{Y / D}^{1}$. We may assume that $\omega_{1}(0)$ has at most logarithmic pole at $o$ and $\omega_{2}(0), \cdots, \omega_{g}(0)$ are holomorphic as $Z_{0}$ has only one node. By Theorem 3.5, one has the following on $D \backslash\{0\}$;

$$
\frac{i}{2 \pi} \bar{\partial} \partial\left\{\log \tau_{M}\left(Y_{t}, \iota_{t}\right)+\frac{r(M)-6}{8} \log \|\eta(t)\|^{2}+\frac{1}{2} \log \operatorname{det}\left(\int_{Z_{t}} \omega_{i}(t) \wedge \bar{\omega}_{j}(t)\right)\right\}=0 .
$$

Since $\log \|\eta(t)\|^{2}=O(1)$ and $\log \operatorname{det}\left(\int_{Z_{t}} \omega_{i}(t) \wedge \bar{\omega}_{j}(t)\right)=O\left(\log \log |t|^{-1}\right)$ as $t \rightarrow 0$, by the same argument as [B-B, Proposition 10.1] together with (6.35-36), there exits $\alpha \in \mathbb{R}$ such that

$$
\log \tau_{M}\left(Y_{t}, \iota_{t}\right)=\alpha \log |t|^{2}+O(1) \quad(t \rightarrow 0)
$$

By (6.35), $\alpha=\lim _{T \rightarrow \infty} \tilde{I}(T) / \log T$ is an invariant of the instanton $\left(X, \omega_{X}\right)$.

To determine $\alpha$, let us compute an example. Take $M=\mathrm{II}_{1,1} \oplus E_{8}(-2)$. Let $\Phi$ be Borcherds's $\Phi$-function of weight 12 over $\Omega$, the Hermitian domain of type IV associated to the lattice $\mathrm{II}_{1,1} \oplus \mathrm{II}_{1,1} \oplus \Lambda_{24}(-1)$, with zero divisor $\mathcal{D}$ (the discriminant locus) where $\Lambda_{24}$ is the 24-dimensional Leech lattice. (For $\Phi$, see [B2,3].). Put 
$\Psi_{M^{\perp}}:=\left.\Phi\right|_{\Omega_{M}}$ for the restriction. As the orthogonal compliment of $E_{8}(2)$ in $\Lambda_{24}$ is the 16-dimensional Barnes-Wall lattice $\Lambda_{16}$ ([B2], $[\mathrm{C}-\mathrm{S}]$ ) which is free from roots, $\Psi_{M}$ is a nonzero holomorphic modular from of weight 12 . Let $d \in \Delta\left(\mathrm{II}_{1,1} \oplus \mathrm{II}_{1,1} \oplus\right.$ $\Lambda_{24}(-1)$ ), and $\pi(d) \in \Lambda_{16}^{\vee}(-1)$ be the orthogonal projection. Then, $H_{d} \cap \Omega_{M} \neq \emptyset$ if and only if $-2<\langle\pi(d), \pi(d)\rangle \leq 0$. Computing $\theta_{\Lambda_{16}^{\vee}}(\tau)$ (cf. (8.19)), we find that there is no norm 1 or $3 / 2$ element in $\Lambda_{16}^{\vee}$, and thus $\pi(d)=0$. Namely, the zero divisor of $\Psi_{M \perp}$ coincides with $\mathcal{D}_{M}$. Since $j_{M}$ takes its value in $S^{2}\left(\mathcal{A}_{1}\right)$ by Theorem 2.5 and $j_{M}^{*}\left(\Delta_{1} \Delta_{2}\right)$ vanishes of order 2 along $\mathcal{D}_{M}$ (because the quotient map $\Omega_{M} \rightarrow \mathcal{M}_{M}$ blanches of order 2 along $\mathcal{D}_{M}$ ) where $\Delta_{i}$ is the Jacobi $\Delta$-function in the $i$-th variable, $\Delta^{\prime}:=\Psi_{M} \otimes j_{M}^{*}\left(\Delta_{1} \Delta_{2}\right)$ vanishes of order 3 along $\mathcal{D}_{M}$. As $\mathcal{D}_{M}$ is the divisor of type $(2,1)$ by Theorem 2.5 , it follows from Theorem 3.3 and (3.37) together with [B-B, Proposition 10.1] that

$$
\frac{i}{2 \pi i} \bar{\partial} \partial \log \left[\tau_{M}\left\|\Delta^{\prime}\right\|^{\frac{1}{12}}\right]=-\left(\alpha+\frac{1}{8}\right) \delta_{\mathcal{D}_{M}}
$$

outside of subvarieties of codimension $\geq 2$ where $\delta_{\mathcal{D}_{M}}$ is the current $\int_{\mathcal{D}_{M}}$. Since $\tau_{M}$ and $\left\|\Delta^{\prime}\right\|$ are $\Gamma_{M}$-invariant, (6.38) can be regarded as an equation of currents over $\mathcal{M}_{M}$ by Hartogus's extension theorem. From the residue theorem, it follows that $\alpha=-1 / 8$.

Corollary 6.1. If $\Lambda=\mathrm{II}_{1,1} \oplus E_{8}(-2)$, there exists a constant $C_{\Lambda} \neq 0$ such that $\tau_{\Lambda}=\left\|\Delta_{\Lambda}\right\|^{-1 / 4}$ where $\Delta_{\Lambda}=\left.C_{\Lambda} \Phi\right|_{\Omega_{\Lambda}} \otimes j_{\Lambda}^{*}\left(\Delta_{1} \Delta_{2}\right)$ and $\Phi$ is the denominator function of the fake monster Lie algebra.

\section{$\S 7$. Identification of $\tau_{M}$ with an Automorphic From}

Let $\mathcal{A}_{g}$ be the Siegel modular variety and $\mathcal{A}_{g}^{*}$ be the Satake compactification. Let $j_{M}: \Omega_{M} \rightarrow \mathcal{A}_{g(M)}$ be the rational map as in Proposition 2.4, and $\hat{\Omega}_{M}=$ $\overline{1 \times j_{M}\left(\Omega_{M}\right)}$ be the closure of the graph of $j_{M}$ in $\Omega_{M} \times \mathcal{A}_{g(M)}$. Let $p_{1}: \hat{\Omega}_{M} \rightarrow \Omega_{M}$ and $p_{2}: \hat{\Omega}_{M} \rightarrow \mathcal{A}_{g}$ be the morphisms induced by the projections. We regard $j_{M}^{*} \omega_{\mathcal{A}_{g}}$ (which is originally defined on $\Omega_{M}^{0}$ ) as a current on $\Omega_{M}^{0} \cup \mathcal{D}_{M}$ by putting $j_{M}^{*} \omega_{\mathcal{A}_{g}}=p_{1 *} p_{2}^{*} \omega_{\mathcal{A}_{g}}$ where $\mathcal{D}_{M}^{0}:=\bigcup_{\delta \in \Delta(N)} H_{\delta}^{0}\left(H_{\delta}^{0}=H_{\delta} \backslash \bigcup_{d \neq \pm \delta} H_{d}\right)$.

Theorem 7.1. One has the following equation of currents on $\Omega_{M}^{0} \cup \mathcal{D}_{M}^{0}$;

$$
\frac{i}{2 \pi} \bar{\partial} \partial \log \tau_{M}=\frac{1}{8} \delta_{\mathcal{D}_{M}}-\frac{r(M)-6}{8} \omega_{M}-\frac{1}{2} j_{M}^{*} \omega_{\mathcal{A}_{g(M)}}
$$

Proof. Theorems 3.5, 5.2, 6.1, together with Bismut-Bost's extension argument ([B-B, Proposition 10.2]), yields the assertion.

Let $\mathcal{F}_{g}(g=g(M))$ be the sheaf of Siegel modular forms of weight 1 over $\mathcal{A}_{g}$. Set $\lambda_{M}:=i_{*} \mathcal{O}_{\Omega_{M}}\left(\left(j_{M}^{0}\right)^{*} \mathcal{F}_{g}\right)$ where $i: \Omega_{M}^{0} \cup \mathcal{D}_{M}^{0} \hookrightarrow \Omega_{M}$ is the inclusion. It is an invertible sheaf on $\Omega_{M}$ because $j_{M}^{0}: \Omega_{M}^{0} \cup \mathcal{D}_{M}^{0} \rightarrow \mathcal{A}_{g}$ is regular and is defined out side of subvarieties of codimension 2. Since $j_{M}^{0}$ is independ of markings, $\lambda_{M}$ is invariant under $\Gamma_{M}$. Namely, it is a $\Gamma_{M}$-module. Let $\chi: \Gamma_{M} \rightarrow \mathbb{C}^{*}$ be a character. 
Definition 7.1. $f \in H^{0}\left(\Omega_{M}, \lambda_{M}^{\otimes q}\right)$ is said to be an automorphic form of weight $(p, q)$ with character $\chi$ if

$$
f(\gamma \cdot z)=\chi(\gamma) j(\gamma, z)^{p} \gamma^{*} f(z)
$$

for any $z \in \Omega_{M}$ and $\gamma \in \Gamma_{M}$ where $j(\gamma, z):=\left\langle\gamma \cdot z, l_{M}\right\rangle /\left\langle z, l_{M}\right\rangle$ is an automorphic factor. The Petersson norm, $\|f\|$, is defined by

$$
\|f(z)\|^{2}:=K_{M}(z, \bar{z})^{p} \operatorname{det} \operatorname{Im}\left(j_{M}(z)\right)^{q}|f(z)|^{2} .
$$

Here, $K_{M}(z, \bar{z})$ is the Bergman kernel and $l_{M} \in N_{\mathbb{C}}$ is the same vector as in (3.4).

In the sequel, we often omit character. Thus, an automorphic form is rigorously speaking an automorphic form with some character. Since $\log j_{M}^{*}\|\psi\|$ becomes a locally integrable function on $\Omega_{M}$ for any meromorphic Siegel modular form $\psi$, the curvature current of $\left(\lambda_{M},\|\cdot\|\right)(\|\cdot\|$ is the Petersson norm) can be defined in the usual manner, and coincides with $c_{1}\left(\lambda_{M},\|\cdot\|\right)=j_{M}^{*} \omega_{\mathcal{A}_{g}}$ on $\Omega_{M}^{0} \cup \mathcal{D}_{M}^{0}$.

Theorem 7.2. Suppose $r(M) \leq 17$. Then, there exists a modular form $\Delta_{M}$ of weight $(r(M)-6,4)$ such that $\tau_{M}=\left\|\Delta_{M}\right\|^{-1 / 4}$ and $\operatorname{div}\left(\Delta_{M}\right)=\mathcal{D}_{M}$.

Proof. Take a non-zero meromorphic modular form $\phi$ of weight $(r-6,4)$ such that $\mathcal{D}_{M}$ is not contained in the zero and polar locus of $\phi$. Put $F:=\tau_{M}^{8}\|\phi\|^{2}$. By Theorem 7.1 and Hartogus's theorem, one has $\frac{i}{2 \pi} \bar{\partial} \partial \log F=\delta_{\mathcal{D}_{M}}-\delta_{\operatorname{div}(\phi)}$ on $\Omega_{M}$. Therefore, $\partial \log F$ is a $\Gamma_{M}$-invariant meromorphic 1-form on $\Omega_{M}$ with at most logarithmic poles, and thus $G(y):=\exp \left(\int_{*}^{y} \partial \log F\right)\left(*\right.$ is a reference point in $\left.\Omega_{M}\right)$ is a meromorphic function on $\Omega_{M}$ such that $\operatorname{div}(G)=\mathcal{D}_{M}-\operatorname{div}(\phi)$. Let $\gamma \in \Gamma_{M}$. Let $[\gamma]$ be a simple closed real curve in $\mathcal{M}_{M}=\Omega_{M} / \Gamma_{M}$ corresponding to a path joining $y$ and $\gamma \cdot y$. As $\Omega_{M}$ is diffeomorphic to the cell, the homotopy class of $[\gamma]$ does not depend on a choice of $y$. Thus, $\chi(\gamma)=\exp \left(\int_{[\gamma]} \partial \log F\right)=G(\gamma \cdot y) / G(y)$ is independent of $y$ and becomes a character of $\Gamma_{M}$. Since $\Gamma_{M} /\left[\Gamma_{M}, \Gamma_{M}\right]$ is finite by Kazhdan's theorem $([\mathrm{Kz}]), \chi$ takes its values in $S^{1}=U(\mathbb{C})$. Therefore, $\log |G|^{2}$ is a $\Gamma_{M}$-invariant pluriharmonic function whose divisor is the same as $\log F$. Since the Satake-Baily-Borel boundary of $\mathcal{M}_{M}$ has codim $\geq 2$ when $r(M) \leq 17$, Hartogus's theorem implies that there exists a constant $C \neq 0$ such that $F=C^{2}|G|^{2}$. Thus, we get $\tau_{M}^{-8}=C^{2}|G|^{2}\|\phi\|^{2}$, and $\Delta_{M}:=C G \cdot \phi$ is the desired form.

Let $\delta \in \Delta(N)$. If $\langle M \oplus \delta\rangle$ denotes the smallest 2-elementary lattice generated by $M, \delta$, then $\Omega_{\langle M \oplus \delta\rangle}$ can naturally be identified with $H_{\delta}$. As $\Delta_{M}$ is a section of $\lambda_{M}^{\otimes 4}$ which vanishes of order one along $H_{\delta}$, it follows from Proposition 2.5 that $\Delta_{M} \cdot\left\langle z, l_{M}\right\rangle /\langle z, \delta\rangle$ restricted to $H_{\delta}^{0}$ is a section of $\lambda_{\langle M \oplus \delta\rangle}^{\otimes 4}$. Note that if $g(\langle M \oplus \delta\rangle)=$ $g(M)-1, \mathcal{A}_{g-1}$ is considered to be one of the boundary components of $\mathcal{A}_{g}^{*}$ and the restriction map $S: \mathcal{F}_{g}^{\otimes 4} \rightarrow \mathcal{F}_{g-1}^{\otimes 4}$ coincides with the Siegel operator $([\mathrm{F}])$.

Theorem 7.3. Under the identification $\Omega_{\langle M \oplus \delta\rangle}=H_{\delta}$, one has

$$
\Delta_{\langle M \oplus \delta\rangle}(y)=C_{\langle M \oplus \delta\rangle} \lim _{z \rightarrow y} \frac{\left\langle z, l_{M}\right\rangle}{\langle z, \delta\rangle} \Delta_{M}(z)=\left.C(M, \delta) \frac{\left\langle\cdot, l_{M}\right\rangle}{\langle\cdot, \delta\rangle} \Delta_{M}\right|_{H_{\delta}}(y) .
$$

Here $C_{\langle M \oplus \delta\rangle}$ is a nonzero constant.

Proof. Put $\delta:=\delta_{0}$. We separate the proof into two cases.

Case (1) Assume $\left\langle\delta_{0}, \delta_{1}\right\rangle=-1$ for some $\delta_{1} \in \Delta(N)$. With this condition, $M \oplus \mathbb{Z} \delta_{0}$ 
is primitive in $L_{K 3}$ and $\left\langle M \oplus \delta_{0}\right\rangle=M \oplus \mathbb{Z} \delta_{0}$ which implies $g\left(\left\langle M \oplus \delta_{0}\right\rangle\right)=g(M)-1$. By the condition, $\mathbb{Z} \delta_{0} \oplus \mathbb{Z} \delta_{1}=A_{2}(-1)$ where $A_{2}$ is the $A_{2}$-root lattice whose roots are $\pm \delta_{0}, \pm \delta_{1}, \pm \delta_{2}$ where $\delta_{2}:=\delta_{0}-\delta_{1}$.

Step 1. Take $\omega_{0} \in\left(H_{\delta_{0}} \cap H_{\delta_{1}}\right)^{0}:=H_{\delta_{0}} \cap H_{\delta_{1}} \backslash \cup_{d \neq \pm \delta_{i}} H_{d^{\prime}}$. Choose $\kappa_{0} \in C(M)$ such that $\left\langle\kappa_{0}, d\right\rangle \neq 0$ for any $d \in \Delta(M)$. By the surjectivity of the period map, there exists a marked $K 3$ surface $\left(X_{0}, \phi\right)$ with nef and big line bundle $L_{0}$ such that $\pi\left(X_{0}, \phi\right)=\omega_{0}$ and $\phi\left(c_{1}\left(L_{0}\right)\right)=\kappa_{0}$. Choosing $0<\epsilon \ll 1$, we may suppose that there exists a Kähler class whose image by $\phi$ is $\kappa:=\kappa_{0}-\epsilon \delta_{0}$. Let $C_{\delta_{i}}$ be the cycle corresponding to $\delta_{i}$. As $\left\langle\kappa, \delta_{i}\right\rangle>0$, any $C_{\delta_{i}}$ is effective. We choose $\epsilon$ so small that $C_{\delta_{1}}, C_{\delta_{2}}$ are irreducible -2 -curves and $C_{\delta_{0}}=C_{\delta_{1}} \cup C_{\delta_{2}}$.

Let $U$ be a small neighborhood of $\left(X_{0}, \phi\right)$ in $\tilde{\Omega}_{M}$, and $(X, \phi) \rightarrow U$ be the universal family. By construction, there exists a relatively nef and big line bundle $L \rightarrow X$ which restricted to $X_{0}$ is $L_{0}$. Choosing $m \gg 1$, let $\Phi_{|m L|}: X \rightarrow \mathbb{P}^{N}$ be the morphism associated to the complete linear system $|m L|$, and $Y:=\Phi_{|m L|}(X) \rightarrow U$ be the image. As $\kappa_{0}$ does not intersect $\delta_{i}$, the cycle $C_{\delta_{i}}$ corresponding to $\delta_{i}$ is an irreducible -2-curve in $X_{t}$ if $t \in H_{\delta_{i}}^{0}$ (cf. Lemma 2.3). By above construction, $C_{\delta_{0}}$ splits into two components if $t \in\left(H_{\delta_{0}} \cap H_{\delta_{1}}\right)^{0} ; C_{\delta_{0}}=C_{\delta_{1}} \cup C_{\delta_{2}}$. By Mayer's theorem, $\Phi_{|m L|}: X_{t} \rightarrow Y_{t}$ is the minimal resolution whose exceptional locus is $C_{\delta_{i}}$ if $t \in H_{i}^{0}(i=0,1,2), C_{\delta_{0}}=C_{\delta_{1}} \cup C_{\delta_{2}}$ if $t \in\left(H_{\delta_{0}} \cap H_{\delta_{1}}\right)^{0}$, and empty if outside of the discriminant locus. Put $o \in Y_{0}$ for the image of $C_{\delta_{0}}$. As $\mathbb{Z} \delta_{1} \oplus \mathbb{Z} \delta_{2}$ is the $A_{2}$-root system, $\left(Y_{0}, o\right)$ is a $K 3$ surface with a $A_{2}$-singularity. Set

$\mathcal{M}_{A_{2}}:=\left\{\alpha \in \mathbb{C}^{3} ; \sum \alpha_{i}=0\right\}, \quad Z:=\left\{(x, \alpha) \in \mathbb{C}^{3} \times \mathcal{M}_{A_{2}} ; x_{1} x_{2}-\prod\left(x_{3}+\alpha_{i}\right)=0\right\}$.

Consider the deformation of $A_{2}$-singularity $(Z, 0) \rightarrow\left(\mathcal{M}_{A_{2}}, 0\right)$ on which acts $S_{3}=$ $W\left(A_{2}\right)$ by the permutation of coordinates $\alpha$. Then, $\left(Z / S_{3}, 0\right) \rightarrow\left(\mathcal{M}_{A_{2}} / S_{3}, 0\right)$ is the semiuniversal deformation of $A_{2}$-singularity. The discriminant locus of $Z \rightarrow \mathcal{M}_{A_{2}}$ is $D=D_{0} \cup D_{1} \cup D_{2}$ where $D_{i}=\left\{\alpha \in \mathcal{M}_{A_{2}} ; \alpha_{k}-\alpha_{j}=0\right\} \quad(\{i, j, k\}=\{0,1,2\})$. By the versality, there exist maps $F:(Y, o) \rightarrow(Z, 0)$ and $f:(U, 0) \rightarrow\left(\mathcal{M}_{A_{2}}, 0\right)$ which commute with the projections such that $(Y, o)=F^{*}(Z, 0)$ and $f\left(H_{\delta_{i}}\right)=D_{i}$.

Let $\phi^{\prime}=I_{M} \circ \phi$ (cf. (2.6)) be another marking, and $\left(X^{\prime}, \phi^{\prime}\right) \rightarrow U^{\prime}$ be the universal family such that $U^{\prime}=I \circ U$ (cf. (2.8)). Let $L^{\prime} \rightarrow X^{\prime}$ be the relatively nef and big line bundle such that $\phi^{\prime}\left(c_{1}\left(L_{s}^{\prime}\right)\right)=\kappa_{0}$. By the similar construction as before, we get a family $Y^{\prime} \rightarrow U^{\prime}$ such that $X^{\prime} \rightarrow Y^{\prime}$ is the simultaneous resolution. Since $\phi^{-1}\left(\kappa_{0}\right)$ and $\left(\phi^{\prime}\right)^{-1}\left(\kappa_{0}\right)$ are weak polarizations ([Mo, pp.318]) of $\left(X_{t}, \phi\right)$ and $\left(X_{t}^{\prime}, \phi^{\prime}\right)$ respectively, we can define the weakly polarized period map ([Mo, pp.318]) $U \rightarrow V$ and $U^{\prime} \rightarrow V$ by sending $\left(\left[\omega_{t}\right], \kappa_{t}\right)$ to $\left(\left[\omega_{t}\right], \kappa_{0}\right)$ where $\left[\omega_{t}\right]$ is the period and $\kappa_{t}$ is the Kähler class. Note that the weak polarized period domain in our situation is $\Omega_{M} \times C(M)^{+}$and $V$ is its subset. As $U \rightarrow V$ (resp. $U^{\prime} \rightarrow V$ ) is an isomorphism, we may regard $Y$ and $Y^{\prime}$ are families over $V$. Since there exists the universal marked family of generalized $K 3$ surfaces over $\Omega_{M} \times C(M)^{+}([\mathrm{Mo}$, pp.321]), we get an identification $e: Y^{\prime} \cong Y$. Let $p: X \rightarrow Y$ and $p^{\prime}: X^{\prime} \rightarrow Y^{\prime}$ be the simultaneous resolution, and $\iota_{M}: X \rightarrow X^{\prime}$ be the isomorphism as in (2.8). Then, $\iota:=e \circ p^{\prime} \circ \iota_{M} \circ p^{-1}$ is a rational automorphism over $Y$. By the weakly polarized global Torelli theorem ([Mo, pp.319]), $\iota_{t}$ is an anti-symplectic involution on $Y_{t}$ for any $t \in V$ and $\iota$ is holomorphic everwhere. By appropriate normalizations, we may suppose $(Y, o)=(Z, 0)$ and $\iota\left(x_{0}, x_{1}, x_{2}\right)=\left(x_{1}, x_{0}, x_{2}\right)$ in $(7.1)$.

Step 2. Let $C \rightarrow V$ be the family of fixed curves. By Theorem 2.5, we get the decomposition $C=C^{(g)}+\sum E_{i}$ where $C_{t}^{(g)}$ is a smooth irreducible curve of genus 
$g=g(M)$ for generic $t \in V$ and $E_{i}$ is a family of smooth rational curves. As $C_{0}^{(g)}$ is a fixed locus, $\operatorname{Sing} C_{0}^{(g)}=\operatorname{Sing} Y_{0}=o$. Moreover it is a Cartier divisor because $\left(C_{0}^{(g)}, o\right)=\left(Z_{0}, 0\right) \cap\left\{x_{0}-x_{1}=0\right\}=\left\{\left(x_{2}, x_{3}\right) ; x_{2}^{2}-x_{3}^{3}=0\right\}$. As $C_{0}^{(g)} \cdot C_{0}^{(g)}=$ $2(g-1) \geq 0, C_{0}^{(g)}$ is a nef and effective divisor. Since $\left(Y_{0}, o\right)$ is an $A_{2}$-singularity, $o$ can not belong to the fixed part of the linear system $\left|C_{0}^{(g)}\right|$ by Saint-Donat's theorem. Therefore, we can pick up $f_{1}, \cdots, f_{g}$, nonconstant meromorphic functions on $Y$, such that $\operatorname{div}\left(f_{i}\right)=P_{i}-C^{(g)}$ with $o \notin P_{g}$ but $\operatorname{Sing} Y_{t} \in P_{i}$ for $1 \leq i<g$. Let $\omega_{Y / V}$ be a nowhere vanishing relative 2-form on $Y$. Let $\varphi_{i}(t)$ be the 1-form on $C_{t}^{(g)}$ defined by $\varphi_{i}(t):=\operatorname{Res}_{C_{t}^{(g)}} f_{i} \omega_{Y / V}$. As $\operatorname{Sing} Y_{t}$ is contained in the zero locus of $f_{j}$ for $j<g, \varphi_{j}(t)(j<g)$ becomes a holomorphic 1-form on the normalization of $C_{t}^{(g)}$ when $t \in H_{\delta_{0}}$. Thus, if $j<g$,

$$
\int_{C_{t}^{(g)}} \varphi_{i}(t) \wedge \overline{\varphi_{j}(t)} \rightarrow \int_{C_{0}^{(g)}} \varphi_{i}(0) \wedge \overline{\varphi_{j}(0)}<\infty \quad\left(t \rightarrow H_{\delta_{0}}\right)
$$

Let $A_{1}, B_{1}, \cdots, A_{g}, B_{g}$ be the symplectic basis of $H^{1}\left(C_{t}^{(g)}, \mathbb{Z}\right)$. As $\left(C_{0}^{(g)}, 0\right)$ is the cusp, we may assume that $A_{g}$ and $B_{g}$ are vanishing cycles, and all other cycles converges to the symplectic basis of the normalization of $C_{0}^{(g)}$. Since we have fixed the symplectic basis, $j_{M}$ takes its value in $\mathfrak{S}_{g}$ :

$$
\tau\left(j_{M}(t)\right)=\left(\int_{A_{i}} \varphi_{j}(t)\right)^{-1}\left(\int_{B_{i}} \varphi_{j}(t)\right) \in \mathfrak{S}_{g} .
$$

As the bidegree $(1,1)$ part of the diagonal in $C_{t}^{(g)} \times C_{t}^{(g)}$ is homologous to $\sum A_{i}^{(1)} B_{i}^{(2)}-$ $B_{i}^{(1)} A_{i}^{(2)}$ in $H^{2}\left(C_{t}^{(g)} \times C_{t}^{(g)} ; \mathbb{Z}\right)$, it follows from (7.2) that

$$
\int_{C_{t}^{(g)}} \varphi_{g}(t) \overline{\varphi_{g}(t)}=\operatorname{Im} \tau_{g g}\left(j_{M}(t)\right)\left|\int_{A_{g}} \varphi_{g}(t)\right|^{2}+O(1) .
$$

Let $E_{\beta}:=\left\{\left(x_{2}, x_{3}\right) ; x_{2}^{2}=\left(x_{3}+1\right)\left(x_{3}+\beta\right)\left(x_{3}-1-\beta\right)\right\}$ be an elliptic curve and $\gamma_{1}, \gamma_{2}$ be the symplectic basis such that $\gamma_{1}$ converges to the cycle $\left|x_{3}+1\right|=\epsilon$ as $\beta \rightarrow 0$. Since $o \notin P_{g}$, multiplying a constant if necessary, we may assume $f_{g}=1 /\left\{x_{2}^{2}-\prod\left(x_{3}+\alpha_{i}\right)\right\}+O(1)$ on a neighborhood of $o$. When $t \rightarrow H_{\delta_{0}}, \alpha(t) \rightarrow D_{0}$ and $\alpha_{2}(t) \rightarrow \alpha_{1}(t)$. Putting $\beta=\alpha_{1} / \alpha_{2}$, we may suppose $A_{g}$ is identified with $\gamma_{1}$ and get

$$
\begin{aligned}
\int_{A_{g}} \varphi_{g}(t) & =\int_{A_{1}} \frac{d z}{\sqrt{\left(z+\alpha_{0}\right)\left(z+\alpha_{1}\right)\left(z+\alpha_{2}\right)}}+O(1) \\
& =\alpha_{2}^{-\frac{1}{2}} \int_{\gamma_{1}} \frac{d z}{\sqrt{(z+1)(z+\beta)(z-1-\beta)}}+O(1)=\frac{2 \pi}{\sqrt{3}} \alpha_{2}^{-\frac{1}{2}}+O(1) .
\end{aligned}
$$

Let $W$ be a small neighborhood of $\omega_{0} \in\left(H_{\delta_{0}} \cap H_{\delta_{1}}\right)^{0}$ in $H_{\delta_{0}}^{0}$. Since

$$
\left.\left[\frac{\operatorname{det}\left(\int_{C^{(g)}} \varphi_{i} \overline{\varphi_{j}}\right)}{\int_{C^{(g)}} \varphi_{g} \overline{\varphi_{g}}}\right]\right|_{W}=\left.\operatorname{det}\left(\int_{C^{(g)}} \varphi_{i} \overline{\varphi_{j}}\right)_{i, j<g}\right|_{W}
$$


by $(7.2-5)$, it follows that

$$
\begin{aligned}
& \frac{i}{2 \pi} \bar{\partial} \partial\left[\left.\log \frac{\operatorname{det}\left(\int_{C^{(g)}} \varphi_{i} \overline{\varphi_{j}}\right)}{j_{M}^{*} \operatorname{Im} \tau_{g g}}\right|_{W}\right] \\
& =\frac{i}{2 \pi} \bar{\partial} \partial\left[\log \left|\int_{A_{g}} \varphi_{g}\right|^{2}||_{W}\right]+\frac{i}{2 \pi} \bar{\partial} \partial\left[\left.\log \operatorname{det}\left(\int_{C^{(g)}} \varphi_{i} \overline{\varphi_{j}}\right)_{i, j<g}\right|_{W}\right] \\
& =\frac{1}{2} \delta_{H_{\delta_{0}} \cap H_{\delta_{1}} \cap W}+\left.j_{\left\langle M \oplus \delta_{0}\right\rangle}^{*} \omega_{\mathfrak{S}_{g-1}}\right|_{W} .
\end{aligned}
$$

Since $H_{d} \cap H_{\delta_{0}} \cap W \neq \emptyset(d \in \Delta(N))$ iff $d= \pm \delta_{0}, \pm \delta_{1}, \pm \delta_{2}$, it follows from Theorem 7.1 that

$$
\begin{aligned}
& \frac{i}{2 \pi} \bar{\partial} \partial \log \left[\left.\tau_{M} \operatorname{det}\left(\int_{C^{(g)}} \varphi_{i} \overline{\varphi_{j}}\right)^{\frac{1}{2}}\left(\frac{\left|\left\langle w, \delta_{0}\right\rangle\right|^{2}}{\langle w, \bar{w}\rangle}\right)^{\frac{1}{8}}\right|_{W}\right] \\
& =\frac{1}{8} \sum_{d \in \Delta(N) \backslash\left\{ \pm \delta_{0}\right\} / \pm 1} \delta_{H_{d} \cap H_{\delta_{0}} \cap W}-\frac{r(M)-5}{8} \omega_{\left\langle M \oplus \delta_{0}\right\rangle} \\
& =\frac{1}{4} \delta_{H_{\delta_{0}} \cap H_{\delta_{1}} \cap W}-\frac{r\left(\left\langle M \oplus \delta_{0}\right\rangle\right)-6}{8} \omega_{\left\langle M \oplus \delta_{0}\right\rangle}
\end{aligned}
$$

which, together with (7.7), yields

$$
\begin{aligned}
& \frac{i}{2 \pi} \bar{\partial} \partial \log \left[\left.\tau_{M}\left(j_{M}^{*} \operatorname{Im} \tau_{g g}\right)^{\frac{1}{2}}\left(\frac{\left|\left\langle w, \delta_{0}\right\rangle\right|^{2}}{\langle w, \bar{w}\rangle}\right)^{\frac{1}{8}}\right|_{W}\right] \\
& =-\frac{r\left(\left\langle M \oplus \delta_{0}\right\rangle\right)-6}{8} \omega_{\left\langle M \oplus \delta_{0}\right\rangle}-\left.\frac{1}{2} j_{\left\langle M \oplus \delta_{0}\right\rangle}^{*} \omega_{\mathfrak{S}_{g-1}}\right|_{W} .
\end{aligned}
$$

Step 3. Let $\delta_{3} \in \Delta(N)$ such that $\left\langle\delta_{3}, \delta_{0}\right\rangle=0$. Take $\omega_{0} \in\left(H_{\delta_{0}} \cap H_{\delta_{3}}\right)^{0}$ and choose $\kappa_{0} \in C_{M}$ as in Step 1. Similarly as before, there exists a marked $K 3$ surface $\left(X_{0}, \phi\right)$ with nef and big line bundle $L_{0}$ such that $\pi\left(X_{0}, \phi\right)=\omega_{0}$ and $\phi\left(c_{1}\left(L_{0}\right)\right)=\kappa_{0}$. Let $C_{\delta_{i}}$ be the cycle corresponding to $\delta_{i}$. Then, $C_{\delta_{0}}$ and $C_{\delta_{4}}$ are mutually disjoint irreducible -2-curves. Let $U$ be a small neighborhood of $\left(X_{0}, \phi\right)$ in $\tilde{\Omega}_{M},(X, \phi) \rightarrow U$ the universal family, and $L \rightarrow X$ the relatively nef and big line bundle whose restriction to $X_{0}$ is $L_{0}$. Using $|m L|$, set $Y:=\Phi_{|m L|}(X) \rightarrow U$ for $m \gg 1$. As $\left\langle\kappa_{0}, \delta_{i}\right\rangle=0$, the cycle $C_{\delta_{i}}$ corresponding to $\delta_{i}$ is an irreducible -2-curve in $X_{t}$ if $t \in H_{\delta_{i}}^{0}$. Thus, $\Phi_{|m L|}: X_{t} \rightarrow Y_{t}$ is the minimal resolution whose exceptional locus is $C_{\delta_{i}}$ if $t \in H_{i}^{0}(i=0,3), C_{\delta_{0}} \cup C_{\delta_{3}}$ if $t \in\left(H_{\delta_{0}} \cap H_{\delta_{3}}\right)^{0}$, and empty if outside of the discriminant locus. Put $o_{i}$ for the image of $C_{\delta_{i}}$. By construction, $\left(Y_{0}, o_{0}, o_{3}\right)$ is a $K 3$ surface with two $A_{1}$-singularities. Let $\iota: Y \rightarrow Y$ be the anti-symplectic involution constructed as before, and $C^{(g)} \rightarrow U$ be the family of fixed curves of maximal genus. By construction, $C^{(g)} \rightarrow U$ is f.s.o. in the sense of [B-B] (though the total space admits two nodes here). Since $C_{t}^{(g)}$ has a node for $t \in H_{\delta_{0}}^{0}$ (because $\left.g\left(\left\langle M \oplus \delta_{0}\right\rangle\right)=g(M)-1\right)$, we find $o_{0} \in C_{0}^{(g)}$. Let $\varphi_{1}(t), \cdots, \varphi_{g}(t)$ be a basis of relative 1 -forms for $C \rightarrow U$ such that $\varphi_{g}(t)$ has a logarithmic pole at $\operatorname{Sing} C_{t}^{(g)}$ if $t \in H_{\delta_{0}}$ and that $\varphi_{1}(t), \cdots, \varphi_{g-2}(t)$ are regular on the normalization of $C_{t}^{(g)}$ for 
any $t \in U$. If $o_{3} \in C_{0}^{(g)}$, we may suppose that $\varphi_{g-1}(0)$ has a logarithmic pole at $o_{3}$ and $\varphi_{g-1}(t)$ is regular on the normalization of $C_{0}^{(g)}$ for $t \in H_{\delta_{0}}^{0}$. If $o_{3} \notin C_{0}^{(g)}$, we may assume that $\varphi_{g-1}(t)$ is regular on the normalization of $C_{t}^{(g)}$ for any $t \in U$. Let $W$ be a small neighborhood of $\omega_{0} \in\left(H_{\delta_{0}} \cap H_{\delta_{3}}\right)^{0}$ in $H_{\delta_{0}}^{0}$. From [B-B, Propositon 13.3] together with Proposition 2.5, it follows that

$$
\begin{aligned}
\frac{i}{2 \pi} \bar{\partial} \partial\left[\left.\log \frac{\operatorname{det}\left(\int_{C^{(g)}} \varphi_{i} \overline{\varphi_{j}}\right)}{j_{M}^{*} \operatorname{Im} \tau_{g g}}\right|_{W}\right] & =\frac{i}{2 \pi} \bar{\partial} \partial\left[\left.\log \operatorname{det}\left(\int_{C^{(g)}} \varphi_{i} \overline{\varphi_{j}}\right)_{i, j<g}\right|_{W}\right] \\
& =\left.j_{\left\langle M \oplus \delta_{0}\right\rangle}^{*} \omega_{\mathfrak{S}_{g-1}}\right|_{W} .
\end{aligned}
$$

Since $H_{d} \cap H_{\delta_{0}} \cap W \neq \emptyset(d \in \Delta(N))$ iff $d= \pm \delta_{0}, \pm \delta_{3}$, comparing (7.8) and (7.10), we get

$$
\begin{aligned}
& \frac{i}{2 \pi} \bar{\partial} \partial \log \left[\left.\tau_{M}\left(j_{M}^{*} \operatorname{Im} \tau_{g g}\right)^{\frac{1}{2}}\left(\frac{\left|\left\langle w, \delta_{0}\right\rangle\right|^{2}}{\langle w, \bar{w}\rangle}\right)^{\frac{1}{8}}\right|_{W}\right] \\
& =\frac{1}{8} \delta_{H_{\delta_{0}} \cap H_{\delta_{3}} \cap W}-\frac{r\left(\left\langle M \oplus \delta_{0}\right\rangle\right)-6}{8} \omega_{\left\langle M \oplus \delta_{0}\right\rangle}-\left.\frac{1}{2} j_{\left\langle M \oplus \delta_{0}\right\rangle}^{*} \omega_{\mathfrak{S}_{g-1}}\right|_{W} .
\end{aligned}
$$

Step 4. In view of the proof of Theorem 7.2 , we may write $\Delta_{M}=\psi \otimes j_{M}^{*} E$ where $\psi$ is a meromorphic modular form over $\Omega_{M}$ of weight $g(r-6)$ and $E$ is an Eisenstein series of weight $4 g([\mathrm{~F}])$ whose divisor does not contain the boundary component $\mathcal{A}_{g}^{*} \backslash \mathcal{A}_{g}$. For $\tau=\left(\tau_{i j}\right)_{i, j \leq g} \in \mathfrak{S}_{g}$, write $\tau^{\prime}=\left(\tau_{i j}\right)_{i, j<g} \in \mathfrak{S}_{g-1}$. Let $S: H^{0}\left(\mathcal{A}_{g}, \mathcal{F}_{g}^{k}\right) \rightarrow H^{0}\left(\mathcal{A}_{g-1}, \mathcal{F}_{g-1}^{k}\right)$ be the Siegel operator. Since $j_{M}\left(H_{\delta_{0}}\right) \subset \mathcal{A}_{g-1}$, it follows from Proposition 2.5 that

$$
\left.\left(\frac{\left\langle\cdot, l_{M}\right\rangle}{\left\langle\cdot, \delta_{0}\right\rangle} \Delta_{M}\right)^{g}\right|_{H_{\delta_{0}}}=\left.\left(\frac{\left\langle\cdot, l_{M}\right\rangle}{\left\langle\cdot, \delta_{0}\right\rangle}\right)^{g} \psi\right|_{H_{\delta_{0}}} \otimes j_{\left\langle M \oplus \delta_{0}\right\rangle}^{*} S(E) .
$$

From the definition of Petersson norm, it follows that

$$
\begin{aligned}
\left\|\left.\left(\frac{\left\langle\cdot, l_{M}\right\rangle}{\left\langle\cdot, \delta_{0}\right\rangle} \Delta_{M}\right)^{g}\right|_{H_{\delta_{0}}}\right\|^{2}(w) & =\left.K_{M}^{-g(r-5)}\left|\left(\frac{\left\langle z, l_{M}\right\rangle}{\left\langle z, \delta_{0}\right\rangle}\right)^{g} \psi\right|_{z=w}\right|^{2} j_{M}^{*}\left(\operatorname{det} \operatorname{Im} \tau^{\prime}\right)^{4 g}|S(E)|^{2} \\
& =\left.\left\|\Delta_{M}\right\|^{2 g}\left(j_{M}^{*} \operatorname{Im} \tau_{g g}\right)^{-4 g}\left(\frac{\left|\left\langle z, \delta_{0}\right\rangle\right|^{2}}{\langle z, \bar{z}\rangle}\right)^{-g}\right|_{z=w} \\
& =\left.\tau_{M}^{-8 g}\left(j_{M}^{*} \operatorname{Im} \tau_{g g}\right)^{-4 g}\left(\frac{\left|\left\langle z, \delta_{0}\right\rangle\right|^{2}}{\langle z, \bar{z}\rangle}\right)^{-g}\right|_{z=w}
\end{aligned}
$$

where $K_{M}$ is the Bergman kernel as in (3.4). Comparing (7.9) and (7.11) with (7.13), $\left\|\Delta_{M} \cdot\left\langle\cdot, l_{M}\right\rangle /\left.\left\langle\cdot, \delta_{0}\right\rangle\right|_{H_{\delta_{0}}}\right\|^{2}$ satisfies the same $\bar{\partial} \partial$-equation as $\left\|\Delta_{\left\langle M \oplus \delta_{0}\right\rangle}\right\|^{2}$ over $\Omega_{\left\langle M \oplus \delta_{0}\right\rangle}^{0} \cup \mathcal{D}_{\left\langle M \oplus \delta_{0}\right\rangle}^{0}$. In view of the proof of Theorem 7.2, solution of the $\bar{\partial} \partial$-equation in Theorem 7.1 is unique up to constant. Thus, we get the assertion.

Case (2) Suppose that there is no $d \in \Delta(N)$ such that $\left|\left\langle d, \delta_{0}\right\rangle\right|=1$. Thus, for any $d \in \Delta(N), H_{d} \cap H_{\delta_{0}} \neq \emptyset$ iff $\langle d, \delta\rangle=0$. Now, we can prove the assertion in the same way as Step 3 and 4 in Case (1), and details are left to the reader. 


\section{$\S 8$. Borcherds's Products Arizing from $\Delta_{M}$}

8.1 Borcherds's Product. For a lattice $M$ of signature $\left(2, b^{-}\right)$, let $\left\{e_{\gamma}\right\}_{\gamma \in M^{\vee} / M}$ be the standard unitary basis of the group ring $\mathbb{C}\left[M^{\vee} / M\right]$. We denote by $\rho_{M}$ the Weil representation of the metaplectic group $M p_{2}(\mathbb{Z})$;

$$
\rho_{M}(T) e_{\gamma}=e^{\pi i\langle\gamma, \gamma\rangle} e_{\gamma}, \quad \rho_{M}(S) e_{\gamma}=\frac{\sqrt{i}^{b^{-}-2}}{\sqrt{\left|M^{\vee} / M\right|}} \sum_{\delta \in M^{\vee} / M} e^{2 \pi i\langle\gamma, \delta\rangle} e_{\delta}
$$

where $T=\left(\left(\begin{array}{ll}1 & 1 \\ 0 & 1\end{array}\right), 1\right), S=\left(\left(\begin{array}{cc}0-1 \\ 1 & 0\end{array}\right), \sqrt{\tau}\right)$ are the generators of $M p_{2}(\mathbb{Z})$. In this section, we suppose $M=\mathrm{II}_{1,1}(N) \oplus K(N=1,2$ and $K$ is a hyperbolic 2-elementary lattice.) Let $F(\tau)=\sum_{\gamma \in M^{\vee} / M} e_{\gamma} \sum_{k \in \mathbb{Q}} c_{\gamma}(k) q^{k}$ be a nearly holomorphic modular form of type $\rho_{M}$ with weight $1-b^{-} / 2$ which has the integral Fourier coefficients at the cusp. We denote by $\Psi_{M}(z, F)$ Borcherds's product attached to $F(\tau)$ ([Bo5]);

$-\log \left\|\Psi_{M}(z, F)\right\|^{4}=\int_{S L_{2}(\mathbb{Z}) \backslash \mathbb{H}} \bar{\Theta}_{M}(\tau ; z) F(\tau) y d x d y / y^{2}+c_{0}(0)\left(\Gamma^{\prime}(1)+2 \log \sqrt{2 \pi}\right)$.

$\Psi_{M}(z, F)$ is a function on the Grassmannian $G\left(2, b^{-}\right)$which is isomorphic to the tube domain $K_{\mathbb{R}}+\sqrt{-1} C(K)^{+}$. The following theorem is due to Borcherds.

\section{Theorem 8.1 ([Bo5, Theorem 13.3]).}

(1) $\Psi_{M}(z, F)$ is an automorphic form on $G\left(2, b^{-}\right)$for some arithmetic subgroup of $O(M)$ of weight $c_{0}(0) / 2$.

(2) The zeros or poles of $\Psi_{M}(z, F)$ lies on the divisor $\lambda^{\perp}\left(\lambda \in M, \lambda^{2}<0\right)$ of order $\sum_{0<x \in \mathbb{R}, x \lambda \in M^{\vee}} c_{x \lambda}\left(x^{2} \lambda^{2} / 2\right)$.

(3) $\Psi_{M}(z, F)$ admits the following holomorphic infinite product expansion near the cusp and $z \in K_{\mathbb{R}}+\sqrt{-1} W$;

$$
\Psi_{M}(z, F)=e^{2 \pi i\left\langle\rho\left(K, W, F_{K}\right), z\right\rangle} \prod_{\lambda \in K^{\vee} \cap W^{\vee}} \prod_{n \in \mathbb{Z} / N \mathbb{Z}}\left(1-e^{2 \pi i\langle\lambda, z+n / N\rangle}\right)^{c_{\lambda+n f^{\prime} / N}\left(\lambda^{2} / 2\right)}
$$

where $\rho\left(K, W, F_{K}\right)$ is the Weyl vector, $W$ is a Weyl chamber, $W^{\vee}$ the dual cone of $W$, and $f, f^{\prime}$ are the generators of $\mathrm{II}_{1,1}(N)$ such that $f \cdot f=f^{\prime} \cdot f^{\prime}=0, f \cdot f^{\prime}=N$.

8.2 2-Elementary $K 3$ Surfaces with $(g, \delta)=(0,1)$. By Nikulin's table ([Ni4]), all the primitive 2-elementary hyperbolic lattices in $L_{K 3}$ with $(g, \delta)=(0,1)$ are isometric to one of the following $S_{k}(1 \leq k \leq 9)$.

Let $h, \delta_{0}, \cdots, \delta_{8}$ be the basis of $\mathrm{I}_{1,9}(2)$ such that $h^{2}=2, h \cdot \delta_{i}=0, \delta_{i} \cdot \delta_{j}=-2 \delta_{i j}$. Then, $\mathrm{I}_{1,8}(2)=A_{1} \oplus A_{1}(-1)^{\oplus 8}=\mathbb{Z} h \oplus \mathbb{Z} \delta_{1} \oplus \cdots \oplus \mathbb{Z} \delta_{8}=\mathrm{I}_{1,9}(2) \cap \delta_{0}^{\perp}$. Put

$$
\rho:=\left(3 h-\delta_{0}-\cdots-\delta_{8}\right) / 2 \in \mathrm{I}_{1,9}(2)^{\vee}, \quad \kappa:=3 h-\left(\delta_{1}+\cdots+\delta_{8}\right) .
$$

$\Lambda$ is a sublattice of $\mathrm{I}_{1,9}(2)^{\vee}$ defined by $\Lambda=\mathbb{Z} \rho \oplus \mathbb{Z} \delta_{0} \oplus \rho^{\perp} \cap \delta_{0}^{\perp} \cap \mathrm{I}_{1,9}(2)$. Note that $\kappa^{\perp} \cap \mathrm{I}_{1,8}(2) \cong E_{8}(-2)$ (cf. [Man]). As $\delta_{0}^{\perp} \cap \Lambda=\mathbb{Z} h \oplus \mathbb{Z} \delta_{1} \oplus \cdots \oplus \mathbb{Z} \delta_{8}=\mathrm{I}_{1,8}(2)$, above $\delta_{0}, \cdots, \delta_{8}$ satisfy

$$
\delta_{0}^{\perp} \cap \cdots \cap \delta_{k-1}^{\perp} \cap \Lambda=\mathbb{Z} h \oplus \mathbb{Z} \delta_{k} \oplus \cdots \oplus \mathbb{Z} \delta_{8}=\mathrm{I}_{1,9-k}(2), \quad \delta_{i} \cdot \rho=1 .
$$


Define 2-elementary lattices $\Lambda_{k}, S_{k}, T_{k}(1 \leq k \leq 9)$ by $(8.5)$

$$
\Lambda_{k}:=\delta_{0}^{\perp} \cap \cdots \cap \delta_{k-1}^{\perp}=\mathrm{I}_{1,9-k}(2), \quad T_{k}:=\mathrm{II}_{1,1}(2) \oplus \Lambda_{k} \cong \mathrm{I}_{2,10-k}(2), \quad S_{k}:=T_{k}^{\perp} .
$$

Here, the first orthogonal compliment is considered in $\Lambda_{k}$, and the last one in $L_{K 3}$. Then, $\left(r\left(S_{k}\right), l\left(S_{k}\right), \delta\left(S_{k}\right)\right)=(10+k, 12-k, 1)$. Let $\pi_{k}: \Lambda \rightarrow \Lambda_{k}^{\vee}$ be the orthogonal projection. As is well known (cf. [Man]), the Weyl vector of $\Lambda_{k}$ is given by

$$
\rho_{k}:=\pi_{k}(\rho)=\left(3 h-\delta_{k}-\cdots-\delta_{8}\right) / 2 .
$$

We denote by $\operatorname{Am}\left(\Lambda_{k, \mathbb{R}}\right)$ the Weyl chamber containing $\rho_{k}$ and by $\operatorname{NE}\left(\Lambda_{k, \mathbb{R}}\right)$ the dual cone of $\operatorname{Am}\left(\Lambda_{k, \mathbb{R}}\right)$ i.e., $\operatorname{NE}\left(\Lambda_{k, \mathbb{R}}\right)=\left\{r \in \Lambda_{\mathbb{R}} ;\left\langle r, \operatorname{Am}\left(\Lambda_{k, \mathbb{R}}\right)\right\rangle>0\right\}$.

Put $M:=T_{k}=\mathrm{II}_{1,1}(2) \oplus \mathrm{I}_{1,9-k}(2)$. Vectors in $M$ are denoted by $(m, n, \lambda)$ $\left(m, n \in \mathbb{Z}, \lambda \in \mathrm{I}_{9-k}(2)\right)$ whose norm is $4 m n-\langle\lambda, \lambda\rangle$. The period domain $\Omega_{S_{k}}$ is isomorphic to the tube domain $\Lambda_{k, \mathbb{R}}+\sqrt{-1} C\left(\Lambda_{k}\right)$ via the following map;

$$
\Lambda_{k, \mathbb{R}}+\sqrt{-1} C\left(\Lambda_{k}\right)^{ \pm} \ni v \rightarrow(1 / 2,-\langle v, v\rangle / 2, v) \in \Omega_{S_{k}}^{ \pm} .
$$

We define $e_{0}, e_{1}, v_{0}, v_{1}, v_{2}, v_{3} \in \mathbb{C}\left[M^{\vee} / M\right]$ by

$$
e_{0}:=e_{(0,0,0)}, \quad e_{1}:=e_{\left(0,0, \rho_{k}\right)}, \quad v_{i}:=\sum_{2\langle\delta, \delta\rangle \equiv i \bmod 4} e_{\delta} .
$$

Put $q=e^{2 \pi i \tau}$. Let $\theta_{A_{1}+\delta / 2}(\tau):=\sum_{k \in \mathbb{Z}} q^{(k+\delta / 2)^{2}}(\delta \in\{0,1\})$ be the theta function of $A_{1}$-lattice. Define $f_{0}(\tau), f_{1}(\tau)$, and $\left\{c_{k, 0}(l)\right\}_{l \in \mathbb{Z}},\left\{c_{k, 1}(l)\right\}_{l \in \mathbb{Z}+1 / 4}$ by

$$
f_{0}(\tau):=\frac{\eta(2 \tau)^{8} \theta_{A_{1}}(\tau)^{k}}{\eta(\tau)^{8} \eta(4 \tau)^{8}}=\sum_{l \in \mathbb{Z}} c_{k, 0}(l) q^{l}=q^{-1}+8+2 k+O(q),
$$

$$
f_{1}(\tau):=-16 \frac{\eta(4 \tau)^{8} \theta_{A_{1}+1 / 2}(\tau)^{k}}{\eta(2 \tau)^{16}}=\sum_{l \in 1 / 4+\mathbb{Z}} 2 c_{k, 1}(l) q^{l} .
$$

Then, $f_{0}(\tau)$ is a modular form of weight $(k-8) / 2$ for $\Gamma_{0}(4)$ with the same character as that of $\theta_{A_{1}}(\tau)$. Define $g_{i}(\tau)(i \in \mathbb{Z} / 4 \mathbb{Z})$ by

$$
g_{i}(\tau)=\sum_{l \equiv i \bmod 4} c_{k, 0}(l) q^{l / 4}, \quad \sum_{i \in \mathbb{Z} / 4 \mathbb{Z}} g_{i}(\tau)=\frac{\eta(\tau / 2)^{8} \theta_{A_{1}}(\tau / 4)^{k}}{\eta(\tau)^{8} \eta(\tau / 4)^{8}}=f_{0}(\tau / 4),
$$

and a modular form of type $\rho_{M}$ by

$$
F_{k}(\tau):=f_{0}(\tau) e_{0}+f_{1}(\tau) e_{1}+\sum_{i \in \mathbb{Z} / 4 \mathbb{Z}} g_{i}(\tau) v_{i}
$$

Theorem 8.2. If $k<8$, one has $\Delta_{S_{k}}(z)^{2}=C_{k} \Psi_{\mathrm{I}_{2,10-k}(2)}\left(z, F_{k}\right)$ and the following infinite product expansion;

$$
\Delta_{S_{k}}(z)=C_{k} e^{2 \pi i\left\langle\rho_{k}, z\right\rangle} \prod_{\delta \in\{0,1\}} \prod_{r \in \Pi_{\delta}^{+}\left(\Lambda_{k}\right)}\left(1-e^{2 \pi i\langle r, z\rangle}\right)^{c_{k, \delta}(r \cdot r / 2)}
$$

where $\Pi_{\delta}^{+}\left(\Lambda_{k}\right):=\left(\delta \rho_{k}+\Lambda_{k}\right) \cap \mathrm{NE}\left(\Lambda_{k, \mathbb{R}}\right)$.

Let $M \Gamma_{0}(4)=\left\{\left(\left(\begin{array}{l}a b \\ c d\end{array}\right), \sqrt{c \tau+d}\right) \in M p_{2}(\mathbb{Z}) ; c \equiv 0 \bmod 4\right\}$ be a subgroup of $M p_{2}(\mathbb{Z})$. Put $Z:=S^{2}$. 
Lemma 8.1. There exists a character $\chi$ of $M \Gamma_{0}(4)$ such that $\rho_{M}(g) e_{0}=\chi(g)^{8-k} e_{0}$.

Proof. From (8.1), it follows that ker $\rho_{M} \backslash M \Gamma_{0}(4)=\left\{Z^{a} T^{b} ; 0 \leq a, b \leq 3\right\}$ and $\rho_{M}(g) e_{\gamma}=i^{a(8-k)} e^{b \pi i\langle\gamma, \gamma\rangle} e_{\gamma}$ if $g \in \operatorname{ker} \rho_{M} \cdot Z^{a} T^{b}$. Putting $\chi(g)=i^{a}$, we get the assertion.

For $\gamma=\left(\left(\begin{array}{l}a b \\ c d\end{array}\right), \sqrt{c \tau+d}\right) \in M p_{2}(\mathbb{Z})$, put $j(\gamma, \tau):=\sqrt{c \tau+d}$. For $g \in M p_{2}(\mathbb{Z})$, we set $\left.f\right|_{g}(\tau):=f(g \cdot \tau) j(g, \tau)^{-l}$ where $l$ is the weight of $f$. The following construction of modular forms of type $\rho_{M}$ from a scalar valued modular form of higher level, is due to Borcherds ([Bo6,7]). (It is a special case of his construction.)

Proposition 8.1 ([Bo6,7]). Let $\phi(\tau)$ be a modular form for $\Gamma_{0}(4)$ with character $\chi^{8-k}$. Then, $\tilde{\phi}(\tau):=\left.\sum_{g \in \Gamma_{0}(4) \backslash \Gamma(1)} f\right|_{g}(\tau) \rho_{M}\left(g^{-1}\right) e_{0}$ is well defined and becomes a modular form of type $\rho_{M}$.

Definition 8.1. If the level of $M$ is 4 and $F=\tilde{\phi}$ for some modular form for $\Gamma_{0}(4)$ in Theorem 8.1, we write $\Psi_{M}(z, \phi)$ in stead of $\Psi_{M}(z, \tilde{\phi}) . \Psi_{M}(z, \phi)$ is said to be Borcherds's product attached to $M$ and $\phi$.

Lemma 8.2. $F_{k}(\tau)$ is a modular form of weight $(k-8) / 2$ of type $\rho_{M}$.

Proof. Pick up the representatives $\Gamma_{0}(4) \backslash \Gamma(1)=\left\{1, S, S T, S T^{2}, S T^{3}, V\right\}$. Here we put $V:=S^{-1} T^{2} S$. From (8.1), it follows that

$$
\rho_{M}\left(\left(S T^{l}\right)^{-1}\right) e_{0}=i^{\frac{k-8}{2}} 2^{-\frac{12-k}{2}} \sum_{j=0}^{3} i^{-l j} v_{j}, \quad \rho_{M}\left(V^{-1}\right) e_{0}=e_{1}
$$

Take $\phi(\tau)=f_{0}(\tau)$ in Proposition 8.1. As $\theta_{A_{1}}(\tau)$ is a modular form of weight $1 / 2$ with character $\chi^{-1}$ for $\Gamma_{0}(4)$ (cf. [Bo5, Theorem 4.1]), $f_{0}(\tau)$ is a modular form of weight $(k-8) / 2$ for $\Gamma_{0}(4)$ with character $\chi^{8-k}$. (Note that $\chi^{8} \equiv 1$.) Since $\left.f_{0}\right|_{S T^{l}}(\tau)=2^{(8-k) / 2} i^{-k / 2} f_{0}((\tau+l) / 4)$, comparing with (8.13), we get

$$
\left.\sum_{l=0}^{3} f_{0}\right|_{S T^{l}}(\tau) \cdot \rho_{M}\left(\left(S T^{l}\right)^{-1}\right) e_{0}=\sum_{i=0}^{3} g_{i}(\tau) v_{i}
$$

Similarly, since $\left.f_{0}\right|_{V}(\tau)=f_{1}(\tau)$, comparing with (8.13),

$$
\left.f_{0}\right|_{V}(\tau) \cdot \rho_{M}\left(V^{-1}\right) e_{0}=f_{1}(\tau) e_{1}
$$

Thus, $F_{k}(\tau)=\tilde{f}_{0}(\tau)$. As the weight of $f_{0}(\tau)$ is $(k-8) / 2$, so is $F_{k}(\tau)$.

Proof of Theorem 8.2. Since it follows from (8.9-12) that

$$
\begin{aligned}
F_{k}(\tau)= & \left(q^{-1}+8+2 k+O(q)\right) e_{0}+(8+2 k+O(q)) v_{0}+O\left(q^{1 / 4}\right) v_{1}+O\left(q^{1 / 2}\right) v_{2} \\
& +\left(q^{-1 / 4}+O\left(q^{3 / 4}\right)\right) v_{3}+O(1) e_{1}
\end{aligned}
$$

$\Psi_{T_{k}}\left(z, f_{0}\right):=\Psi_{T_{k}}\left(z, F_{k}\right)$ is a modular form for some arithmetic subgroup of $O\left(T_{k}\right)$ of weight $8+2 k$. Note that $v_{0}$ contains $e_{0}$ with multiplicity one. By Theorem 8.1 (2), the zero of $\Psi_{T_{k}}\left(z, f_{0}\right)$ consists of all the hyperplanes perpendicular to the root of $T_{k}$ with multiplicity 2 . Thus, $\Delta_{S_{k}}(z)^{2}$ and $\Psi_{T_{k}}\left(z, f_{0}\right)$ have the same zero and weight which prove the first assertion. By [Bo5, Theorem 10.4], the Weyl vector of 
$\Psi\left(z, f_{0}\right)$ is $2 \rho_{k}$. Let $f_{0}(\tau / 4)=\sum_{l \in 4^{-1} \mathbb{Z}} c(l) q^{l}$ be the Fourier expansion at the cusp. Using $c(\langle r, r\rangle / 2)=c_{0, k}(\langle 2 r, 2 r\rangle / 2)$ for any $r \in \Lambda_{k}^{\vee}$, it follows from Theorem 8.1 (3) that

$$
\begin{aligned}
\Psi_{T_{k}}\left(z, f_{0}\right)= & e^{4 \pi i\left\langle\rho_{k}, z\right\rangle} \prod_{r \in \Lambda_{k} \cap \mathrm{NE}\left(\Lambda_{k, \mathbb{R}}\right)}\left(1-e^{2 \pi i\langle r, z\rangle}\right)^{c_{0, k}\left(r^{2} / 2\right)} \\
& \times \prod_{r \in\left(\rho_{k}+\Lambda_{k}\right) \cap \mathrm{NE}\left(\Lambda_{k, \mathbb{R}}\right)}\left(1-e^{2 \pi i\langle r, z\rangle}\right)^{2 c_{1, k}\left(r^{2} / 2\right)} \\
& \times \prod_{r \in \Lambda_{k}^{\vee} \cap \mathrm{NE}\left(\Lambda_{k, \mathbb{R}}\right)}\left(1-e^{2 \pi i\langle r, z\rangle}\right)^{c\left(r^{2} / 2\right)}\left(1+e^{2 \pi i\langle r, z\rangle}\right)^{c\left(r^{2} / 2\right)} \\
= & e^{4 \pi i\left\langle\rho_{k}, z\right\rangle} \prod_{r \in \Pi_{0}^{+}\left(\Lambda_{k}\right)}\left(1-e^{2 \pi i\langle r, z\rangle}\right)^{2 c_{0, k}\left(r^{2} / 2\right)} \prod_{r \in \Pi_{1}^{+}\left(\Lambda_{k}\right)}\left(1-e^{2 \pi i\langle r, z\rangle}\right)^{2 c_{1, k}\left(r^{2} / 2\right)} \\
= & {\left[e^{2 \pi i\left\langle\rho_{k}, z\right\rangle} \prod_{\delta \in\{0,1\}} \prod_{r \in \Pi_{\delta}^{+}\left(\Lambda_{k}\right)}\left(1-e^{2 \pi i\langle r, z\rangle}\right)^{c_{\delta, k}\left(r^{2} / 2\right)}\right]^{2} \cdot \square }
\end{aligned}
$$

8.3 Nikulin's $K 3$ Surfaces. For $\Lambda=\mathrm{II}_{1,1} \oplus E_{8}(-2)$, a $\Lambda$-2-elementary $K 3$ surface is one of the exceptional type $((r, l, \delta)=(10,8,0))$ in Theorem 2.5 discovered by Nikulin ([Ni4]). It is an elliptic $K 3$ surface whose fixed locus consists of two smooth fibers. Let $\Psi_{\Lambda^{\perp}}:=\left.\Phi\right|_{\Omega_{\Lambda}}$ be the same automorphic form as in Corollary 6.1.

Put $\theta_{\Lambda_{16}+\delta}(\tau)=\sum_{\lambda+\delta \in \Lambda_{16}} q^{\lambda^{2} / 2}$ for the theta series of $\Lambda_{16}$ where $\delta \in \Lambda_{16}^{\vee} / \Lambda_{16}$. By [Bo2], there exists an involution $I$ on $\Lambda_{24}$ (the Leech lattice) whose fixed lattice is $E_{8}(2)$ and anti-fixed lattice is $\Lambda_{16}$. As $\Lambda_{24}$ is self-dual, there exists a canonical identification $\Lambda_{16}^{\vee} / \Lambda_{16} \cong E_{8}(2)^{\vee} / E_{8}(2)=\Lambda^{\vee} / \Lambda$ via which we identify the generators of the standard basis of the group rings $\mathbb{C}\left[\Lambda_{16}^{\vee} / \Lambda_{16}\right]$ and that of $\mathbb{C}\left[\Lambda^{\vee} / \Lambda\right]$ (cf. $\left[\right.$ Bo5, §4]). Under this identification, $\rho_{\Lambda_{16}}=\rho_{\Lambda}$ because $\delta\left(\Lambda_{16}\right)=\delta(\Lambda)=0$. Let $\Theta_{\Lambda_{16}}(\tau)=\sum_{\delta \in \Lambda_{16}^{\vee} / \Lambda_{16}} \theta_{\Lambda_{16}+\delta}(\tau) e_{\delta}$ be the theta series of $\Lambda_{16}$. Let $\left\{c_{\delta}(k)\right\}$ be the Fourier coefficients of the following modular form;

$$
\Theta_{\Lambda_{16}}(\tau) / \Delta(\tau)=\sum_{\delta \in \Lambda_{16}^{\vee} / \Lambda_{16}} e_{\delta} \theta_{\Lambda_{16}+\delta}(\tau) / \Delta(\tau)=\sum_{\delta \in \Lambda_{16}^{\vee} / \Lambda_{16}} \sum_{k \in \mathbb{Z}} c_{\delta}(k) q^{k / 2} e_{\delta} .
$$

Theorem 8.3. $\Psi_{\Lambda^{\perp}}(z)=\Psi_{\Lambda \oplus \mathrm{II}_{1,1}}\left(z, \Theta_{\Lambda_{16}} / \Delta\right)$.

Proof. From [C-S, Chap.4] (note that $q=e^{\pi i \tau}$ in [C-S] although $q=e^{2 \pi i \tau}$ here), it follows that $\theta_{\Lambda_{16}}(\tau)=1+O\left(q^{2}\right), 1 / \Delta(\tau)=q^{-1}+24+O(q)$, and

$$
\begin{aligned}
\theta_{\Lambda_{16}}(\tau)= & \sqrt{\operatorname{det} \Lambda_{16}}(i / \tau)^{8} \theta_{\Lambda_{16}}(-1 / \tau) \\
= & 2^{4} \cdot 2^{-9}\left[\left\{\theta_{3}(\tau)^{2}-\theta_{2}(\tau)^{2}\right\}^{8}+\left\{\theta_{3}(\tau)^{2}+\theta_{2}(\tau)^{2}\right\}^{8}+\theta_{3}(\tau)^{8} \theta_{2}(\tau)^{8}\right. \\
& \left.+30\left\{\theta_{3}(\tau)^{2}-\theta_{2}(\tau)^{2}\right\}^{4} \cdot\left\{\theta_{3}(\tau)^{2}+\theta_{2}(\tau)^{2}\right\}^{4}\right] \\
= & 2^{-5}\left[\left(1-2 q^{1 / 4}\right)^{16}+\left(1+2 q^{1 / 4}\right)^{16}+\left(4 q^{1 / 4}\right)^{4}\left(1+4 q^{1 / 2}\right)^{4}\right. \\
& \left.+30\left(1-2 q^{1 / 4}\right)^{8}\left(1+2 q^{1 / 4}\right)^{8}\right]+O(q)=1+O(q) .
\end{aligned}
$$

In particular, $\delta \neq 0$ implies that $\theta_{\Lambda_{16}+\delta}(\tau)=O(q)$ and thus

$$
\Theta_{\Lambda_{16}}(\tau) / \Delta(\tau)=\left(q^{-1}+24+O(q)\right) e_{0}+\sum_{\delta \neq 0} O(1) e_{\delta}
$$


From Thereom 8.1, it follows that $\Psi_{\Lambda \oplus \mathrm{II}_{1,1}}(z, F)$ is a holomorphic modular form of weight 12 whose zero divisor coincides with the discriminant. Comparing weight and zeros, we get the assertion.

\section{§9. GKM Superalgebras Arizing from $\Delta_{M}$}

9.1 Generalized Kac-Moody Superalgebras and $K 3$ Surfaces. Following [G-N2], let us recall generalized Kac-Moody superalgebras (GKM superalgebra for short) associated to an algebraic $K 3$ surface. (For the general theory of GKM (super)algebras, see [Bo1,2] and [G-N1-3].)

Let $X$ be an algebraic $K 3$ surface and $S:=\operatorname{Pic}_{X}$ its Picard lattice. Let $\operatorname{Exc}(S)$ be the set of all -2 -curves in $X$. Let $C(S)=\left\{v \in S_{\mathbb{R}} ;\langle v, v\rangle>0\right\}$ be the light cone of $S$ and $C^{+}(S)$ be the connected component containing the ample class. Let $\operatorname{Am}\left(S_{\mathbb{R}}\right)$ be the ample cone: $\operatorname{Am}\left(S_{\mathbb{R}}\right):=\left\{l \in C^{+}(S) ;\langle l, \delta\rangle>0, \forall \delta \in \operatorname{Exc}(S)\right\}$. Put $\overline{\operatorname{Am}}(S):=\operatorname{Am}\left(S_{\mathbb{R}}\right) \cap S^{\vee}$ where closure is considered in $C^{+}(S)$. Let $W(S)$ be the Weyl group of $S$. Then, $\Delta(S)=W(S)(\operatorname{Exc}(S))$ and $\overline{\operatorname{Am}}\left(S_{\mathbb{R}}\right)$ is the fundamental domain for the action of $W(S)$ on $C^{+}(S)$.

To define a Lie superalgebra associated to $X$, we need the set of simple roots. Let us put ${ }_{s} \Delta^{r e}:=\operatorname{Exc}(S)$ for the set of all simple real roots. Let ${ }_{s} \Delta_{0}^{i m}$ (resp. $\left.{ }_{s} \Delta_{\overline{1}}^{i m}\right)$ be a sequences of elements in $\overline{\operatorname{Am}}(S)$ such that any $a \in \overline{\operatorname{Am}}(S)$ can appear in ${ }_{s} \Delta_{*}^{i m}$ finitely many times;

$$
{ }_{s} \Delta_{\bar{*}}^{i m}=\left\{m(a)_{\bar{*}} a ; a \in \overline{\operatorname{Am}}(S),\langle a, a\rangle>0\right\} \cup\left\{\tau(a)_{\bar{*}} a ; a \in \overline{\operatorname{Am}}(S),\langle a, a\rangle=0\right\} .
$$

Here $m(a)_{\bar{*}}, \tau(a)_{\bar{*}} \in \mathbb{Z}$, and $m(a)_{\bar{*}} a$ (resp. $\left.\tau(a)_{\bar{*}} a\right)$ implies that $a$ appears $m(a)_{\bar{*}}$ (resp. $\tau(a)_{\bar{*}}$ ) times in ${ }_{s} \Delta_{*}^{i m}$. An element of ${ }_{s} \Delta_{\overline{0}}^{i m}$ (resp. ${ }_{s} \Delta_{\overline{1}}^{i m}$ ) is called a simple even (resp. odd) imaginary root. Put ${ }_{s} \Delta^{i m}:={ }_{s} \Delta_{\overline{0}}^{i m} \cup_{s} \Delta_{\overline{1}}^{i m}$ for the set of all simple imaginary roots, and ${ }_{s} \Delta:={ }_{s} \Delta^{r e} \cup{ }_{s} \Delta^{i m}$ for the set of all simple roots. Let us write ${ }_{s} \Delta=\left\{h_{i}\right\}_{i \in I}\left(h_{i} \in S^{\vee}\right)$. Let $A=\left(a_{i j}\right)_{i, j \in I}$ be the Gramm matrix. Namely, $a_{i j}=\left\langle h_{i}, h_{j}\right\rangle$. As $A$ satisfies the conditions of generalized Cartan matrix ([Bo1]):

(1) $i \neq j$ implies $a_{i j} \geq 0, \quad(2) h_{i} \in{ }_{s} \Delta^{r e}$ implies $a_{i i}=-2$ and $a_{i j} \in \mathbb{Z}$, we get a GKM superalgebra $\mathfrak{g}\left(S,{ }_{s} \Delta^{i m}\right):=\mathfrak{g}^{\prime}(A)$. (See [Bo1,2], [G-N1,2] for details.) Let $\Pi^{+}:=\sum_{\alpha \epsilon_{s} \Delta} \mathbb{Z}_{+} \alpha \backslash\{0\} \subset \operatorname{NE}(S):=\operatorname{NE}\left(S_{\mathbb{R}}\right) \cap S^{\vee}$ be the set of positive roots where $\operatorname{NE}\left(S_{\mathbb{R}}\right)$ is the dual cone (Mori cone) to the ample cone $\operatorname{Am}\left(S_{\mathbb{R}}\right)$. Let $\mathfrak{g}_{\alpha}$ be the root space attached to $\alpha \in \Pi^{+} \cup\{0\} \cup-\Pi^{+}$. Then, $\mathfrak{g}\left(S,{ }_{s} \Delta^{i m}\right)$ admits root space decomposition: $\mathfrak{g}\left(S,{ }_{s} \Delta^{i m}\right)=\left(\oplus_{\alpha \in \Pi^{+}} \mathfrak{g}_{\alpha}\right) \oplus \mathfrak{g}_{0} \oplus\left(\oplus_{\alpha \in \Pi^{+}} \mathfrak{g}_{-\alpha}\right), \mathfrak{g}_{0}=S_{\mathbb{R}}$. According to the decomposition into even and odd part, we get $\mathfrak{g}_{\alpha}=\mathfrak{g}_{\alpha, \overline{0}} \oplus \mathfrak{g}_{\alpha, \overline{1}}$. Multiplicity of $\alpha \in \Pi^{+}$is defined by $\operatorname{mult}(\alpha):=\operatorname{dim} \mathfrak{g}_{\alpha, \overline{0}}-\operatorname{dim} \mathfrak{g}_{\alpha, \overline{1}} \in \mathbb{Z}$.

In view of Borcherds's works, it is the denominator formula that connects GKM superalgebras and automorphic forms. We recall it when $S$ admits a Weyl vector.

Definition 9.1. $\rho \in S_{\mathbb{Q}}$ is a Weyl vector if $\langle\rho, \delta\rangle=1$ for all $\delta \in \operatorname{Exc}(S)$.

For $a \in{ }_{s} \Delta^{i m}$, define $m(a) \in \mathbb{Z}$ by (1) $m(a)=m(a)_{\overline{0}}-m(a)_{\overline{1}}$ if $\langle a, a\rangle>0$, and (2) the following formal series if $\langle a, a\rangle=0$ and $a$ is primitive,

$$
\prod_{n=1}^{\infty}\left(1-q^{n}\right)^{\tau(n a)}=1-\sum_{k=1}^{\infty} m(k a) q^{k} \quad\left(\tau(n a):=\tau(n a)_{\overline{0}}-\tau(n a)_{\overline{1}}\right) .
$$


Theorem 9.1 ([Bo1,2] (cf. [G-N1,2])). Suppose that $S$ has a Weyl vector $\rho$. (1) For a GKM superalgebra $\mathfrak{g}\left(S,{ }_{s} \Delta^{i m}\right)$, one has the following identity;

$$
\begin{aligned}
\Phi_{\mathfrak{g}\left(S,{ }_{s} \Delta^{i m}\right)}(z): & =\sum_{w \in W(S)} \operatorname{det}(w)\left\{e^{2 \pi i\langle w(\rho), z\rangle}-\sum_{r \in_{s} \Delta^{i m}} m(r) e^{2 \pi i\langle w(\rho+r), z\rangle}\right\} \\
& =e^{2 \pi i\langle\rho, z\rangle} \prod_{\alpha \in \Pi^{+}}\left(1-e^{2 \pi i\langle\alpha, z\rangle}\right)^{\operatorname{mult}(\alpha)}
\end{aligned}
$$

The formal series $\Phi_{\mathfrak{g}\left(S,{ }_{s} \Delta^{i m}\right)}(z)$ is said to be the denominator function.

(2) Let $\Psi(y)$ be a formal series with the following integral Fourier expansion:

$$
\Psi(z)=\sum_{w \in W(S)} \operatorname{det}(w)\left\{e^{2 \pi i\langle w(\rho), z\rangle}-\sum_{r \in \overline{\operatorname{Am}}(S)} m(r) e^{2 \pi i\langle w(\rho+r), z\rangle}\right\} .
$$

Define $\tau(n r) \in \mathbb{Z}$ by (8.2) for primitive norm zero $r \in \overline{\operatorname{Am}}(S)$ and $n \in \mathbb{N}$. Let $\mathfrak{g}\left(S,{ }_{s} \Delta^{i m}\right)$ be the $G K M$ superalgebra whose simple imaginary roots are

$$
\begin{aligned}
& { }_{s} \Delta_{\overline{0}}^{i m}=\{m(r) r ; m(r)>0,\langle r, r\rangle>0\} \cup\{\tau(r) r ; \tau(r)>0,\langle r, r\rangle=0\}, \\
& { }_{s} \Delta_{\overline{1}}^{i m}=\{-m(r) r ; m(r)<0,\langle r, r\rangle>0\} \cup\{-\tau(r) r ; \tau(r)<0,\langle r, r\rangle=0\} .
\end{aligned}
$$

Then, $\Phi_{\mathfrak{g}\left(S,{ }_{s} \Delta^{i m}\right)}(z)=\Psi(z)$.

Among all the GKM superalgebras associated to $K 3$ surfaces, one of the fake monster Lie algebras constructed by Borcherds is the most beautiful and interesting.

Let $\Lambda, S$ and $T$ be the 2-elementary lattices defined by $\Lambda:=\mathrm{II}_{1,1} \oplus E_{8}(-2)$, $T=\mathrm{II}_{1,1}(2) \oplus \Lambda, S=T^{\perp}=\mathrm{II}_{1,1}(2) \oplus E_{8}(-2)$. A 2-elementary $K 3$ surface of type $S$ is the universal cover of an Enriques surface and $T$ is the transcendental lattice. The period domain $\Omega_{S}$ is realized as the tube domain $\Lambda_{\mathbb{R}}+\sqrt{-1} C(\Lambda)$ as before (cf. $(8.7))$. Set $\rho:=(0,1,0), \rho^{\prime}:=(1,0,0)$. Then, $\rho$ is a Weyl vector of $\Lambda$.

Theorem 9.2 ([Bo2,4]). There exists a GKM superalgebra $\mathfrak{g}\left(\Lambda,{ }_{s} \Delta^{i m}\right)$ (one of the fake monster Lie algebras) whose denominator function, $\Phi$, is the automorphic form over $\Omega_{S}$ of weight 4 with zero divisor $\mathcal{D}_{S}$. For $\operatorname{Im} y \gg 0$, one has the following;

$$
\begin{aligned}
\Phi(z) & =\sum_{w \in W(\Lambda)} \operatorname{det}(w) e^{2 \pi i\langle\rho, w(z)\rangle} \prod_{n>0}\left(1-e^{2 \pi i n\langle\rho, w(z)\rangle}\right)^{(-1)^{n} 8} \\
& =e^{2 \pi i\langle\rho, z\rangle} \prod_{r \in \Pi^{+}}\left(1-e^{2 \pi i\langle r, z\rangle}\right)^{(-1)^{r \cdot\left(\rho-\rho^{\prime}\right)} c\left(r^{2} / 2\right)}
\end{aligned}
$$

where $\Pi^{+}=\mathbb{N} \rho \cup\{r \in \Lambda ; \rho \cdot r>0\}$ and $\sum_{n \geq-1} c(n) q^{n}=\eta(\tau)^{-8} \eta(2 \tau)^{8} \eta(4 \tau)^{-8}$.

Theorem 9.3. There exists a constant $C_{S} \neq 0$ such that $\Delta_{S}=C_{S} \Phi . \Phi(z)^{2}$ is Borcherds's product attached to the modular form $F_{0}$ of (8.12).

Proof. Comparing the weight and zero of $\Delta_{S}$ and $\Phi$, we get the first assertion. Regarding $\mathrm{I}_{1,9}(2)$ as a sublattice of $\Lambda$, we can prove the second assertion in the same manner as Theorem 8.1.

Remark. We remark that $\mathrm{I}_{2,10}(2)$ (not the transcendental lattices $\mathrm{II}_{1,1} \oplus \mathrm{II}_{1,9}(-2)$ ) is used in (3) to construct Borcherds's product. In $[\mathrm{A}],[\mathrm{Kn}]$, Allcock and Kondō uses the similar relation between $\Lambda$ and $\mathrm{I}_{1,9}$ to study the moduli space of Enriques surfaces.

9.2 GKM Superalgebras Arising from $\boldsymbol{\Delta}_{\boldsymbol{M}}$. We keep the notations in $\S 8.1$, and study two classes of 2-elementary $K 3$ surfaces as before. 
Theorem 9.4. There exists a GKM superalgebra attached to $\Lambda_{k}$ whose denominator function is (up to a constant) $\Delta_{S_{k}}(z)$.

Proof. In view of Theorem $9.1(2)$, it is enough to show that $\Delta_{S_{k}}(z)$ admits the following integral Fourier expansion at the cusp;

$$
\Delta_{S_{k}}(z)=C_{S_{k}} \sum_{w \in W\left(\Lambda_{k}\right)} \operatorname{det}(w)\left\{e^{2 \pi i\left\langle w\left(\rho_{k}\right), z\right\rangle}-\sum_{r \in \overline{\mathrm{Am}}\left(\Lambda_{k}\right)} m(r) e^{2 \pi i\left\langle w\left(\rho_{k}+r\right), z\right\rangle}\right\}
$$

By Theorem 8.2, $\Delta_{S_{k}}(z)$ has the following Fourier expansion at the cusp;

$$
\Delta_{S_{k}}(z)=C_{S_{k}} \sum_{r \in \Lambda_{k}^{\vee} \cap C\left(\Lambda_{k}\right)^{+}} n(r) e^{2 \pi i\langle r, z\rangle}
$$

where $n(r) \in \mathbb{Z}$. By (8.4), (8.5), Theorems 7.3 and 9.2 , one has

$$
\Delta_{S_{k}}(w(z))=\lim _{v \rightarrow z}\left(\frac{i}{2 \pi}\right)^{k} \frac{\Phi(w(v))}{\prod_{i=0}^{k-1}\left\langle w(v), \delta_{i}\right\rangle}=\operatorname{det}(w) \Delta_{S_{k}}(z) \quad\left(w \in W\left(\Lambda_{k}\right)\right)
$$

because $w\left(\delta_{i}\right)=\delta_{i}$ for $i \leq k-1$. Namely, $n(w(r))=\operatorname{det}(w) n(r)$ for any $r \in \Lambda_{k}^{\vee}$ and $w \in W\left(\Lambda_{k}\right)$. Together with the same argument as [G-N1, Theorem 2.3 (a)], one has $r-\rho_{k} \in \overline{\operatorname{Am}}\left(\Lambda_{k}\right)$ if $n(r) \neq 0$. Putting $m(r):=-n\left(r+\rho_{k}\right),(9.4)$ becomes

$$
\Delta_{S_{k}}(z)=C_{S_{k}} \sum_{w \in W\left(\Lambda_{k}\right)} \operatorname{det}(w) \sum_{r \in \overline{\operatorname{Am}}\left(\Lambda_{k}\right)}-m(r) e^{2 \pi i\left\langle w\left(\rho_{k}+r\right), z\right\rangle}
$$

By Theorem 8.2, $-m(0)=1$ which, together with (9.6), yields the assertion.

To study the case of 2-elementary $K 3$ surface of type $\Lambda$, Borcherds's $\Phi$-function of rank 26 is crucial. Let $\Lambda_{24}$ be the Leech lattice, and put $L:=\mathrm{II}_{1,1} \oplus \Lambda_{24}(-1)$. Let $\rho=(0,1,0)$ be a Weyl vector of $L$.

Theorem 9.5 ([Bo2,3]). The denominator function $\Phi$ of the fake monster Lie algebra is the automorphic form over $\Omega_{\mathrm{II}_{1,1} \oplus L}$ of weight 12 with only simple zero along the discriminant. The denominator formula becomes

$$
\Phi(z)=\sum_{w \in W(L)} \sum_{n>0} \operatorname{det}(w) \tau(n) e^{2 \pi i\langle w(\rho), z\rangle}=e^{2 \pi i\langle\rho, z\rangle} \prod_{r \in \Pi^{+}}\left(1-e^{2 \pi i\langle r, z\rangle}\right)^{p_{24}\left(1-r^{2} / 2\right)}
$$

where $\tau(n)$ is the Ramanujan $\tau$-function, $p_{24}(n)$ the number of partitions of $n$ into 24 colors, and $\Pi^{+}=\mathbb{N} \rho \cup\{r \in \Lambda ; \rho \cdot r>0\}$.

Theroem 9.6. $\Psi_{\Lambda^{\perp}}$ is the denominator function of a GKM superalgebra.

Proof. By Theorem 8.3, $\Psi_{\Lambda^{\perp}}(z)$ has the similar Fourier expansion as (9.4). Since $\Psi_{\Lambda^{\perp}}=\left.\Phi\right|_{\Omega_{\mathrm{II}_{1,1} \oplus \Lambda}}$, it follows from Theorem 9.5 that $\Psi_{\Lambda^{\perp}}(w(z))=\operatorname{det}(w) \Psi_{\Lambda^{\perp}}(z)$. Thus, we get the assertion in the same manner as Theorem 9.4. 


\section{$\S 10$. An Explicit Formula for $\Delta_{A_{1}}$ and $\Delta_{\mathrm{II}_{1,1}(2)}$}

We determine an explicit formula for $\Delta_{A_{1}}$ and $\Delta_{\mathrm{II}_{1,1}(2)}$ in this section.

10.1 An Explicit Formula for $\boldsymbol{\Delta}_{\boldsymbol{A}_{\mathbf{1}}}$. Let $(X, \iota)$ be a 2-elementary $K 3$ surface of type $A_{1}$. Then, $X / \iota=\mathbb{P}^{2}$ and the fixed curve $X^{\iota}$ is a smooth plane sextic curve where the quotient map $X \rightarrow \mathbb{P}^{2}$ is the morphism associated to the complete linear system of ample line bundle of degree 2. As $X^{\iota} \subset \mathbb{P}^{2}$ has an ambiguity of $P G L(3, \mathbb{C})$ (arizing from a choice of 3 sections), there exists a morphism $i: \mathcal{M}_{A_{1}}^{0} \ni$ $[(X, \iota)] \rightarrow\left[X^{\iota}\right] \in H_{6}^{s m} / P G L(3, \mathbb{C})$ where $H_{6}^{s m}=\mathbb{P}\left(\operatorname{Sym}^{6} \mathbb{C}^{3}\right)^{\vee} \backslash D_{6}$ is the set of all smooth sextic curves in $\mathbb{P}^{2}$, and $D_{6}$ is the discriminant locus of universal plane sextic curves $\pi: \mathcal{C}_{6} \rightarrow H_{6}=\mathbb{P}\left(\operatorname{Sym}^{6} \mathbb{C}^{3}\right)^{\vee}$. Note that $\xi=\left(\xi_{I}\right) \in \mathbb{P}\left(\operatorname{Sym}^{6} \mathbb{C}^{3}\right)^{\vee}$ corresponds to the curve $C_{\xi}=\left\{x \in \mathbb{P}^{2} ; \sum_{|I|=6} \xi_{I} x^{I}=0\right\}$. Conversely, if $\xi, \xi^{\prime} \in H_{6}^{s m}$ are in the same orbit of $P G L(3, \mathbb{C}), C_{\xi}$ and $C_{\xi^{\prime}}$ are projectively equivalent and thus the double covers of $\mathbb{P}^{2}$ blanching along $C_{\xi}$ and $C_{\xi^{\prime}}$ are isomorphic. In this way, one verify $i: \mathcal{M}_{A_{1}}^{0} \cong H_{6}^{s m} / P G L(3, \mathbb{C})$ ([Sha $\left.]\right)$.

We denote by $\lambda\left(\mathcal{C}_{6} / H_{6}^{s m}\right)$ the determinant of cohomology in the sense of $\S 3.1$. Let $\mathrm{Jac}: H_{6}^{s m} \ni \xi \rightarrow\left[\operatorname{Jac}\left(C_{\xi}\right)\right] \in \mathcal{A}_{10}$ be the Torelli map. As $j_{A_{1}}=$ Jac $\circ i$ and $(\mathrm{Jac})^{*} \mathcal{F}_{10}=\lambda\left(\mathcal{C}_{6} / H_{6}^{s m}\right)^{G L(3, \mathbb{C})}$, we find $\lambda_{A_{1}}=i^{*} \lambda\left(\mathcal{C}_{6} / H_{6}^{s m}\right)^{G L(3, \mathbb{C})}$ where $\lambda\left(\mathcal{C}_{6} / H_{6}^{s m}\right)^{G L(3, \mathbb{C})}$ is the sheaf of $G L(3, \mathbb{C})$-invariant sections of $\lambda\left(\mathcal{C}_{6} / H_{6}^{s m}\right)$. Moreover, this identification is an isometry if $\lambda_{A_{1}}$ is equipped with the Petersson metric and if $i^{*} \lambda\left(\mathcal{C}_{6} / H_{6}^{s m}\right)^{G L(3, \mathbb{C})}$ with the $L^{2}$-metric. Our first task is to construct a section of $\lambda_{A_{1}}^{\otimes 15}$ arizing from the discriminant of plane sextics. In the sequel, we put $F(x ; \xi)=\sum_{|I|=6} \xi_{I} x^{I}$. By the Poincaré residue sequence, we get the following.

Lemma 10.1. If we denote by $x, y, z$ the homogeneous coordinates of $\mathbb{P}^{2}$, then

$$
H^{0}\left(C_{\xi}, \Omega_{C_{\xi}}^{1}\right)=\bigoplus_{i+j+k=3} \mathbb{C}_{\operatorname{Res}_{C_{\xi}}} \frac{x^{i} y^{j} z^{k}(x d y \wedge d z-y d x \wedge d z+z d x \wedge d y)}{F(x, y, z ; \xi)}
$$

We define a local section of $\lambda\left(\mathcal{C}_{6} / H_{6}^{s m}\right)$ by

$$
\omega(\xi):=\bigwedge_{i+j+k=3} \operatorname{Res}_{C_{\xi}} \frac{x^{i} y^{j} z^{k}(x d y \wedge d z-y d x \wedge d z+z d x \wedge d y)}{F(x, y, z ; \xi)} \in \operatorname{det} H^{0}\left(C_{\xi}, \Omega^{1}\right) .
$$

To be precise, if $p:\left(\operatorname{Sym}^{6} \mathbb{C}^{3}\right)^{\vee} \backslash\{0\} \rightarrow \mathbb{P}\left(\operatorname{Sym}^{6} \mathbb{C}^{3}\right)^{\vee}$ is the natural projection, then $\omega$ is a section of $p^{*} \lambda\left(\mathcal{C}_{6} / H_{6}^{s m}\right)$ over $\left(\operatorname{Sym}^{6} \mathbb{C}^{3}\right)^{\vee}$.

Lemma 10.2. For any $g \in G L(3, \mathbb{C}), g^{*} \omega=\operatorname{det}(g)^{20} \omega$ where $G L(3, \mathbb{C})$ acts on $\left(\mathrm{Sym}^{6} \mathbb{C}^{3}\right)^{\vee}$ via the induced representation.

Proof. From the definition of representation of $G L(3, \mathbb{C})$ on $\left(\operatorname{Sym}^{6} \mathbb{C}^{3}\right)^{\vee}$, it follows that $F(g \cdot(x, y, z) ; g \cdot \xi)=F(x, y, z ; \xi)$. Since $G L(3, \mathbb{C})$ acts on $\operatorname{det} \operatorname{Sym}^{3} \mathbb{C}^{3}$ by $\operatorname{det}(\cdot)^{10}$, we get $g \cdot \wedge_{i+j+k=3} x^{i} y^{j} z^{k}=\operatorname{det}(g)^{10} \wedge_{i+j+k=3} x^{i} y^{j} z^{k}$. By computation, we get $g^{*}(x d y \wedge d z-y d x \wedge d z+z d x \wedge d z)=\operatorname{det}(g)(x d y \wedge d z-y d x \wedge d z+z d x \wedge d z)$. Together with all of these, we get the assertion: $g^{*}(\omega(g \cdot \xi))=\operatorname{det}(g)^{20} \omega(\xi)$.

Lemma 10.3. There exists a homogeneous polynomial $D_{6}(\xi)$ of degree 75 in the $\xi$ variable such that the discriminant locus of the plane sextics is $\operatorname{div}\left(D_{6}\right)$. Moreover, for any $g \in G L(3, \mathbb{C}), D_{6}(g \cdot \xi)=\operatorname{det}(g)^{-150} D_{6}(\xi)$. 
Proof. Let $v_{6}: \mathbb{P}\left(\mathbb{C}^{3}\right) \hookrightarrow \mathbb{P}\left(\operatorname{Sym}^{6} \mathbb{C}^{3}\right)$ be the Veronese embedding. Put $X:=$ $v_{6}\left(\mathbb{P}\left(\mathbb{C}^{3}\right)\right)$ and $X^{\vee} \subset \mathbb{P}\left(\operatorname{Sym}^{6} \mathbb{C}^{3}\right)^{\vee}$ for the projective dual variety of $\left(X, \mathcal{O}_{\mathbb{P}\left(\operatorname{Sym}^{6} \mathbb{C}^{3}\right)}(1)\right)$. By Katz's formula ([Kt, Cor. 5.6]), $X^{\vee}$ is a projective hypersurface of degree

$$
\operatorname{deg} X^{\vee}=\int_{\mathbb{P}\left(\mathbb{C}^{3}\right)} \frac{c\left(\mathbb{P}\left(\mathbb{C}^{3}\right)\right)}{\left(1+v_{6}^{*} c_{1}\left(\mathbb{P}\left(\mathrm{Sym}^{6} \mathbb{C}^{3}\right)\right)\right)^{2}}=\int_{\mathbb{P}\left(\mathbb{C}^{3}\right)} \frac{1+3 H+3 H^{2}}{(1+6 H)^{2}}=75
$$

where $H=c_{1}\left(\mathbb{P}\left(\mathbb{C}^{3}\right)\right)$ is the hyperplane section of $\mathbb{P}\left(\mathbb{C}^{3}\right)$. Take $D_{6}(\xi)$ as a defining equation of $X^{\vee}$. As $X^{\vee}$ is invariant under the action of $G L(3, \mathbb{C})$, there exists $l \in \mathbb{Z}$ such that $D_{6}(g \cdot \xi)=\operatorname{det}(g)^{l} D_{6}(\xi)$. Putting $g=\lambda I_{3}\left(\lambda \in \mathbb{C}^{\times}\right)$, we find $l=-150$.

Proposition 10.1. $\Delta_{6}^{2}(\xi):=D_{6}(\xi)^{2} \cdot \omega(\xi)^{\otimes 15}$ is a $G L(3, \mathbb{C})$-invariant section of $p^{*} \lambda\left(\mathcal{C}_{6} / H_{6}^{s m}\right)$. In particular, we may regard $\Delta_{6}^{2} \in H^{0}\left(\mathcal{M}_{A_{1}}^{0}, \lambda_{A_{1}}^{\otimes 15}\right)$.

Proof. The first assertion follows from Lemmas 10.2 and 10.3, and the second from $H^{0}\left(H_{6}^{s m}, \lambda\left(\mathcal{C}_{6} / H_{6}^{s m}\right)^{\otimes 15}\right)^{G L(3, \mathbb{C})}=H^{0}\left(\mathcal{M}_{A_{1}}^{0}, \lambda_{A_{1}}^{\otimes 15}\right)$.

Let $\delta_{1}, \delta_{2} \in \Delta\left(\mathrm{II}_{2,18} \oplus A_{1}(-1)\right)$ such that $\delta_{1} / 2 \in\left(\mathrm{II}_{2,18} \oplus A_{1}(-1)\right)^{\vee}$ and $\delta_{2} / 2 \notin$ $\left(\mathrm{II}_{2,18} \oplus A_{1}(-1)\right)^{\vee}$. Since $\Gamma_{A_{1}}=O\left(\mathrm{II}_{2,18} \oplus A_{1}(-1)\right)$, it follows from a theorem of Nikulin ([Ni1, Proposition 1.15.1]) that $\Delta\left(\mathrm{II}_{2,18} \oplus A_{1}(-1)\right) / \Gamma_{A_{1}}=\left\{\delta_{1}, \delta_{2}\right\}$. In particular, $\mathcal{D}_{A_{1}} / \Gamma_{A_{1}}$ consists of 2 irreducible components: $\mathcal{D}_{A_{1}} / \Gamma_{A_{1}}=H_{\delta_{1}}+H_{\delta_{2}}$.

Lemma 10.4. $\Delta_{6}^{2}$ vanishes of order 2 along $H_{\delta_{2}}$.

Proof. Since $\left\langle A_{1} \oplus \delta_{2}\right\rangle=A_{1} \oplus A_{1}(-1)$, 2-elementary $K 3$ surfaces over $H_{\delta_{2}}$ are those of type $A_{1} \oplus A_{1}(-1)$. Take a generic 2-elementary K3 surface $(X, \iota)$ of this type. Then, $\operatorname{Pic}(X / \iota)=\mathrm{I}_{1,1}$ and $X / \iota$ is a blow-up of $\mathbb{P}^{2}$ at one point. This implies that the complete linear system associated to the nef and big line bundle of degree 2 maps $X$ to $\mathbb{P}^{2}$ and $X^{\iota}$ to a nodal sextic curve with one singular point. Conversely, given such a nodal sextic curve, the minimal resolution of the double cover of $\mathbb{P}^{2}$ blanching along it is a 2-elementary $K 3$ surface of type $A_{1} \oplus A_{1}(-1)$. This extends the isomorphism $i: \mathcal{M}_{A_{1}}^{0} \cup H_{\delta_{2}}^{0} \rightarrow H_{6}^{\text {nod }} / P G L(3, \mathbb{C})$ and $\lambda_{A_{1}}=$ $i^{*} \lambda\left(\mathcal{C}_{6} / H_{6}^{\text {nod }}\right)^{G L(3, \mathbb{C})}$ where $H_{6}^{\text {nod }}$ is the set of all plane sextic curves with at most one node. By Proposition 10.1, we find $\Delta_{6}^{2} \in H^{0}\left(H_{6}^{\text {nod }}, \lambda\left(\mathcal{C}_{6} / H_{6}^{\text {nod }}\right)^{\otimes 15}\right)^{G L(3, \mathbb{C})}=$ $H^{0}\left(\mathcal{M}_{A_{1}}^{0} \cup H_{\delta_{2}}^{0}, \lambda_{A_{1}}^{\otimes 15}\right)$. To compute the vanishing order of $\Delta_{6}^{2}$, take a generic point $p \in H_{\delta_{2}}$ and its small neighborhood $U$. As $\omega$ is regarded to be a nowhere vanishing section of $\lambda_{A_{1}}$ on $U$, the assertion follows from Proposition 10.1 because $H_{\delta_{2}}=\operatorname{div}\left(D_{6}\right)$.

Lemma 10.5. $\Delta_{6}^{2}$ has an algebraic singularity along $H_{\delta_{1}}$.

Proof. By Shah's result ([Sha]), $H_{\delta_{1}}$ contracts to a point in $H_{6}^{s s} / P G L(3, \mathbb{C})$ corresponding to a smooth conic of multiplicity 3 where $H_{6}^{s s}$ is the set of all semi-stable sextic curves. If $\left\{C_{\xi_{t}}\right\}_{|t|<1}$ is a degenerating family of sextic curves such that $C_{\xi_{0}}$ is a smooth triple conic, then $\left\|\Delta_{6}^{2}\left(\xi_{t}\right)\right\|_{L^{2}} \sim|t|^{a}(\log |t|)^{b}(t \rightarrow 0)$ with some $a, b \in \mathbb{R}$. If $(U, s)$ is a holomorphic disc which meets transversally to $H_{\delta_{1}}$ at $s=0$, then

$$
\left\|i^{*} \Delta_{6}^{2}(s)\right\| \sim|s|^{l}(\log |s|)^{m} \quad(s \rightarrow 0)
$$

with some $l, m \in \mathbb{R}$ because $s$ and $t$ are algebraically related. Since $\left\langle A_{1}, \delta_{1}\right\rangle=\mathrm{II}_{1,1}$ and $g\left(\mathrm{II}_{1,1}\right)=10=g\left(A_{1}\right)$, there exists a section, $\sigma$, of $\lambda_{A_{1}}^{\otimes 15}$ over $U$ such that 
$\|\sigma(s)\| \sim 1(s \rightarrow 0)$ because $j_{A_{1}}(U)$ is away from the boundary component of the Siegel modular variety $\mathcal{A}_{10}$. Since $i^{*} \Delta_{6}^{2}(s) / \sigma(s)$ is a holomorphic function on $U \backslash\{0\}$, it must be meromorphic on $U$ because of (10.3). In particular, $m=0$ and $\Delta_{6}^{2}$ has zero (or pole) of order $l \in \mathbb{Z}$ along $H_{\delta_{1}}$.

Let $E_{1}, E_{2}$ be divisors on $\Omega_{A_{1}}$ defined by $E_{1}=\sum_{\delta \sim \delta_{1}} H_{\delta}$ and $E_{2}=\sum_{\delta \sim \delta_{2}} H_{\delta}$ where $\delta$ runs over $\Delta\left(\mathrm{II}_{2,18} \oplus A_{1}(-1)\right)$ and $\delta \sim \delta^{\prime}$ if $\delta=\gamma \cdot \delta^{\prime}$ for some $\gamma \in$ $\Gamma_{A_{1}}$. Identify $\Delta_{6}^{2}$ to be a $\Gamma_{A_{1}}$-invariant section of $\lambda_{A_{1}}^{\otimes 15}$ over $\Omega_{A_{1}}$, namely $\Delta_{6}^{2} \in$ $H^{0}\left(\Omega_{A_{1}}, \lambda_{A_{1}}^{\otimes 15}\right)^{\Gamma_{A_{1}}}$. Since the quotient map $\Omega_{A_{1}} \rightarrow \mathcal{M}_{A_{1}}$ blanches of order 2 along $\mathcal{D}_{A_{1}}=E_{1}+E_{2}$, we get the following from Lemmas 10.4 and 10.5.

Lemma 10.6. $\Delta_{6}^{2}$ vanishes of order $2 l(l \in \mathbb{Z})$ along $E_{1}$ and of order 4 along $E_{2}$.

Proposition 10.2. $\Psi_{\mathrm{II}_{2,18} \oplus A_{1}(-1)}\left(z, \Theta_{E_{7}} / \Delta\right)$ is a modular form of weight 75 with zero divisor $57 E_{1}+E_{2}$ where $\Theta_{E_{7}}(\tau)$ is the theta series of the $E_{7}$-lattice.

Proof. Since $\Theta_{E_{7}}(\tau) / \Delta(\tau)$ is a modular form of type $\rho_{\mathrm{II}_{2,18} \oplus A_{1}(-1)}$ of weight $-17 / 2$, we can construct Borcherds's product $\Psi_{\mathrm{II}_{2,18} \oplus A_{1}(-1)}\left(z, \Theta_{E_{7}} / \Delta\right)$ by Theorem 8.1. Since $\theta_{E_{7}}(\tau)=1+126 q+O\left(q^{2}\right), \theta_{E_{7}+1 / 2}(\tau)=56 q^{3 / 4}+576 q^{7 / 4}+O\left(q^{2}\right)$ and $1 / \Delta(\tau)=q^{-1}+24+O(q)$ (cf. [C-S]), we get

$$
\Theta_{E_{7}}(\tau) / \Delta(\tau)=\left(q^{-1}+150+O(q)\right) e_{0}+\left(56 q^{-1 / 4}+O\left(q^{3 / 4}\right)\right) e_{1}
$$

By Theorem 8.1, $\Psi_{\mathrm{II}_{2,18} \oplus A_{1}(-1)}\left(z, \Theta_{E_{7}} / \Delta\right)$ has weight $c_{0}(0) / 2=75$ whose zero divisor is $\sum_{\delta \in \Delta\left(\mathrm{II}_{2,18} \oplus A_{1}(-1)\right)} H_{\delta}+56 \sum_{\delta \in \Delta\left(\mathrm{II}_{2,18} \oplus A_{1}(-1)\right), \delta / 2 \in\left(\mathrm{II}_{2,18} \oplus A_{1}(-1)\right) \vee} H_{\delta}=$ $57 E_{1}+E_{2}$.

Theorem 10.1. $\Delta_{A_{1}}^{15}(z)=C_{A_{1}} \Delta_{6}^{8}(z) / \Psi_{\mathrm{II}_{2,18} \oplus A_{1}(-1)}\left(z, \Theta_{E_{7}} / \Delta\right)$.

Proof. Put $F(z):=\Delta_{6}^{8}(z) /\left[\Delta_{A_{1}}(z)^{15} \cdot \Psi_{\mathrm{II}_{2,18} \oplus A_{1}(-1)}\left(z, \Theta_{E_{7}} / \Delta\right)\right]$. Since $\Delta_{A_{1}}$ is an automorphic form of weight $(-5,4), \Delta_{6}^{2}$ of $(0,15)$ and $\Psi_{\mathrm{II}_{2,18} \oplus A_{1}(-1)}\left(z, \Theta_{E_{7}} / \Delta\right)$ of $(75,0)$ by Theorem 7.2, Propositions 10.1 and $10.2, F$ is an automorphic form of weight $(0,0)$. Namely, taking a higher power of $F$ if necessary, $F$ is a $\Gamma_{A_{1}}$-invariant meromorphic function on $\Omega_{A_{1}}$, thus a meromorphic function on the modular variety $\mathcal{M}_{A_{1}}$. From Theorem 7.2, Lemma 10.6 and Proposition 10.2, it follows that $\operatorname{div}(F)=8(l-9) E_{1}$. Since $F$ extends to a meromorphic function on the SatakeBaily-Borel compactification of $\mathcal{M}_{A_{1}}$, the residue theorem implies $l=9$ and $F$ must be a (nonzero) constant.

Remark. It seems that it is in [B-K-P-S-B] that $\Psi_{\mathrm{II}_{2,18} \oplus A_{1}(-1)}\left(z, \Theta_{E_{7}} / \Delta\right)$ first appeared. From their construction, its Weyl vector is the projection of that of $\mathrm{II}_{1,1} \oplus E_{8}(-1)^{\oplus 3}$ to $\mathrm{II}_{1,1} \oplus E_{8}(-1)^{\oplus 2} \oplus A_{1}(-1)$. They used this modular form to show that the moduli space of $K 3$ surfaces of degree 2 is quasi-affine. See [B-KP-S-B, Theorem 1.3 and Example 2.1] for the details.

10.2 An Explicit Formula for $\Delta_{\mathbf{I I}_{\mathbf{1}, \mathbf{1}}(\mathbf{2})}$. Let $(X, \iota)$ be a 2-elementary $K 3$ surface of type $\mathrm{II}_{1,1}(2)$. $(X, \iota)$ is said to be generic if $E \cdot \iota(E)>0$ for any -2-curve $E$. (Equivalently, $E+\iota(E)$ lies in the positive cone: $(E+\iota(E))^{2} \geq 0$.) Let $e, f$ be a basis of $\mathrm{II}_{1,1}(2)$ such that $e^{2}=f^{2}=0$ and $e \cdot f=2$. Let $\phi$ be a marking of $(X, \iota)$. If it is generic, we may suppose that $C_{e}:=\phi^{-1}(e)$ and $C_{f}:=\phi^{-1}(f)$ are nef divisors because $2 C_{e} \cdot E=C_{e} \cdot(E+\iota(E)) \geq 0$ for any -2-curve $E$. Thus, $X$ has two elliptic fibrations associated to the linear systems $\left|C_{e}\right|$ and $\left|C_{f}\right|$. Since $C_{e}+C_{f}$ 
is ample, the linear system $\left|C_{e}+C_{f}\right|$ induces a finite surjective morphism $\Phi: X \rightarrow$ $\mathbb{P}^{1} \times \mathbb{P}^{1}\left(\subset \mathbb{P}^{3}\right)$, because $H^{0}\left(X, \phi^{-1}(e+f)\right)=H^{0}\left(X, \phi^{-1}(e)\right) \otimes H^{0}\left(X, \phi^{-1}(f)\right)$. By the Lefschetz formula, these spaces consist of $\iota$-invariant sections. Thus $\Phi$ induces a map; $\Phi / \iota: X / \iota \rightarrow \mathbb{P}^{1} \times \mathbb{P}^{1}$. As it induces an isomorphism of the Picard lattice, it must be an isomorphism. Thus, any generic 2-elementary $K 3$ surfaces of type $\mathrm{II}_{1,1}(2)$ is realized as a double cover of $\mathbb{P}^{1} \times \mathbb{P}^{1}$ blanching along a smooth curve of bidegree $(4,4)$. By this description, we can construct the discriminant of fixed curves as that of curves of bidegree $(4,4)$ on $\mathbb{P}^{1} \times \mathbb{P}^{1}$.

Let $H_{4,4}=\mathbb{P}\left(\operatorname{Sym}^{4} \mathbb{C}^{2} \otimes \operatorname{Sym}^{4} \mathbb{C}^{2}\right)^{\vee}$ be the set of all curves of bidegree $(4,4)$ on $\mathbb{P}^{1} \times \mathbb{P}^{1}$. As before, $\xi=\left(\xi_{i j, k l}\right)_{i+j=4, k+l=4} \in H_{4,4}$ represents the curve defined by $C_{\xi}:=\left\{((x, y),(z, w)) \in \mathbb{P}^{1} \times \mathbb{P}^{1} ; \sum \xi_{i j, k l} x^{i} y^{j} z^{k} w^{l}=0\right\}$. Let $\pi: \mathcal{C}_{4,4} \rightarrow H_{4,4}$ be the universal family i.e. $\pi^{-1}(\xi)=C_{x i}$, on which acts the group of projective transformations $P G:=P(G L(2, \mathbb{C}) \times G L(2, \mathbb{C}))$. Then, above description implies an isomorphism $i: \mathcal{M}_{\mathrm{II}_{1,1}(2)}^{00} \ni[(X, \iota)] \rightarrow\left[X^{\iota}\right] \in H_{4,4}^{s m} / P G$ where $H_{4,4}^{s m}$ is the set of all smooth curves in $H_{4,4}$ and $\mathcal{M}_{\mathrm{II}_{1,1}(2)}^{00}$ is the set of all isomorphism classes of generic 2-elementary $K 3$ surfaces of type $\mathrm{II}_{1,1}(2)$. Let $\lambda\left(\mathcal{C}_{4,4} / H_{4,4}\right)$ be the determinant of cohomology on which acts $G:=G L(2, \mathbb{C}) \times G L(2, \mathbb{C})$. Note that the action of $G$ is not effective. As before, we get an identification $\lambda_{\mathrm{II}_{1,1}(2)}=i^{*} \lambda\left(\mathcal{C}_{4,4} / H_{4,4}^{\text {nod }}\right)^{G}$ on $\mathcal{M}_{\mathrm{II}_{1,1}(2)}^{00} \cup \mathcal{D}_{\mathrm{II}_{1,1}(2)}^{0}$ where $H_{4,4}^{\text {nod }}$ is the set of all curve of bidegree $(4,4)$ with at most one node. Put $F(x, y, z, w ; \xi):=\sum_{i+j=k+l=4} \xi_{i j, k l} x^{i} y^{j} z^{k} w^{l}$. We regard $(x, y)$ and $(z, w)$ as the homogeneous coordinates of $\mathbb{P}^{1}$. By the Poincaré residue sequence, we get the following.

\section{Lemma 10.7.}

$$
H^{0}\left(C_{\xi}, \Omega_{C_{\xi}}^{1}\right)=\bigoplus_{i+j=k+l=2} \mathbb{C} \operatorname{Res}_{C_{\xi}} \frac{x^{i} y^{j} z^{k} w^{l}(x d y-y d x) \wedge(z d w-w d z)}{F(x, y, z, w ; \xi)} .
$$

As before, let us define a local section of $\lambda\left(\mathcal{C}_{4,4} / H_{4,4}^{\text {nod }}\right)$ by

$$
\omega(\xi):=\bigwedge_{i+j=k+l=2} \operatorname{Res}_{C_{\xi}} \frac{x^{i} y^{j} z^{k} w^{l}(x d y-y d x) \wedge(z d w-w d z)}{F(x, y, z, w ; \xi)} \in \operatorname{det} H^{0}\left(C_{\xi}, \Omega^{1}\right) .
$$

Lemma 10.8. For any $(g, h) \in G,(g, h)^{*} \omega=(\operatorname{det}(g) \operatorname{det}(h))^{18} \omega$.

Proof. Since the proof is similar to that of Lemma 10.2, we leave it to the reader.

Lemma 10.9. There exists a homogeneous polynomial $D_{4,4}(\xi)$ of degree 68 in the $\xi$-variable such that the discriminant locus of $\pi: \mathcal{C}_{4,4} \rightarrow H_{4,4}$ coincides with $\operatorname{div}\left(D_{4,4}\right)$. Moreover, for any $(g, h) \in G, D_{4,4}((g, h) \cdot \xi)=(\operatorname{det}(g) \operatorname{det}(h))^{-136} D_{4,4}(\xi)$.

Proof. Let $Q=\mathbb{P}^{1} \times \mathbb{P}^{1}\left(\subset \mathbb{P}^{3}\right)$ be a hyperquadric. Since the line bundle of bidegree $(4,4)$ is $-2 K_{Q}$ ( $K_{Q}$ is the canonical bundle), let us consider the Veronese embedding $v_{4,4}: Q \hookrightarrow \mathbb{P}\left(H^{0}\left(Q,-2 K_{Q}\right)\right)^{\vee}$. Let $X:=v_{4,4}(Q)$ be the image and $X^{\vee}$ be the

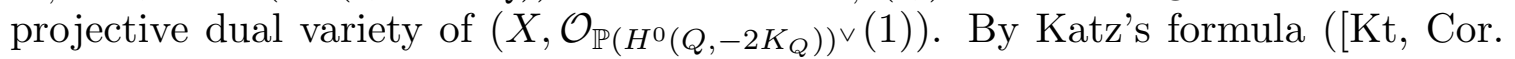
$5.6]), X^{\vee}$ is a projective hypersurface of degree

$$
\operatorname{deg} X^{\vee}=\int_{Q} \frac{c(Q)}{\left(1+v_{4,4}^{*} c_{1}\left(\mathbb{P}\left(H^{0}\left(Q,-2 K_{Q}\right)\right)^{\vee}\right)\right)^{2}}=\int_{Q} \frac{1+2 H+2 H^{2}}{(1+4 H)^{2}}=68
$$


where $H=\mathcal{O}_{\mathbb{P}^{3}}(1)$. Let $D_{4,4}(\xi)$ be a defining equation of $X^{\vee}$. Since $X^{\vee}$ is stable under the action of $G$, there exists $l \in \mathbb{Z}$ such that $D_{4,4}((g, h) \cdot \xi)=$ $(\operatorname{det}(g) \operatorname{det}(h))^{l} D_{4,4}(\xi)$. Putting $g=\lambda I_{2}, h=I_{2}$, we find $l=-136$.

Proposition 10.3. $\Delta_{4,4}^{9}(\xi):=D_{4,4}(\xi)^{9} \cdot \omega(\xi)^{\otimes 68}$ becomes a $G$-invariant section of $\lambda\left(\mathcal{C}_{4,4} / H_{4,4}^{\text {nod }}\right)$. In particular, we may regard $\Delta_{4,4}(\xi)^{9} \in H^{0}\left(\mathcal{M}_{\mathrm{II}_{1,1}(2)}^{00}, \lambda_{\mathrm{II}_{1,1}(2)}^{\otimes 68}\right)$.

Proof. The assertion follows from Lemmas 10.8 and 10.9.

Lemma 10.10. The discriminant locus $H:=\mathcal{D}_{\mathrm{II}_{1,1}(2)} / \Gamma_{\mathrm{II}_{1,1}(2)}$ is an irreducible divisor of $\mathcal{M}_{\mathrm{II}_{1,1}(2)}$. $\Delta_{4,4}^{9}$ vanishes of order 9 along $H$.

Proof. The first assertion follows from Nikulin's theory ([Ni, Proposition 1.15.1]), and the second from Proposition 10.3.

Let us discuss special 2-elementary $K 3$ surfaces of type $\mathrm{II}_{1,1}(2)$. A 2-elementary $K 3$ surface of type $\mathrm{II}_{1,1}(2),(X, \iota)$, is said to be special if it has a -2-curve $E$ such that $E$ and $\iota(E)$ is disjoint. (This definition is analogous to that of Enriques surfaces ([B-P-V-V, Theorem 18.2], [Na, Remark 4.6]).) Let $\phi$ be a marking of $(X, \iota)$ and $I:=\phi \circ \iota^{*} \phi^{-1}$ be the involution induced by $\iota$. Put $\delta:=\phi(E) \in \Delta\left(L_{K 3}\right)$. Then, $\langle\delta, I(\delta)\rangle=0$ by assumption. Let $T:=\left(\mathrm{II}_{1,1}(2)\right)^{\perp}=\mathrm{II}_{1,1}(2) \oplus \mathrm{I}_{1,17}$ be the transcendental lattice. Let $p: L_{K 3} \rightarrow T^{\vee}$ be the orthogonal projection. We set $d:=p(\delta)=(\delta-I(\delta)) / 2 \in T^{\vee}$. Then, $d^{2}=-1$ and $d \equiv(e+f) / 2 \bmod T$ where $\{e, f\}$ is the basis of $\mathrm{II}_{1,1}(2)$ as before. Conversely, if $d^{\prime} \in T^{\vee}$ is a vector such that $\left(d^{\prime}\right)^{2}=-1$ and $d^{\prime} \equiv(e+f) / 2 \bmod T$, one can easily verify that there exists $\delta^{\prime} \in \Delta\left(L_{K 3}\right)$ such that $\left\langle\delta^{\prime}, I\left(\delta^{\prime}\right)\right\rangle=0$ and $d^{\prime}=p\left(\delta^{\prime}\right)$.

Lemma 10.11. $(X, \iota)$ is a special 2-elementary $K 3$ surface of type $\mathrm{II}_{1,1}(2)$ iff its period lies on the divisor $\mathcal{D}_{\mathrm{II}_{1,1}(2)}^{\prime}:=\sum_{d \in T^{\vee}, d^{2}=-1} H_{d}$.

Proof. Suppose that $(X, \iota)$ is special. Let $\omega$ be a canonical form of $(X, \iota)$. Let $E$ be a -2 -curve as above. Since $\langle\omega, E\rangle=\langle\phi(\omega), d\rangle=0$, we get $\phi(\omega) \in H_{d}$. Conversely, let $(X, \iota, \phi)$ be a marked 2-elementary $K 3$ surface of type $\mathrm{II}_{1,1}(2)$ whose period lies on $H_{d} \subset \mathcal{D}^{\prime}$. Let $\delta \in \Delta\left(L_{K 3}\right)$ such that $\langle\delta, I(\delta)\rangle=0, p(\delta)=d$. Let $E=\phi^{-1}(\delta)$ be an effective divisor and $E=\sum m_{i} C_{i}+\sum n_{j} E_{j}$ be the irreducible decomposition where $C_{i}^{2} \geq 0, E_{j}^{2}=-2$ and $m_{i}, n_{j}>0$. By assumption, $\langle E, \iota(E)\rangle=0$. Thus, $0 \geq\left\langle\sum n_{j} E_{j}, \sum n_{j} \iota\left(E_{j}\right)\right\rangle$. Suppose that there is no $E_{j}$ such that $\left\langle E_{j}, \iota\left(E_{j}\right)\right\rangle=0$. Since there is no $\iota$-invariant -2 -curve and $\Gamma^{2} \geq-2$ for any irreducible curve, we get $\left\langle E_{j}, \iota\left(E_{j}\right)\right\rangle \geq 2$. Note that $\langle E, \iota(E)\rangle=(E+\iota(E))^{2} / 2-2 \equiv 0 \bmod 2$. Since

$$
\begin{aligned}
0 \geq\left\langle\sum n_{j} E_{j}, \sum n_{j} \iota\left(E_{j}\right)\right\rangle & \geq \sum_{E_{j}=\iota\left(E_{i}\right)}\left\langle n_{i} E_{i}+n_{j} E_{j}, n_{i} \iota\left(E_{i}\right)+n_{j} \iota\left(E_{j}\right)\right\rangle \\
& =\sum_{E_{j}=\iota\left(E_{i}\right)}\left\langle n_{i} E_{i}+n_{j} E_{j}, n_{i} E_{j}+n_{j} E_{i}\right\rangle \\
& \geq \sum_{E_{j}=\iota\left(E_{i}\right)} 2\left(n_{i}-n_{j}\right)^{2} \geq 0,
\end{aligned}
$$

we can rewrite $\sum n_{i} E_{i}=\sum n_{k}\left(E_{k}+\iota\left(E_{k}\right)\right)$. As $\left(E_{k}+\iota\left(E_{k}\right)\right)^{2} \geq 0$, it is impossible that $E^{2}=-2$. Thus, $\left\langle E_{j}, \iota\left(E_{j}\right\rangle=0\right.$ for some $E_{j}$ and we get the assertion. 
Lemma 10.12. $\Delta_{4,4}^{9}$ has an algebraic singularity along $H^{\prime}:=\mathcal{D}_{\mathrm{II}_{1,1}(2)}^{\prime} / \Gamma_{\mathrm{II}_{1,1}(2)}$.

Proof. Since special 2-elementary $K 3$ surfaces of type $\mathrm{II}_{1,1}(2)$ are realized as a double cover of singular quadric surface like special Enriques surfaces (cf. [B-P-V$\mathrm{V}$, Theorem 18.2]), we can prove the assertion analogously to Lemma 10.5.

Let us regard $\Delta_{4,4}^{9}$ as a $\Gamma_{\mathrm{II}_{1,1}(2)}$-invariant section of $\lambda_{\mathrm{II}_{1,1}(2)}^{\otimes 68}$ over $\Omega_{\mathrm{II}_{1,1}(2)}$.

Lemma 10.13. $\operatorname{div}\left(\Delta_{4,4}^{9}\right)=18 \mathcal{D}_{\mathrm{II}_{1,1}(2)}+l \mathcal{D}_{\mathrm{II}_{1,1}(2)}^{\prime}$ for some $l \in \mathbb{Z}$.

Proof. Since the projection map $\Omega_{\mathrm{II}_{1,1}(2)} \rightarrow \mathcal{M}_{\mathrm{II}_{1,1}(2)}$ ramifies of order 2 along the discriminant locus, we get the assertion by Lemmas 10.10, 10.11, and 10.12.

Let $e_{00}, e_{01}, e_{10}, e_{11}$ be the basis of $\mathbb{C}\left[T^{\vee} / T\right]=\mathbb{C}\left[\mathrm{II}_{1,1}(2)^{\vee} / \mathrm{II}_{1,1}(2)\right]$ such that $e_{\alpha \beta}$ corresponds to $(\alpha e+\beta f) / 2 \in \mathrm{II}_{1,1}(2)^{\vee}$. Let $f(\tau):=\eta(\tau)^{-8} \eta(2 \tau)^{-8}$ be a modular form for $\Gamma_{0}(2)$. Modifying Proposition 8.1 for $\Gamma_{0}(2)$, we can verify that

$\tilde{f}(\tau):=f(\tau) e_{00}+8\left\{f(\tau / 2)+f((\tau+1) / 2)\left(e_{00}+e_{01}+e_{10}\right)+8\left\{f(\tau / 2)-f((\tau+1) / 2) e_{11}\right.\right.$

is a modular form of type $\rho_{T}$ of weight -8 .

Proposition 10.4. $\Psi_{T}(z, f):=\Psi_{T}(z, \tilde{f})$ is a modular form of weight 68 with zero divisor $\mathcal{D}_{\mathrm{II}_{1,1}(2)}+16 \mathcal{D}_{\mathrm{II}_{1,1}(2)}^{\prime}$.

Proof. Since $\eta(\tau)^{-8} \eta(2 \tau)^{-8}=q^{-1}+8+O(q)$, we get

$$
\tilde{f}(\tau)=\left(q^{-1}+132+O(q)\right) e_{00}+O(1)\left(e_{01}+e_{10}\right)+\left(16 q^{-1 / 2}+O\left(q^{1 / 2}\right)\right) e_{11}
$$

which, together with Theorem 8.1, yields the assertion.

Theorem 10.2. $\Delta_{\mathrm{II}_{1,1}(2)}^{17}=C_{\mathrm{II}_{1,1}(2)} \Delta_{4,4}^{9}(z) / \Psi_{\mathrm{II}_{1,1}(2) \oplus \mathrm{II}_{1,17}}\left(z, \eta(\tau)^{-8} \eta(2 \tau)^{-8}\right)$.

Proof. Put $F(z):=\Delta_{4,4}^{9}(z) /\left[\Delta_{\mathrm{II}_{1,1}(2)}(z)^{17} \cdot \Psi_{\mathrm{II}_{1,1}(2) \oplus \mathrm{II}_{1,17}}\left(z, \eta(\tau)^{-8} \eta(2 \tau)^{-8}\right)\right]$. Since $\Delta_{\mathrm{II}_{1,1}(2)}$ is an automorphic form of weight $(-4,4), \Delta_{4,4}^{9}$ of $(0,68)$, and $\Psi_{T}(z, f)$ of $(68,0), F(z)$ is a $\Gamma_{\mathrm{II}_{1,1}(2)}$-invariant meromorphic function on $\Omega_{\mathrm{II}_{1,1}(2)}$. From Theorem 7.2, Lemma 10.3 and Proposition 10.4, it follows that $\operatorname{div}(F)=(l-$ $16) \mathcal{D}_{\mathrm{II}_{1,1}(2)}^{\prime}$. Thus, $F$ descends to a meromorphic function on $\mathcal{M}_{\mathrm{II}_{1,1}(2)}$ with divisor $(l-16) H^{\prime} / 2$. By the residue theorem, we get $l=16$ and $F$ must be a nonzero constant.

Remark. As in the case of Borcherds's $\Phi$-function, we can show

$$
\Psi_{\mathrm{II}_{1,1}(2) \oplus \mathrm{II}_{1,17}}\left(z, \eta(\tau)^{-8} \eta(2 \tau)^{-8}\right)^{2}=\Psi_{\mathrm{I}_{2,18}(2)}\left(z, \eta(\tau)^{-16} \eta(2 \tau)^{16} \eta(4 \tau)^{16}\right)
$$

if we regard $\mathrm{II}_{1,1}(2)=\left\{m h+n \delta \in \mathrm{I}_{1,1} ; m \equiv n \bmod 2\right\}$ and $\mathrm{II}(2) \oplus \mathrm{I}_{1,17}$ as a sublattice of $\mathrm{I}_{2,18}$. Here, $h^{2}=1, \delta^{2}=-1$, and $h \cdot \delta=0$ is the basis of $\mathrm{I}_{1,1}$. It is interesting that $\eta(\tau)^{-16} \eta(2 \tau)^{16} \eta(4 \tau)^{16}$ is the square of the modular form used to get Borcherds's $\Phi$-function. 


\section{§11. A Theta Product and Borcherds's Product}

The Nikulin type of the lattice $S_{5}$ in $(8.5)$ is $(r, l, \delta)=(16,6,1)$ and a 2 elementary $K 3$ surface of type $S_{5}$ is the minimal resolution of the double cover of $\mathbb{P}^{2}$ blanching along generic 6 lines. Therefore, the moduli space of 2-elementary $K 3$ surfaces of type $S_{5}$ is isomorphic to the configuration space $\mathbb{X}(3,6)$ of six points in $\mathbb{P}^{2}$, and the period map induces a morphism $\operatorname{Prd}: \mathbb{X}(3,6) \rightarrow \mathcal{M}_{S_{5}}$. In [Ma], Matsumoto described the inverse of the period map by using the theta function. Let $\mathbb{H}_{2}$ be the domain defined by $\mathbb{H}_{2}:=\left\{W \in M(2,2 ; \mathbb{C}) ;\left(W-W^{*}\right) / 2 i>0\right\}$ where $W^{*}={ }^{t} \bar{W}$. Identification of $\mathbb{H}_{2}$ and $\Omega_{S_{5}} \cong \Lambda_{5}+\sqrt{-1} C_{\Lambda_{5}}$ is given by

$$
\mathbb{H}_{2} \ni y=\left(\begin{array}{cc}
y_{0}+y_{1} & \frac{y_{0}+y_{1}+y_{2}+i y_{3}}{1+i} \\
\frac{y_{0}+y_{1}+y_{2}-i y_{3}}{1-i} & y_{0}+y_{2}
\end{array}\right) \rightarrow\left(1:-\operatorname{det} y: y_{0}: y_{1}: y_{2}: y_{3}\right) \in \Omega_{S_{5}}
$$

under which $\Gamma_{M}(1+i)$ (an arithmetic subgroup of $\left.\operatorname{Aut}\left(\mathbb{H}_{2}\right)\right)$ is identified with $\Gamma_{S_{5}}$ by [Ma, Proposition 1.5.1]. Note that the quadratic form attached to $\Lambda_{5}$ is given by $q(y)=4 \operatorname{det}(y)$. On $\mathbb{H}_{2}$ exist theta functions, ten of which are represented by

$$
\Theta_{a, b}(W)=\sum_{m \in \mathbb{Z}[\sqrt{-1}]^{2}} \exp \pi i\left\{(m+a)^{*} W(m+a)+2 \operatorname{Re} b^{*} m\right\}
$$

where $a, b \in\{0,(1+i) / 2\}^{2}$ with $a^{*} b \in \mathbb{Z}$. Any one of these ten theta functions is said to be an even theta function in this paper.

Theorem 11.1. Via the identification of $\Omega_{S_{5}}$ with $\mathbb{H}_{2}, 2^{12} \Delta_{S_{5}}=\prod_{\text {even }} \Theta_{a, b}$.

Proof. By [Ma, 1.4, Proposition 1.5.1 and Lemma 2.3.1], $\operatorname{div}\left(\Theta_{a, b}\right)$ is the $\Gamma_{S_{5}}$ orbit of $H_{\alpha(a, b)}+H_{\alpha^{\prime}(a, b)}$ where $\alpha(a, b), \alpha^{\prime}(a, b) \in \Delta\left(T_{5}\right)$ are roots defined in [Ma, 1.4]. (By [Ma, 2.3], $(a, b)$ corresponds uniquely to a partition of $\{1, \cdots, 6\}$ into $\{i, j, k\} \cup\{l, m, n\}$. Then, $\alpha(a, b):=\alpha(i, j, k)$ and $\alpha^{\prime}(a, b):=\alpha(l, m, n)$ in [Ma, 1.4].) In particular, on $\Omega_{S_{5}}$, one has

$$
\operatorname{div}\left(\prod \Theta_{a, b}\right)=\sum H_{\alpha(a, b)}+H_{\alpha^{\prime}(a, b)} .
$$

By [Ma, Proposition 3.1.1], $\left(\prod \Theta_{a, b}\right)^{2}$ becomes a modular form of weight 20 relative to $\Gamma_{M}(1+i)$. Consider the function $\Delta_{S_{5}}^{2} /\left(\prod \Theta_{a, b}\right)^{2}$ (or its higher power if necessary) which descents to a meromorphic function on $\mathcal{M}_{S_{5}}$ by the automorphic property. Compared with Theorem 7.2, it has no pole and thus is a constant. Namely, there exists a constant $C$ such that $C \Delta_{S_{5}}=\prod \Theta_{a, b}$. Comparing the first non-zero Fourier coefficient ([G-N1, (1.7)]), we get $C=2^{12}$.

$$
\text { Put } h=\left(\begin{array}{cc}
1 & \frac{1}{1+i} \\
\frac{1}{1-i} & 1
\end{array}\right), \delta_{6}=\left(\begin{array}{cc}
1 & \frac{1}{1+i} \\
\frac{1}{1-i} & 0
\end{array}\right), \delta_{7}=\left(\begin{array}{cc}
0 & \frac{1}{1+i} \\
\frac{1}{1-i} & 1
\end{array}\right), \delta_{8}=\left(\begin{array}{cc}
0 & \frac{i}{1+i} \\
\frac{-i}{1-i} & 0
\end{array}\right),
$$

and $\delta_{6}^{\prime}=h-\delta_{7}-\delta_{8}, \delta_{7}^{\prime}=h-\delta_{6}-\delta_{8}, \delta_{8}^{\prime}=h-\delta_{6}-\delta_{7}$. Then, the set of simple roots of $\Lambda_{5}$ is $\left\{\delta_{6}, \delta_{7}, \delta_{8}, \delta_{6}^{\prime}, \delta_{7}^{\prime}, \delta_{8}^{\prime}\right\}$, and the Weyl vector is $2 \rho_{5}=\sum_{6 \leq i \leq 8} \delta_{i}+\sum_{6 \leq i \leq 8} \delta_{i}^{\prime}$. Put $q_{i}=e^{2 \pi i y_{i}}(1=0,1,2,3), y=y_{0} h+y_{1} \delta_{6}+y_{2} \delta_{7}+y_{3} \delta_{8}$ and $r=r_{0} h+r_{1} \delta_{6}+$ $r_{2} \delta_{7}+r_{3} \delta_{8} \in \mathbb{H}_{2}$. Comparing Theorem 8.1 and 11.1, we get the following.

\section{Corollary 11.1.}

$2^{-12} \prod_{(a, b) \text { even }} \Theta_{a, b}(y)=q_{0}^{3} q_{1}^{-1} q_{2}^{-1} q_{3}^{-1} \prod_{\epsilon \in\{0,1\}} \prod_{r \in \Pi_{\epsilon}^{+}\left(\Lambda_{5}\right)}\left(1-q_{0}^{r_{0}} q_{1}^{-r_{1}} q_{2}^{-r_{2}} q_{3}^{-r_{3}}\right)^{c_{5}, \epsilon}(2 \operatorname{det} r)$

where $\Pi_{\epsilon}^{+}\left(\Lambda_{5}\right)=\left(\sum_{6 \leq i \leq 8}\left(\mathbb{Z}+\frac{\epsilon}{2}\right)_{\geq 0} \delta_{i}+\sum_{6 \leq i \leq 8}\left(\mathbb{Z}+\frac{\epsilon}{2}\right)_{\geq 0} \delta_{i}^{\prime}\right) \backslash\{0\}$, and $\left\{c_{5, \epsilon}(m)\right\}$ is the same as in Theorem 8.2. 


\section{REFERENCES}

[A]. Allcock, D., The period lattice for Enriques surfaces, math.AG/9905166 (1999).

[B-P-V-V]. Barth, W., Peters, C., Van de Ven, A., Compact Complex Surfaces, Springer.

[Be]. Beauville, A., Application aux éspace de module, Astérisque 126 (1985), 141-152.

[B-G-V]. Berline, N., Getzler, E., Vergne, M., Heat Kernels and Dirac Operators, Springer.

[B-C-O-V]. Bershadsky, M., Cecotti, S., Ooguri, H., Vafa, C., Kodaira-Spencer theory of gravity and exact results for quantum string amplitudes, Comm. Math. Phys. 165 (1994).

[Bi]. Bismut, J.-M., Equivariant immersions and Quillen metrics, J. Diff. Geom. 41 (1995), 53-157.

[B-B]. Bismut, J.-M., Bost, J.-B., Fibrés déterminants, métrique de Quillen et dégénérescence des courbes, Acta Math. 165 (1990), 1-103.

[B-G-S]. Bismut, J.-M., Gillet, H., Soulé, C., Analytic torsion and holomorphic determinant bundles I, II, III, Comm. Math. Phys. 115 (1988), 49-78, 79-126, 301-351.

[Bc]. Borcea, C., K3 surfaces with involution and mirror pairs of Calabi-Yau manifolds, "Essays on Mirror Manifolds II" (1996), International Press.

[Bo1]. Borcherds, R.E., Generalized Kac-Moody algebras, J. Alg. 115 (1988), 501-512.

[Bo2]. — Monstrous moonshine and monstrous Lie superalgebras, Invent. Math. 109 (1992), 405-444.

[Bo3]. - Automorphic forms on $O_{s+2,2}(\mathbb{R})$ and infinite products, Invent. Math. 120 (1995), 161-213.

[Bo4]. - The moduli space of Enriques surfaces and the fake monster Lie superalgebra, Topology 35 (1996), 699-710.

[Bo5]. - Automorphic forms with singularities on Grassmanians, Invent. Math. 132 (1998), 491-562.

[Bo6]. — , An automorphic form related to cubic surfaces, preprint.

[Bo7]. — private communications (e-mails to K.-I. Yoshikawa).

[B-K-P-S-B]. Borcherds, R., Katzarkov, L., Pantev, T., Shepherd-Barron, N., Families of K3 surfaces, J. Alg. Geom. 7 (1998), 183-193.

[B-C]. Bott, R., Chern, S.S., Hermitian vector bundles and the equidistribution of the zeros of their holomorphic sections, Acta Math. 114 (1968), 71-112.

[B-R]. Burns, D., Rapoport, M., On the Torelli problems for Kählerian K3 surfaces, Ann. Sci. Ec. Norm. Sup. IV Ser.8 (1975), 235-274.

[C-L]. Cheng, S.-Y., Li, P., Heat kernel estimates and lower bound of eigenvalues, Comment. Math. Helv. 56 (1981), 327-338.

[C-L-Y]. Cheng, S.-Y., Li, P., Yau, S.-T., On the upper estimate of the heat kernel of a complete Riemannian manifold, Amer. J. Math. 103 (1981), 1021-1063.

[C-S]. Conway, J.H., Sloane, N.J.A., Sphere Packings, Lattices and Groups, Springer.

[F]. Freitag, E., Siegelshe Modulfunktionen, Springer.

[G-T]. Gilberg, D., Trudinger, N.S., Elliptic Partial Differential Equations of Second Order, Springer.

[G-N1]. Gritsenko, V., Nikulin, V., Siegel automorphic form corrections of some Lorentzian Kac-Moody Lie algebras, Amer. J. Math. 119 (1997), 181-224.

[G-N2]. — K3 surfaces, Lorentzian Kac-Moody algebras and mirror symmetry, Math. Res. Lett. 3 (1996), 211-229.

[G-N3]. - Automorphic forms and Lorentzian Kac-Moody algebras, I, II, Intern. J. Math. 9 (1998), 153-199, 201-275.

[H-M]. Harvey, J., Moore, G., Exact gravitational threshold correction in the FHSV model, Phys. Rev. D 57 (1998), 2329-2336.

[J-K]. Jorgenson, J., Kramer, J., Star products of Green currents and automorphic forms, preprint (1998).

[J-T1]. Jorgenson, J., Todorov, A., A conjectured analogue of Dedekind's eta function for K3 surfaces, Math. Res. Lett. 2 (1995), 359-376.

[J-T2]. - Enriques surfaces, analytic discriminants, and Borcherds's $\Phi$-function, Comm. Math. Phys. 191 (1998), 249-264.

[J-T3]. — A correction for the paper "Enriques surfaces ...", preprint (1998).

[K-R]. Köhler, K., Roessler, D., A fixed point formula of Lefschetz type in Alakelov geometry I,II,III, preprint $(1998,1999)$. 
[Kt]. Katz, N.M., Pinceaux de Lefschetz: théorème d'existence, SGA 7,II, Lect. Notes Math. 340 (1973), 213-253.

[Kz]. Kazhdan, D., Connection of the dual space of a group and the structure of its closed subgroups, Funct. Anal. Appl. 1 (1967), 63-65.

[Ko]. Kobayashi, R., Moduli of Einstein metrics on a K3 surface and degeneration of type I, Adv. Study Pure Math. 18-II (1990), 257-311.

[K-T]. Kobayashi, R., Todorov, A., Polarized period map for generalized K3 surfaces and the moduli of Einstein metrics, Tohoku Math. J. 39 (1987), 341-363.

[Kn]. Kondō, S., The rationality of the moduli space of Enriques surfaces, Compositio Math. 91 (1994), 159-173.

[Kr]. Kronheimer, P.B., The construction of ALE spaces as hyper-Kähler quotients, J. Diff. Geom. 29 (1989), 665-683.

[L-Y]. Li, P., Yau, S.-T., On the parabolic kernel of the Schrödinger operator, Acta Math. 156 (1986), 153-201.

[Ma]. Matsumoto, K., Theta function on the bounded symmetric domain of type $\mathrm{I}_{2,2}$ and the period map of a 4-parameter family of K3 surfaces, Math. Ann. 295 (1993), 383-409.

[Man]. Manin, Y., Cubic Forms, North Holland.

[Mo]. Morrison, D., Some remarks on the moduli of K3 surfaces, Progress in Math. 39 (1983), 303-332.

[Na]. Namikawa, Y., Periods of Enriques surfaces, Math. Ann. 270 (1985), 201-222.

[Ni1]. Nikulin, V.V., Integral symmetric bilinear forms and some of their applications, Math. USSR Izvestija 14 (1980), 103-167.

[Ni2]. Nikulin, V.V., Finite automorphism groups of Kähler K3 surfaces, Trans. Moscow Math. Soc. 38 (1980), 71-135.

[Ni3]. - Involution of integral quadratic forms and their applications to real algebraic geometry, Math. USSR Izvestiya 22 (1984), 99-172.

[Ni4]. - On the quotient groups of the automorphism group of hyperbolic forms by the subgroup generated by 2-reflections, J. Soviet Math. 22 (1983), 1401-1476.

[P-S-S]. Piatetskii-Shapiro, I., Shafarevich, I.R., A Torelli theorem for algebraic surfaces of type K3, Math. USSR Izv. 35 (1971), 530-572.

[R-S]. Ray, D.B., Singer, I.M., Analytic torsion for complex manifolds, Ann. of Math. 98 (1973), 154-177.

[Sc]. Schumacher, G., On the geometry of moduli spaces, Manuscripta Math. 50 (1985), 229-267.

[Sha]. Shah, J., A complete moduli space for K3 surface of degree 2, Ann. of Math. 112 (1980), 485-510.

[Si]. Siu, U.-T., Lectures on Hermitian-Einstein Metrics for Stable Bundles and KählerEinstein Metrics, Birkhäuser.

[Ti]. Tian, G., Smoothness of the universal deformation space of compact Calabi-Yau manifolds and its Petersson-Weil metric, "Mathematical Aspects of String Theory, 1987", Adv. Studies in Math. Physics 1 (1987).

[To1]. Todorov, A., Applications of the Kähler-Einstein-Calabi-Yau metric to moduli of K3 surfaces, Invent. Math. 81 (1980), 251-266.

[To2]. — The Weil-Pertersson geometry of the moduli space of $S U(n \geq 3)$ (CalabiYau) manifolds I, Comm. Math. Phys. 126 (1989), 325-346.

[V]. Voisin, C., Miroirs et involutions sur les surfaces K3, Asterisque 218 (1993), 273322.

[Ya]. Yau, S.-T., On the Ricci curvature of a compact Kähler manifold and the complex Monge-Ampère Equation, I, Comm. Pure Appl. Math. 31 (1978), 339-411.

[Yo1]. Yoshikawa, K.-I., Degeneration of algebraic manifolds and the spectrum of Laplacian, Nagoya Math. J. 146 (1997), 83-129.

[Yo2]. — Discriminant of theta divisors and Quillen metrics, math.AG/9904092 (to appear in J. Diff. Geom.) (1999).

Graduate School of Mathematics, Nagoya University, Nagoya 464-8602, Japan

E-mail address: yosikawa@math.nagoya-u.ac.jp 